\title{
Multi-dimensional Diffusion Waves for the Navier-Stokes Equations of Compressible Flow
}

\author{
David Hoff \& Kevin Zumbrun
}

Abstract. We derive a detailed, pointwise description of the asymptotic behavior of solutions of the Cauchy problem for the Navier-Stokes equations of compressible flow in several space dimensions, with initial data in $L^{1} \cap H^{k(n)}$. We show that, asymptotically, the solution decomposes into the sum of two terms, one of which dominates in $L^{p}$ for $p>2$, the other for $p<2$. The dominant term for $p>2$ has constant density and divergence-free momentum field, decaying at the rate of a heat kernel. Thus, as measured in $L^{p}$ for $p>2$, all smooth, small-amplitude solutions of the Navier-Stokes equations are asymptotically incompressible. When $p<2$, the dominant term reflects instead the spreading effect of convection, and decays more slowly than a heat kernel; in fact, the solution may grow without bound in $L^{p}$ for $p$ near 1 . These features of the solution do not arise in one dimensional flow, nor are they apparent from previously known $L^{2}$ decay rates. An alternative interpretation of our estimates shows that the solution is asymptotically given by a diffusion about the origin whose mass is that of the initial data, convected by the fundamental solution of the linearized Euler equations. This result thus defines the correct notion of "diffusion wave" in the context of compressible, Navier-Stokes flows. Our analysis involves a number of interesting issues in Fourier multiplier and PaleyWiener theory, required for the derivation of pointwise bounds from representations of the Fourier transforms of Green's matrices. 
1. Introduction. In this paper we derive a detailed description of the asymptotic behavior of solutions of the Cauchy problem for the Navier-Stokes equations of compressible flow in several space variables,

$$
\left\{\begin{array}{l}
\rho_{t}+\operatorname{div} m=0 \\
m_{t}^{j}+\operatorname{div}\left(\frac{m^{j} m}{\rho}\right)+P(\rho)_{x_{j}}=\varepsilon \Delta\left(\frac{m^{j}}{\rho}\right)+\eta \operatorname{div}\left(\frac{m}{\rho}\right)_{x_{j}}
\end{array}\right.
$$

with smooth initial data $\left[\begin{array}{c}\rho_{0} \\ m_{0}\end{array}\right]$ close to a constant state $\left[\begin{array}{c}\rho^{*} \\ m^{*}\end{array}\right]$, which without loss of generality we take to be $\left[\begin{array}{l}1 \\ 0\end{array}\right]$. Here $t \geq 0$ is time, $x \in \mathbb{R}^{n}$ is the spatial coordinate, and $\rho(x, t), m(x, t)=\left(m^{1}(x, t), \ldots, m^{n}(x, t)\right)$, and $P=P(\rho)$ represent the fluid density, momentum density, and pressure; (thus $m / \rho$ is the particle velocity). $\varepsilon>0$ and $\eta \geq 0$ are viscosity constants, and div and $\Delta$ are the usual spatial divergence and Laplace operators.

We derive explicit pointwise bounds for the asymptotic state of the perturbation

$$
u(x, t)=\left[\begin{array}{c}
\rho(x, t) \\
m(x, t)
\end{array}\right]-\left[\begin{array}{c}
\rho^{*} \\
m^{*}
\end{array}\right]
$$

in terms of $x$ and $t$; these bounds then yield decay estimates for $D_{x}^{\alpha} u(\cdot, t)$ in $L^{p}\left(\mathbb{R}^{n}\right)$ for all $p \in[1, \infty]$. We find that $\left[\begin{array}{c}\rho(\cdot, t) \\ m(\cdot, t)\end{array}\right]$ is time-asymptotic to the solution $\left[\begin{array}{c}\tilde{\rho}(\cdot, t) \\ \tilde{m}(\cdot, t)\end{array}\right]$ of the following linear effective artificial viscosity system, which is associated with the nonlinear Navier-Stokes equations (1.1) in a canonical way:

$$
\left\{\begin{array}{l}
\tilde{\rho}_{t}+\operatorname{div} \tilde{m}=\frac{1}{2}(\varepsilon+\eta) \Delta \tilde{\rho} \\
\tilde{m}_{t}+c^{2} \nabla \tilde{\rho}=\varepsilon \Delta \tilde{m}+\frac{1}{2}(\eta-\varepsilon) \nabla \operatorname{div} \tilde{m},
\end{array}\right.
$$

where $c^{2}=P^{\prime}\left(\rho^{*}\right)=P^{\prime}(1)$. The system (1.3) is uniquely characterized by the approximation property above, by its strict parabolicity, and by the fact that the symbol of its first-order convection terms commutes with that of its second-order diffusion terms. These properties enable us to give a very detailed description of the asymptotic behavior of $\left[\begin{array}{c}\tilde{\rho}(\cdot, t) \\ \tilde{m}(\cdot, t)\end{array}\right]$, and thereby that of $\left[\begin{array}{c}\rho(\cdot, t) \\ m(\cdot, t)\end{array}\right]$. We find that, modulo the constant state $\left[\begin{array}{c}\rho^{*} \\ m^{*}\end{array}\right]$, the solution $\left[\begin{array}{c}\rho(\cdot, t) \\ m(\cdot, t)\end{array}\right]$ of $(1.1)$ is 
asymptotically the sum of two terms, one of which dominates in $L^{p}$ for $p>2$, the other for $p<2$. For $p>2$ the dominant term has constant density $\rho^{*}$ and a nonconstant, divergence-free momentum field, decaying at the rate of a heat kernel, which is the rate of decay of the solution of a linear, second order, strictly parabolic system with $L^{1}$ initial data. This result gives the very important conclusion that, as measured in $L^{p}\left(\mathbb{R}^{n}\right)$ for $p>2$, all smooth, smallamplitude solutions of the nonlinear Navier-Stokes equations (1.1) are asymptotically incompressible. Thus, overall, the perturbation $u(\cdot, t)$ defined in (1.2) decays at the heat kernel rate, but the difference between the solution $\left[\begin{array}{c}\rho(\cdot, t) \\ m(\cdot, t)\end{array}\right]$ and the aforementioned incompressible flow decays more rapidly, at a rate given explicitly in terms of $n$ and $p$. For $p<2$ the situation is quite different. Here the asymptotically dominant term reflects the spreading effect of the solution operator for the standard multi-dimensional wave equation. As a result, the perturbation $u(\cdot, t)$ may actually grow without bound in $L^{p}\left(\mathbb{R}^{n}\right)$ for $p<2$; for example, $\|u(\cdot, t)\|_{L^{1}} \sim t^{1 / 2}$ when $n=3$. Both these effects, the decay to an incompressible flow in $L^{p}$ for $p>2$, and the retardation of the parabolic decay in $L^{p}$ for $p<2$, are truly multidimensional in character: the first is without significance in one space dimension, and the second does not occur.

Our work extends that of Zeng [22], Liu and Zeng [16], and Kawashima [8]-[9]. Zeng and Liu study general hyperbolic-parabolic systems in one space variable. Just as here, they obtain $L^{p}$ decay rates for $p \in[1, \infty]$ by integrating pointwise bounds. They find that, for problems in one space dimension, the perturbation decays at the heat kernel rate in all $L^{p}$ norms. Kawashima [8] studies general hyperbolic-parabolic systems in several space variables, deriving decay rates in $H^{s}\left(\mathbb{R}^{n}\right)$ by Fourier transform techniques. $L^{p}$ estimates are then obtained by interpolation, but for $p \geq 2$ only. Just as here, he finds that the overall decay rate in these norms is the heat kernel rate, but he does not obtain the incompressible limit result alluded to above, which is special to the Navier-Stokes system, nor can his techniques address the question of pointwise estimates and $L^{p}$ behavior for $p<2$. Still, the initial part of our analysis parallels that of Kawashima, and we make important use of his idea of an effective artifical viscosity system. To obtain explicit pointwise bounds for the asymptotic state in terms of $x$ and $t$, however, we must go considerably beyond the usual analysis based on the Hausdorff-Young inequality; this leads us to address several interesting questions in harmonic analysis related to Fourier multiplier and Paley-Wiener theory. As a by-product of this analysis we are able to reduce significantly the smoothness requirements on the initial data.

Because the artificial viscosity system (1.3) is linear and strictly parabolic, standard techniques enable us to show that, when the initial perturbation $u_{0}=$ $\left[\begin{array}{c}\rho_{0} \\ m_{0}\end{array}\right]-\left[\begin{array}{c}\rho^{*} \\ m^{*}\end{array}\right]$ has a first moment, the corresponding solution $\left[\begin{array}{c}\tilde{\rho} \\ \tilde{m}\end{array}\right]-\left[\begin{array}{c}\rho^{*} \\ m^{*}\end{array}\right]$ of 
(1.3) is asymptotic to the Green's matrix $\tilde{G}$ for (1.3), multiplied by the total mass vector $U$ of $u_{0}$. Our results therefore show that the same will be true also for the solution $u=\left[\begin{array}{c}\rho \\ m\end{array}\right]-\left[\begin{array}{c}\rho^{*} \\ m^{*}\end{array}\right]$ of the nonlinear Navier-Stokes system (1.1). This asymptotic flow $\tilde{G} U$ has its mass initially concentrated at the origin, and, owing to the special properties of $\tilde{G}$ discussed above, can be interpreted as a simple diffusion convected by the fundamental solution of the linearized Euler equations. $\tilde{G} U$ is therefore a "diffusion wave," and an important corollary of our results is therefore that the perturbation $u$ associated with the solution of the nonlinear Navier-Stokes equations (1.1) is asymptotic to a diffusion wave whose total mass is that of the initial perturbation.

The importance of diffusion waves for problems in one space dimension was first recognized by Liu [12]: diffusion waves decay in $L^{p}$ for all $p>1$, but not in $L^{1}$. These waves therefore "carry mass," and so are critical for describing the asymptotic behavior of solutions of hyperbolic-parabolic systems when the initial data has differing states at $x=+\infty$ and $x=-\infty$. In particular, onedimensional diffusion waves have played an essential role in the study of the stability properties of viscous shocks, rarefactions, and contact discontintuities. See for example Liu [12]-[13], Szepessy and Xin [20], Szepessy and Zumbrun [21], Liu and Zumbrun [14], and Liu and Xin [15]. All these analyses require in an essential way an explicit, pointwise description of the one-dimensional diffusion wave. The importance of the present work is therefore that, by identifying explicitly the multidimensional diffusion wave, and by studying its pointwise properties, we prepare the way for the analysis of the large-time behavior of solutions of (1.1) when the initial data is not constant at $|x|=\infty$, and the asymptotic flow includes nontrivial, nonlinear waves.

We now give a more precise formulation of our results. First, we shall assume throughout that the pressure $P$ is smooth in a neighborhood of $\rho^{*}$, with $c^{2}=P^{\prime}\left(\rho^{*}\right)>0$. Next we linearize the system (1.1) about the constant state $\left[\begin{array}{c}\rho^{*} \\ m^{*}\end{array}\right]=\left[\begin{array}{l}1 \\ 0\end{array}\right]$, to obtain the approximate system

$$
\left\{\begin{array}{l}
\rho_{t}+\operatorname{div} m=0 \\
m_{t}+c^{2} \nabla \rho=\varepsilon \Delta m+\eta \nabla \operatorname{div} m .
\end{array}\right.
$$

We shall make important use of the respective Green's matrices $G$ and $\tilde{G}$ for the linear systems (1.4) and (1.3): the entries of $G$ and $\tilde{G}$ map the time axis $[0, \infty)$ into the set of tempered distributions $\mathcal{S}^{\prime}\left(\mathbb{R}^{n}\right)$; the columns of $G$ and $\tilde{G}$ satisfy the respective systems (1.4) and (1.3); and $G(0)=\tilde{G}(0)=\delta I$, where $\delta$ is the usual Dirac distribution and $I$ is the identity matrix. The solutions of (1.4) 
and (1.3) with initial data $u_{0}$ are therefore the spatial convolutions $G * u_{0}$ and $\tilde{G} * u_{0}$.

Our analysis of the large-time behavior of the perturbation $u(\cdot, t)$ defined in (1.2) then proceeds in a sequence of successively more refined approximations. First we show that $u(\cdot, t)$ is time-asymptotic to $G * u_{0}$ in the sense that the difference, which is the linearization error, decays more rapidly than does $u(\cdot, t)$. Next, because of the special relationship between the linearized system (1.4) and the effective artificial viscosity system (1.3), we are able to show that the difference $G(t)-\tilde{G}(t)$ between their Green's matrices also decays more rapidly than does $u(\cdot, t)$. Thus $u(\cdot, t)$ is equally well-approximated by $\tilde{G} * u_{0}$ for large time. The strict parabolicity of the system (1.3), together with the commutativity of symbols property mentioned above, enables us to derive explicit, pointwise estimates for $\tilde{G}(t)$, and therefore for the perturbation $u(\cdot, t)$, by way of the approximations above. Rates of growth and decay for $\left\|D_{x}^{\alpha} u(\cdot, t)\right\|_{L^{p}}$ are then obtained by integration of these pointwise bounds, and our incompressible limit result follows from a very detailed study of the structure of $\tilde{G}$. Finally, we connect our results with the one-dimensional theory in a somewhat different way by showing that $\left[\begin{array}{c}\rho \\ m\end{array}\right]$ is also well approximated for large time by the solution of the following nonlinear artificial viscosity system:

$$
\left\{\begin{array}{l}
\rho_{t}+\operatorname{div} m=\frac{1}{2}(\varepsilon+\eta) \Delta \rho, \\
m_{t}+\left(\frac{m^{k} m}{\rho}\right)_{x_{k}}+\nabla P(\rho)=\varepsilon \Delta m+\frac{1}{2}(\eta-\varepsilon) \nabla \operatorname{div} m .
\end{array}\right.
$$

The system (1.5) results by retaining the nonlinear convection terms in the Navier-Stokes system (1.1), but replacing its nonlinear, nondefinite diffusion terms with the strictly parabolic, linear diffusion terms of (1.3). The system (1.5) is therefore the multidimensional analogue of those approximating systems for one-dimensional problems obtained by lifting solutions of the viscous Burgers equation to the various characteristic fields.

The following theorem contains the principal results of this paper. We overstate somewhat the regularity hypotheses on $u_{0}$ in order to avoid technicalities and to highlight the essential features. Complete statements, with less severe regularity conditions, are given in Theorems 5.3, 6.3, 6.7, 6.8, and 8.2. We remind the reader that, if $K_{\vartheta}$ is the standard heat kernel, that is, the solution of the scalar heat equation $\left(K_{\vartheta}\right)_{t}=\vartheta \Delta K_{\vartheta}$ with initial data $K_{\vartheta}(\cdot, 0)=\delta$, then

$$
\left\|D_{x}^{\alpha} K_{\vartheta}(\cdot, t)\right\|_{L^{p}\left(\mathbb{R}^{n}\right)} \leq C(\alpha, \vartheta) t^{n / 2(1 / p-1)-|\alpha| / 2}, p \in[1, \infty] .
$$

We therefore define $r_{\alpha, p}=(n / 2)(1-1 / p)+|\alpha| / 2$, and suggest that the various rates of decay in the following statement be compared with the rates of decay for $K_{\vartheta}$ above. 
Theorem. Let $P,\left[\begin{array}{c}\rho^{*} \\ m^{*}\end{array}\right]$, and $u_{0}=\left[\begin{array}{c}\rho_{0} \\ m_{0}\end{array}\right]-\left[\begin{array}{c}\rho^{*} \\ m^{*}\end{array}\right]$ be as above, with

$$
E=\left\|u_{0}\right\|_{L^{1}}+\left\|u_{0}\right\|_{H^{[n / 2]+l}}
$$

sufficiently small, where $\ell \geq 3$ is an integer. Then the Navier-Stokes system (1.1) with initial data $\left[\begin{array}{c}\rho_{0} \\ m_{0}\end{array}\right]$ has a global solution satisfying the following decay estimates for all multi-indices $\alpha$ with $|\alpha| \leq(\ell-3) / 2$ and any positive $\sigma$ :

$$
\left\|D_{x}^{\alpha} u(\cdot, t)\right\|_{L^{p}} \leq C(\ell) E \begin{cases}(1+t)^{-r_{\alpha, p}}, & 2 \leq p \leq \infty \\ (1+t)^{-r_{\alpha, p}+((n-1) / 4)(2 / p-1)} L_{\alpha}(t), & 1 \leq p<2\end{cases}
$$

where $L_{\alpha}(t)=\log (1+t)$ when $n=2$ and $\alpha=0$, and is otherwise identically one;

$$
\begin{array}{r}
\left\|D_{x}^{\alpha}\left(u-G * u_{0}\right)(\cdot, t)\right\|_{L^{p}},\left\|D_{x}^{\alpha}\left(u-\tilde{G} * u_{0}\right)(\cdot, t)\right\|_{L^{p}},\left\|D_{x}^{\alpha}(u-\tilde{u})(\cdot, t)\right\|_{L^{p}} \\
\leq E L(t) \begin{cases}C(\ell)(1+t)^{-r_{\alpha, p}-1 / 2}, & 2 \leq p \leq \infty \\
C(\ell, \sigma)(1+t)^{-r_{\alpha, p}+(n / 4)(2 / p-1)-1 / 2+\sigma}, & 1 \leq p<2,\end{cases}
\end{array}
$$

where $L(t)=\log (1+t)$ when $n=2$ and is otherwise identically one;

$$
\begin{aligned}
& \left\|D_{x}^{\alpha}\left(\left[\begin{array}{c}
\rho(\cdot, t) \\
m(\cdot, t)
\end{array}\right]-\left[\begin{array}{c}
\rho^{*} \\
K_{\varepsilon} * m_{0 f}
\end{array}\right]\right)\right\|_{L^{p}} \\
\leq & C(\ell) E(1+t)^{-r_{\alpha, p}+((n-1) / 4)(2 / p-1)}, \quad p \in[2, \infty],
\end{aligned}
$$

where $m_{0 f}$ is the divergence-free part of $m_{0}$ (see (1.12) below); and finally if $\int|x|\left|u_{0}(x)\right| d x \equiv \mu$ is finite, then

$$
\begin{aligned}
& \left\|D_{x}^{\alpha}(u-\tilde{G} U)(\cdot, t)\right\|_{L^{p}} \\
\leq & (E+\mu) \begin{cases}C(|\alpha|)(1+t)^{-r_{\alpha, p}-1 / 2}, & 2 \leq p \leq \infty, \\
C(|\alpha|, \sigma)(1+t)^{-r_{\alpha, p}+(n / 4)(2 / p-1)-1 / 2+\sigma}, & 1 \leq p<2,\end{cases}
\end{aligned}
$$

where $U \equiv \int u_{0}(x) d x$.

(1.6) therefore shows that the perturbation $u(\cdot, t)$ decays at the rate of a heat kernel in $L^{p}$ for $p \geq 2$, but less rapidly for $p<2$; in fact, the bound in (1.6) for $u(\cdot, t)$ may actually grow with increasing $t$. (1.6) and (1.7) then show that $u(\cdot, t)$ is time-asymptotic to each of $G(t) * u_{0}, \tilde{G}(t) * u_{0}$, and $\tilde{u}(\cdot, t)$, in the sense 
that the respective differences are negligible compared to the estimated size of $u(\cdot, t)$.

We shall discuss (1.8) and (1.9) in more detail, in order to highlight some of the important aspects of our analysis which do not appear explicitly in the statements above. First, we let $G^{B}$ be the Green's matrix for the strictly parabolic system

$$
\left\{\begin{array}{l}
\rho_{t}=\frac{1}{2}(\varepsilon+\eta) \Delta \rho \\
m_{t}=\varepsilon \Delta m+\frac{1}{2}(\eta-\varepsilon) \nabla \operatorname{div} m,
\end{array}\right.
$$

and $W$ the Green's matrix for the hyperbolic system

$$
\left\{\begin{array}{l}
\rho_{t}+\operatorname{div} m=0 \\
m_{t}+c^{2} \nabla \rho=0 .
\end{array}\right.
$$

As we shall see, $G^{B}$ and $W$ commute, so that the Green's matrix $\tilde{G}$ for the artificial viscosity system (1.3) is simply the convolution $\tilde{G}=G^{B} * W$. Now, because the system (1.10) is strictly parabolic, $G^{B}$ will have all the decay properties of an ordinary heat kernel. The hyperbolic system (1.11), on the other hand, which consists of the linearized Euler equations, is somewhat more complex. First observe that (1.11) has nontrivial steady-state solutions, namely, those flows $\left[\begin{array}{c}\rho \\ m\end{array}\right]$ for which $\rho$ is constant and $\operatorname{div} m=0$; these are time-independent, incompressible flows. This leads us to consider the projection $\Pi$ defined by

$$
\Pi *\left[\begin{array}{c}
\rho_{0} \\
m_{0}
\end{array}\right]=\left[\begin{array}{c}
0 \\
m_{0 f}
\end{array}\right],
$$

where $m_{0 f}$ is the divergence-free part of $m_{0}$,

$$
\widehat{m}_{0 f}(\xi)=\left(I-\frac{\xi \xi^{t}}{|\xi|^{2}}\right) \widehat{m}_{0}(\xi) \text {. }
$$

We then write $W_{f}=W * \Pi$ and $W=W_{f}+W_{\perp}$. Then since $W_{f}$ has nontrivial fixed points, $W_{f} * u_{0}$ cannot in general decay. On the other hand, we find that $W_{\perp}$ has precisely the growth and decay characteristics of the fundamental solution for the standard wave equation. These observations lead us to decompose the perturbation $u(\cdot, t)$ as follows:

$$
\begin{aligned}
u & =\tilde{G} * u_{0}+(G-\tilde{G}) * u_{0}+\left(u-G * u_{0}\right) \\
& =G^{B} * W_{f} * u_{0}+G^{B} * W_{\perp} * u_{0}+(G-\tilde{G}) * u_{0}+\left(u-G * u_{0}\right) .
\end{aligned}
$$


Now, (1.7) shows that the last two terms on the right here are negligible for large time. Estimating the convolutions in the first two terms, we find that the first decays at precisely the heat kernel rate in all $L^{p}$, which is exactly what is expected, since $W_{f}$ has nontrivial fixed points and (1.10) is strictly parabolic; and the second term $G^{B} * W_{\perp} * u_{0}$ decays at the heat kernel rate times the factor $(1+t)^{((n-1) / 4)(2 / p-1)}$. This latter factor is neutral when $p=2$, enhances the decay rate for $p>2$, and retards it for $p<2$. Thus it is the diffusing, incompressible flow $G^{B} * W_{f} * u_{0}$ which dominates in $L^{p}$ for $p>2$; expressing this result in terms of $\rho$ and $m$, we thus obtain statement (1.8) of the theorem. When $p<2$, the situation reverses itself, and it is the second term in (1.13) which dominates, and which accounts for the overall rate of decay of $u(\cdot, t)$ given in (1.6).

The statement in (1.9) gives an alternative interpretation of our asymptotic description of $u(\cdot, t)$. Applying the above factorization $\tilde{G}=W * G^{B}$ to the result (1.9), we find that $u(\cdot, t)$ is time-asymptotic to the flow $W *\left(G^{B} U\right)$, where $U$ is the total mass of $u_{0}$. Now, since $G^{B}$ is the fundamental solution of a strictly parabolic system, $G^{B} U$ may be regarded as a diffusion about the origin, with total mass $U$. The asymptotic state $u \sim W *\left(G^{B} U\right)$ is therefore the diffusion from a single point, convected by the fundamental solution $W$ of the linearized Euler equations; that is, $u(\cdot, t)$ is asymptotically a "diffusion wave."

The two points of view expressed in (1.8) and (1.9) are reflected in the following pointwise bounds for $\tilde{G}$, which are derived from a detailed analysis of the convolutions $\tilde{G}=G^{B} * W_{f}+G^{B} * W_{\perp}$ : we find that, when $n=3$,

$$
\left|\left(\tilde{G}^{B} * W_{f}\right)(x, t)\right| \leq C \min \left\{t^{-3 / 2},|x|^{-3}\right\}
$$

and

$$
\left|\left(\tilde{G}^{B} * W_{\perp}\right)(x, t)\right| \leq C t^{-2} \exp \left(-\frac{(|x|-c t)^{2}}{C t}\right) .
$$

There is no hyperbolic effect in the bound for $G^{B} * W_{f}$ above since, as we saw above, the operator $W_{f} *$ has nontrivial fixed points. On the other hand, the bound for $G^{B} * W_{\perp}$ reflects the interaction of the contractive hyperbolic operator $W_{\perp}$ with the three-dimensional diffusion $G^{B} \sim t^{-3 / 2} e^{-|x|^{2} / C t}$ : the bulk of the mass of $G^{B}$ is convected along the light cone $\{x:|x|=c t\}$, and its pointwise decay rate is increased by the factor $t^{-1 / 2}$, which is precisely the term $t^{((n-1) / 4)(2 / p-1)}$ with $n=3$ and $p=\infty$.

We remark that, in one space dimension, the system (1.1) can be simplified by the introduction of Lagrangean coordinates $(v, q)$, where $v$ is the specific volume and $q$ is the velocity. By working in these coordinates and applying the arguments of Theorems 5.3 and 8.3, together with the energy estimates of 
Hoff [2], we can extend our estimates to the case of fairly rough initial data $v_{0}-v^{*} \in L^{\infty} \cap L^{1}$ and $q_{0}-q^{*} \in L^{1} \cap L^{2}$. A corresponding existence and regularity theory for solutions of (1.1) in two and three space dimensions has been developed in Hoff [3] and [4] and in Lions [11], but it remains an open question whether the smoothness requirements of the present theorem can be relaxed for the multidimensional case.

To provide a context for the results above, we give a survey of some related work, and a brief history of some of the ideas related to those of the present paper. Aside from its importance in applications, the Navier-Stokes system (1.1) is of particular interest mathematically because of its hyperbolic-parabolic character: there is no smoothing in the mass equation, and in most applications the dominant physical forces are those represented by the hyperbolic terms. One of the most important aspects of this system is therefore the interaction between convective and diffusive effects. As we shall see, this interaction results in a weak smoothing of the density, and this fact turns out to be crucial in nearly all the known results.

A precise sense for this observation has evolved gradually, through a series of interesting and related results. The first of these was Kan'el's proof [7] of global existence of solutions of (1.1) in one space dimension, wherein the compensating effect of the hyperbolic terms is revealed indirectly, via nonstandard energy estimates augmenting the usual entropy-type bounds. The result is that both the density $\rho$ and the velocity $q$ are controlled in all derivatives, but smoothing of the initial data is apparent only in the $q$-coordinates. These ideas were then extended to two and three space dimensions by Matsumura and Nishida [17].

Later, Hoff [2] proved global existence of weak solutions of (1.1), in one space dimension, in the case of rough initial data. His analysis in the setting of weak solutions clarifies the nature of the smoothing of the density $\rho$ : discontinuities in $\rho$ are shown to persist for all time, convecting along particle trajectories; however, their strengths decay exponentially in time, more rapidly for smaller viscosities. This exponential decay is crucial for the analysis, and is shown to be a consequence of the hyperbolic character of the corresponding inviscid equations; thus these interesting, new phenomena are shown to result precisely from the interaction of hyperbolic and parabolic effects.

Kawashima was the first to study rates of decay and specific asymptotic behavior of solutions of (1.1), in the process offering further insight into the compensative mechanism of hyperbolic effects. In [8], he identified a general class of "stable" hyperbolic-parabolic systems exhibiting such compensation, characterized primarily by the requirement that the associated constant coefficient system,

$$
u_{t}+A_{j} u_{x_{j}}+B_{j k} u_{x_{j} x_{k}}=0,
$$


obtained by linearizing around a constant state, be asymptotically stable in $L^{2}$. By studying the spectral properties of the Fourier transform of the corresponding Green's matrix

$$
\widehat{G}(\xi, t)=e^{\left(-i \xi_{j} A_{j}-\xi_{j} \xi_{k} B_{j k}\right) t},
$$

Kawashima proved that $L^{2}$-linearized stability is equivalent to the condition that

$$
|\widehat{G}(\xi, t)| \leq C_{1} e^{-\mu|\xi|^{2} t}+C_{2} e^{-\gamma t},
$$

for constants $\gamma, \mu>0$. The estimate (1.14) is obtained by perturbation expansions around $|\xi|=0$ and $|\xi|=\infty$, and results in the decay rates

$$
\left\|D_{x}^{\alpha}\left(G(\cdot, t) * u_{0}\right)\right\|_{2} \leq C_{1} t^{-n / 4-|\alpha| / 2}\left\|u_{0}\right\|_{1}+C_{2} e^{-\gamma t}\left\|D_{x}^{\alpha} u_{0}\right\|_{2} .
$$

Estimates for solutions of the original nonlinear system then follow by an iteration argument. Kawashima also determined the asymptotic behavior of solutions in one space dimension and for $p \geq 2$, by applying similar techniques. Observe that we see here in (1.14) the same phenomenon of exponential decay in time that was observed for the one-dimensional case in [2].

The Fourier methods of Kawashima suffice to give information about asymptotic behavior in $L^{2}$, and then via Sobolev bounds, in $L^{p}$ for $p \geq 2$. As for decay in lower $L^{p}$ spaces, Zeng [22] has given an analysis which completes the picture for problems in one space dimension by proving decay estimates for $p \in[1,2]$. Her analysis involves the decomposition of the Green's matrix $G$ into the sum

$$
G(x, t)=e^{-b t}\left[\begin{array}{ll}
1 & 0 \\
0 & 0
\end{array}\right] \delta\left(x-q^{*} t\right)+G_{1}(x, t),
$$

where the first term is a $\delta$-function decaying exponentially in time, and $G_{1} \in L^{1}$ is a matrix function for which sharp, pointwise bounds can be obtained. The explicit description (1.15) of the Green's function unifies the various perspectives above, and gives a precise sense to the nature of hyperbolic compensation. In particular, we now see an explicit connection with the results of Hoff [2]: the solution operator supports discontinuties in the $\rho$-coordinate, convected along particle paths at the background speed $q^{*}$, and decaying as $e^{-b t}$. These results have recently been extended to general hyperbolic-parabolic systems in one space dimension by Liu and Zeng [16].

Now, as we shall see in Lemma 3.1 and Theorem 3.2 for the linearized Navier-Stokes system, the lowest order term arising in the expansion of $\widehat{G}$ near $|\xi|=\infty$ is again a delta function, just as in the one-dimensional case. On the other hand, the complex analytic argument used in [22] to bound the remainder $G_{1}$ cannot be applied here, owing to a failure of analyticity. More specifically, in the one-dimensional case, Zeng writes a spectral decomposition for $\hat{G}_{1}$ in the 
Fourier inversion integral for $G$, moves the contour of integration into the upperhalf complex plane in an appropriate way, then derives the necessary pointwise bounds. This device depends upon the fact that the projection operators in the spectral decomposition for $\widehat{G}$ are analytic in the complex variable $\xi$, except at two resonant points on the real axis, which can be avoided in the integration. This argument appears to fail in several space variables, however, because the projection operators associated with the complexified transform $\widehat{G}$ now have an entire surface $(\xi+i \eta) \cdot(\xi+i \eta)=$ constant of singularities extending throughout the imaginary half-space; these cannot be avoided by moving the contour of integration. Moreover, there appears to be no particular advantage to such a decomposition in higher dimensions, because the support of $G$ is no longer concentrated near the characteristic cone. For example, setting $\varepsilon$ and $\eta$ to zero, we find that $G$ becomes the Green's matrix for the linearized Euler equations, which in the density component is exactly that of the scalar wave equation; in even dimensions, the sharp Huygen's principle fails for the wave equation. The best we can salvage from the complex-analytic approach is the analog of a weak Huygen's principle, that the support of $G$ is concentrated in the interior of the characteristic cone. Even this requires some extra work, due to loss of integrability in error terms of order $|\xi|^{-2}$.

Accordingly, we follow a different route in estimating $G$. Namely, we separate out from $\widehat{G}$ not just its behavior at $\xi=\infty$ (corresponding to a delta function), but its behavior in a neighborhood of infinity by setting $G=G_{1}+G_{2}$, where $\hat{G}_{1}(\xi, t)=\widehat{G}(\xi, t) \chi(\xi)$, and $\chi(\xi)$ is a smooth cutoff function supported on $|\xi| \leq R . G_{1}$ can then be estimated by comparison with the Green's matrix $\tilde{G}$ of the associated artificial viscosity system; the difficulty is therefore shifted to the term $G_{2}$. To be more specific, the transform $\hat{G}$ of the Green's matrix in question (and indeed that for any second order, isotropic system) has the form

$$
\widehat{G}(\xi, t)=h_{1}(|\xi|, t) I+h_{2}(|\xi|, t) M\left(\frac{\xi}{|\xi|}\right)
$$

where the entries of the matrix $M$ are quadratic polynomials in $\xi /|\xi|$. Now, the Fourier transform of the function $\xi /|\xi|$ is the distribution given by the Riesz kernel

$$
\mathcal{R} \stackrel{\text { def }}{=} \text { P.V. } C_{n} \frac{x}{|x|^{n+1}},
$$

so that the Green's matrix $G$ includes the convolution operator $u \mapsto \mathcal{R} * u$, which is not bounded from $L^{1}$ to $L^{1}$, nor from $L^{\infty}$ to $L^{\infty}$. Though it happens that $h_{2}(\infty, t) \equiv 0$, this operator must be reckoned with in higher order terms, $\mathcal{O}\left(|\xi|^{-1}\right)$. A careful analysis of the interplay between this operator and other, diffusive, effects in $G_{2}$ is needed to deal with these terms. The requisite Fourier 
multiplier theory developed in Section 4 and its application in Section 5, we regard as one of the most interesting aspects of this paper. Using these tools, we develop a result roughly analogous to that of Zeng: that

$$
G_{2}=e^{-b t} \delta\left(|x|-q^{*} t\right)\left[\begin{array}{ll}
1 & 0 \\
0 & 0
\end{array}\right]+e^{-b t} m(x, t),
$$

where $m \in L^{1}\left(\mathbb{R}^{n}\right)$ satisfies

$$
|m(x)| \leq \begin{cases}C|x|^{-n+1} & |x| \leq R \\ C(N)|x|^{-N} & |x|>R\end{cases}
$$

for any integer $N$. Thus, $G_{2}$ is concentrated primarily along particle paths. The fact that the decay of $G_{2}$ is algebraic as $|x| \rightarrow 0$ appears to be not a technical artifact but rather a natural consequence of the decomposition $W=W_{f}+W_{\perp}$ mentioned earlier in connection with the decay to an incompressible flow.

Plan of the paper. In Section 2 we give a complete theory of nonlinear, "artificial viscosity" systems. These are equations of the form

$$
u_{t}+f_{j}(u)_{x_{j}}=B_{j k} u_{x_{j} x_{k}},
$$

which are strictly parabolic and for which the symbols $e^{-\xi_{j} \xi_{k} B_{j k} t}$ and $e^{-i \xi_{j} f_{j}^{\prime}(0) t}$ commute. We are primarily interested in the nonlinear artifical viscosity system (1.5); general systems may be treated with little increase in complexity, however, and this we do. The results of Section 2 extend in a significant way the work of Hoff and Smoller [5].

In Section 3, we begin our study of the Navier-Stokes system (1.1) by deriving a complete frequency-domain description of the Green's matrix $G$ for the corresponding linearized equations. By uncoupling these equations, we can factor out the rotational invariance of the problem to obtain an explicit description of $\widehat{G}$ and bounds on all $\xi$-derivatives, $D_{\xi}^{\alpha} \widehat{G}(\xi, t)$, at both $|\xi|=0$ and $|\xi|=\infty$. In Section 4, we develop certain facts about Fourier multipliers, and in Section 5 we combine these facts with the bounds obtained in Section 3 to derive a decomposition of the Green's matrix into the sum

$$
G=G_{1}+G_{2}
$$

where $\left|\widehat{G}_{1}\right| \leq C e^{-\vartheta|\xi|^{2} t}$ is a strictly parabolic term, and $G_{2}=e^{-b t} \widehat{M}(\xi, t)$ is an exponentially decaying $L^{p}$ multiplier. Then in Theorem 5.3 we apply this decomposition to establish the $L^{p}$ decay (1.6)-(1.7) of solutions of (1.1) for $p \in[2, \infty]$. In Section 6, we give a definition of effective artificial viscosity, and we show 
that (1.3) is the unique effective artificial viscosity system whose solutions are asymptotically equivalent to those of (1.1). Combining this with the estimates of Section 5, we then obtain our results concerning the incompressible limit and the decay to diffusion waves discussed earlier.

In Section 7, we begin the study of $L^{p}$ behavior in the low norms $1 \leq$ $p<2$, by deriving pointwise bounds on $G(x, t)$. First we give some results for fairly general systems which are strictly parabolic and whose convective parts are symmetrizable. We establish for such systems the pointwise estimate

$$
|G(x, t)| \leq C e^{-\left((|x|-\Lambda t)^{+}\right)^{2} / 4 \mu t},
$$

where $\Lambda$ is a bound for the characteristic speeds of the associated hyperbolic system. (1.16) is thus a weaker version of the Paley-Wiener theorem. When the system additionally exhibits the commmutativity of an artifical viscosity system, we can factor out the hyperbolic effects and derive even stronger estimates. On the other hand, if the system is not strictly parabolic, such as is the case for the linearized Navier-Stokes equations, this entire complex-analytic approach fails. We can still obtain the somewhat weaker bounds

$$
|G(x, t)| \leq C_{n}\left(\frac{|x|}{1+t}\right)^{-n}, \quad|x|>0,
$$

however, directly from the derivative estimates for $D_{\xi} \widehat{G}$ derived in Section 3, together with standard properties of the Fourier transform. Finally in Section 8, we apply the results of Section 7 to derive rates of decay in $L^{p}$ for $p \in[1,2)$. To do this we first describe the behavior of the smooth part $G_{1}$ of the Green's function $G$ by comparing with the Green's function $\tilde{G}=G^{B} * W$ of the associated effective artificial viscosity system (1.3). The residual $\left(G_{1}-\tilde{G}\right)$ satisfies only the weak pointwise bound (1.17), but decays much faster than $\tilde{G}$ in $L^{2}$. Combining these facts, we find that $\left\|G_{1}-\tilde{G}\right\|_{p}$ is still dominated by $\|\tilde{G}\|_{p}$, for $1 \leq p<2$. This information, together with the previous description of $G_{2}$ derived in Sections 3 and 4 , is sufficient to give decay and asymptotic behavior of the solution in $L^{p}$ for $p \in[1,2)$.

2. Nonlinear systems with artificial viscosity. The nonlinear artificial viscosity system (1.5) is of particular importance for the Navier-Stokes equations (1.1). We therefore collect together here various properties of its solutions. Actually, much more general systems may be treated, with little increase in complexity. We therefore study in this section nonlinear systems with constant viscosity terms:

$$
u_{t}+f_{j}(u)_{x_{j}}=B_{j k} u_{x_{j} x_{k}} .
$$


Here $u: \mathbb{R}^{n} \times[0, \infty) \rightarrow \mathbb{R}^{m}$ is an unknown vector function of $x \in \mathbb{R}^{n}$ and $t \in[0, \infty) ; B_{j k}$ is a constant $m \times m$ matrix; and $f_{j}$ maps a ball in $\mathbb{R}^{m}$ into $\mathbb{R}^{m}$. In Theorem 2.2 below we prove the global existence of solutions for initial data which is bounded in $L^{\infty}$ and small in $L^{2}$, requiring only that (2.1) be symmetrizable and strictly parabolic. Then in Theorem 2.3 , under the further assumption that the symbol associated with the hyperbolic part of the equation commutes with that of its parabolic part, we show that the solution $u$ decays at the optimal rate in $W^{k, p}$ for $p \in[2, \infty]$, and, moreover, that $u(\cdot, t)$ is wellapproximated by the solution of the corresponding linearized equation, for large $t$.

We assume that $f_{j}$ is defined and $C^{1}$ in a closed ball $\overline{B_{r_{0}}(0)} \subseteq \mathbb{R}^{m}$, and we denote by $A_{j}$ the Jacobian matrix $f_{j}^{\prime}(0)$. The linearization of $(2.1)$ is therefore the system

$$
u_{t}+A_{j} u_{x_{j}}=B_{j k} u_{x_{j} x_{k}} .
$$

In the following theorem we derive a number of important properties of the Green's matrix corresponding to the linearized system (2.2).

Theorem 2.1. (a). Assume that there is a symmetric, positive definite matrix $P$ such that $P A_{j}$ is symmetric for each $j$, and let $\kappa=\left|P^{1 / 2}\right|\left|P^{-1 / 2}\right|$. Then for $t \in \mathbb{R}, \xi \in \mathbb{R}^{n}$, and $w \in \mathbb{R}^{m}$,

$$
\kappa^{-1}|w| \leq\left|e^{-i \xi_{j} A_{j} t} w\right| \leq \kappa|w| .
$$

(b) Assume, in addition, that there is a positive number $\varepsilon$ such that

$$
\left\langle\xi_{j} \xi_{k} P B_{j k} w, w\right\rangle_{\mathbb{R}^{m}} \geq \varepsilon|\xi|^{2}|w|^{2}
$$

for all $\xi \in \mathbb{R}^{n}$ and $w \in \mathbb{R}^{m}$. Then for $t>0$ the components of the matrix functions $\widehat{G}(\xi, t)=e^{-\left(i \xi_{j} A_{j}+\xi_{j} \xi_{k} B_{j k}\right) t}$ and $\widehat{G}^{B}(\xi, t)=e^{-\xi_{j} \xi_{k} B_{j k} t}$ are in the Schwartz class $\mathcal{S}\left(\mathbb{R}^{m}\right)$, and therefore so are those of the transforms $G(\cdot, t)$ and $G^{B}(\cdot, t)$. Moreover, for each nonnegative integer $k$ there is a positive constant $C(k)$ such that

$$
\left\|D_{x}^{\alpha} G^{B}(\cdot, t)\right\|_{p} \leq C(k) t^{n / 2(1 / p-1)-|\alpha| / 2},|\alpha| \leq k, p \in[1, \infty], t>0 ;
$$

and

$$
\begin{aligned}
& \left\|D_{x}^{\alpha} G(\cdot, t)\right\|_{p} \leq C(k) t^{n / 2(1 / p-1)-|\alpha| / 2}, \\
& \qquad|\alpha| \leq k, \begin{cases}p \in[2, \infty] & t>0, \quad \text { or } \\
p \in[1,2], & 0<t \leq 1 .\end{cases}
\end{aligned}
$$


Finally, there is a constant $C_{1}$ such that

$$
\left\|G^{B}(\cdot, t)\right\|_{1}=C_{1}, t>0 .
$$

Proof: To prove (a) we observe that the matrix $S_{j}=P^{-1 / 2}\left(P A_{j}\right) P^{-1 / 2}$ is symmetric and that $P^{-1 / 2} S_{j} P^{1 / 2}=A_{j}$. The matrix $e^{-i \xi_{j} S_{j} t}$ is therefore unitary, and $e^{-i \xi_{j} A_{j} t}=P^{-1 / 2} e^{-i \xi_{j} S_{j} t} P^{1 / 2}$. This proves (2.3).

Next we show that there are positive constants $C$ and $\vartheta$ such that

$$
|\widehat{G}(\xi, t)|,\left|\widehat{G}^{B}(\xi, t)\right| \leq C e^{-\vartheta|\xi|^{2} t} .
$$

For the proof of (2.8) we temporarily let $\widehat{G}$ denote either $\widehat{G}$ or $\widehat{G}^{B}$. Fix $\xi \in \mathbb{R}^{n}$ and $w_{0} \in \mathbb{C}^{m}$ and set $w(t)=\widehat{G}(\xi, t) w_{0}$. Then

$$
\dot{w}=-\left(i \xi_{j} A_{j}+\xi_{j} \xi_{k} B_{j k}\right) w .
$$

A routine computation based on the symmetry of $S_{j}$ and on (2.4) shows that $v=P^{1 / 2} w$ satisfies

$$
\frac{d}{d t}|v|^{2}=\langle\dot{v}, v\rangle_{\mathbb{C}^{m}}+\langle v, \dot{v}\rangle_{\mathbb{C}^{m}} \leq-2 \varepsilon|\xi|^{2}|w|^{2} .
$$

It follows that

$$
\left|\widehat{G}(\xi, t) w_{0}\right|=|w(t)| \leq C e^{-\vartheta|\xi|^{2} t}\left|w_{0}\right|,
$$

which proves (2.8). The statements in (2.5) and (2.6) for $p \geq 2$ follow immediately, for if $q$ is the Hölder conjugate of $p$, then

$$
\left\|D_{x}^{\alpha} G(\cdot, t)\right\|_{p} \leq C\left\|\xi^{\alpha} \widehat{G}(\xi, t)\right\|_{q} \leq C\left[\int|\xi|^{|\alpha| q} e^{-q \vartheta|\xi|^{2} t} d \xi\right]^{1 / q}
$$

The $p<2$ bounds in (2.5), as well as $(2.7)$, follow from the fact that $G^{B}(\cdot, 1) \in$ $\mathcal{S} \subseteq L^{1}$ and the scaling property

$$
G^{B}(x, t)=t^{-n / 2} G^{B}\left(t^{-1 / 2} x, 1\right),
$$

which is evident from the Fourier integral representation of $G^{B}$ in terms of its transform $\widehat{G}^{B}=e^{-\xi_{j} \xi_{k} B_{j k} t}$.

To prove the $p<2$ estimates in (2.6) for $G$, we first show that there is a constant $C$ such that

$$
\|G(\cdot, t)\|_{1} \leq C, \quad 0<t \leq 1 .
$$


To see this, we choose $u_{0} \in \mathcal{S}$ and define $u(\cdot, t)=G(\cdot, t) * u_{0} \in \mathcal{S}$. $u$ is a solution of (2.2) and so satisfies the alternative representation

$$
u(\cdot, t)=G^{B}(\cdot, t) * u_{0}-\int_{0}^{t} G_{x_{j}}^{B}(\cdot, t-s) *\left(A_{j} u(\cdot, s)\right) d s .
$$

Now, an easy argument based on (2.8) and the fact that $u_{0} \in \mathcal{S}$ shows that $\mathcal{B}(t)=\sup _{0 \leq s \leq t}\|u(\cdot, s)\|_{1}$ is finite. On the other hand, from (2.5) and (2.10), we find that, for $s \in[0, t]$,

$$
\|u(\cdot, s)\|_{1} \leq C\left[\left\|u_{0}\right\|_{1}+t^{1 / 2} \mathcal{B}(t)\right] .
$$

A simple Gronwall-type argument then shows that

$$
\left\|G(\cdot, t) * u_{0}\right\|_{1}=\|u(\cdot, t)\|_{1} \leq C\left\|u_{0}\right\|_{1}, \quad 0<t \leq 1 .
$$

We now replace $u_{0}$ by a sequence of mollifying kernels converging to the $\delta$ distribution, multiplied by standard basis vectors, to obtain (2.9). A similar argument, based on (2.5) and (2.9), then shows that

$$
\left\|G_{x_{k}}(\cdot, t)\right\|_{1} \leq C t^{-1 / 2}, \quad 0<t \leq 1 .
$$

To obtain estimates for higher-order derivatives, we simply write

$$
D_{x}^{\alpha} G(\cdot, t)=\frac{\partial}{\partial x_{j_{1}}} G\left(\cdot,|\alpha|^{-1} t\right) * \frac{\partial}{\partial x_{j_{2}}} G\left(\cdot,|\alpha|^{-1} t\right) * \cdots * \frac{\partial}{\partial x_{j_{|\alpha|}}} G\left(\cdot,|\alpha|^{-1} t\right)
$$

and apply (2.11) to each factor. This proves (2.6) for $p=1$; the bounds for $1<p<2$ then follow by interpolation.

We now make a few remarks concerning the symmetrizability hypothesis in Theorem 2.1a. We say that $S \in C^{2}\left(\overline{B_{r_{0}}(0)}\right)$ is a convex entropy for (2.1) if $S^{\prime \prime}(u)>0$ for $u \in \overline{B_{r_{0}}(0)}$ and if each of the vector functions $\left(f_{j}^{\prime}\right)^{t} \nabla_{u} S$ is the gradient with respect to $u$ of a scalar function in $\overline{B_{r_{0}}(0)}$. The fundamental observation of Lax and Friedrichs [10] and Godunov [1] is that the existence of a convex entropy implies the symmetrizability of the nonlinear system (2.1): if $S$ is the entropy, then each of the matrices $S^{\prime \prime}(u) f_{j}^{\prime}(u)$ is symmetric. In particular, setting $u=0$, we find that the symmetric, positive definite matrix $P=S^{\prime \prime}(0)$ symmetrizes the $A_{j}$ in the sense of Theorem 2.1a. As is well known, this symmetrizability implies a global $L^{2}$ bound for solutions of (2.1); this fact will be crucial in extending local solutions for all time. 
Given initial data $u_{0} \in L^{\infty}\left(\mathbb{R}^{n}\right)$ with $\left\|u_{0}\right\|_{\infty} \leq r_{0}$, we say that $u$ is a solution of $(2.1)$ on $[0, T]$ with data $u_{0}$ provided

$$
\left\{\begin{array}{l}
u \in C\left([0, T] ; L_{\mathrm{loc}}^{1}\left(\mathbb{R}^{n}\right)\right), u(\cdot, 0)=u_{0} ; \\
u(\cdot, t) \in \overline{B_{r_{0}}(0)} \quad \text { a.e.; } \\
u(\cdot, t)=G^{B}(\cdot, t) * u_{0}-\int_{0}^{t} G_{x_{j}}^{B}(\cdot, t-s) * f_{j}(u(\cdot, s)) d s,
\end{array}\right.
$$

where $G^{B}$ is the Green's matrix defined in Theorem 2.1b. In the following theorem we prove the global existence of such solutions when there is a convex entropy and when the initial data is small in $L^{2}$.

Theorem 2.2. Let $k$ be a positive integer, and assume that:

$$
f_{j} \in C^{k}\left(\overline{B_{r_{0}}(0)}\right)
$$

there is a convex entropy $S$ for the system (2.1) satisfying $S^{\prime \prime}>0$ on $\overline{B_{r_{0}}(0)}$,

$$
\text { the parabolicity (2.4) holds with } P=S^{\prime \prime}(0) \text {. }
$$

Let $C_{1}$ be as in (2.7). Then given $r<r_{0}$ there are positive constants $C$ and $\mu$ such that, if

$$
\left\|u_{0}\right\|_{\infty} \leq \frac{r}{C_{1}} \text { and }\left\|u_{0}\right\|_{2} \leq \mu
$$

then there is a unique global solution $u$ of the system (2.1). u satisfies

$$
u \in C\left((0, \infty) ; W^{k, p}\left(\mathbb{R}^{m}\right)\right), \quad p \in[2, \infty],
$$

and

$$
\left\|D_{x}^{\alpha} u(\cdot, t)\right\|_{p} \leq C \max \left\{1, t^{-|\alpha| / 2}\right\}\left\|u_{0}\right\|_{p}
$$

for $|\alpha| \leq k$ and $p \in[2, \infty]$. If $u_{0}$ is additionally in $L^{p_{0}}$ for $1 \leq p_{0} \leq 2$, then (2.16) and (2.17) hold for $p \in\left[p_{0}, \infty\right]$. 
Proof. The proof is fairly routine, given the properties of $G^{B}$ listed in Theorem 2.1. We therefore omit most of the technical details. First we define a sequence of approximate solutions $u^{\ell}$ by taking $u^{0}=0$ and $u^{\ell+1}=\mathcal{L}\left(u^{\ell}\right)$, where $\mathcal{L}(u)$ is the expression on the right-hand side of the third condition in (2.12). Standard estimates based on (2.5) and (2.7) then show that, if $\tau>0$ is sufficiently small, then each $u^{\ell}(\cdot, t)$ has values in $\overline{B_{r_{0}}(0)}$ for $0 \leq t \leq \tau$, and that the $u^{\ell}$ converge uniformly on $\mathbb{R}^{n} \times(0, \tau]$ to a unique solution $u \in C\left(\mathbb{R}^{n} \times(0, \tau]\right)$. An easy induction argument proves that

$$
\left\|D_{x}^{\alpha} u^{\ell}(\cdot, t)\right\|_{p} \leq C_{2} t^{-|\alpha| / 2}\left\|u_{0}\right\|_{p}
$$

for $t \leq \tau,|\alpha| \leq k$, and $p \in\left[p_{0}, \infty\right]$, and straightforward weak convergence considerations show that the solution $u$ inherits these properties. We fix the time $\tau$ and the constant $C_{2}$ in (2.18), and we extend the solution to all of $t>0$ as follows. First, as is well known, the existence of the entropy $S$ implies a time-independent $L^{2}$ energy estimate: there is a constant $C_{3}$ such that, if $u$ is a solution on $[0, T]$ with values in $\overline{B_{r_{0}}(0)}$, then

$$
\|u(\cdot, t)\|_{2} \leq C_{3}\left\|u_{0}\right\|_{2}, \quad 0 \leq t \leq T .
$$

Then by (2.18) and (2.19), for $p>n$,

$$
\begin{aligned}
\|u(\cdot, T)\|_{\infty} & \leq C\|u(\cdot, T)\|_{W^{1, p}} \\
& \leq C\left[\|u(\cdot, T)\|_{p}+C_{2}\left(\frac{1}{2} \tau\right)^{-1 / 2}\left\|u\left(\cdot, T-\frac{1}{2} \tau\right)\right\|_{p}\right] \\
& \leq C r_{0}^{(p-2) / p}\left[\|u(\cdot, T)\|_{2}^{2 / p}+C_{2}\left(\frac{1}{2} \tau\right)^{-1 / 2}\left\|u\left(\cdot, T-\frac{1}{2} \tau\right)\right\|_{2}^{2 / p}\right] \\
& \leq C C_{3}^{2 / p} r_{0}^{(p-2) / p}\left[1+C_{2}\left(\frac{1}{2} \tau\right)^{-1 / 2}\right]\left\|u_{0}\right\|_{2}^{2 / p} \\
& \leq r
\end{aligned}
$$

if $\left\|u_{0}\right\|_{2}$ is sufficiently small. We may then reapply our local existence result at the new initial time $T$, and in this way extend the solution to all of $t>0$. The estimate (2.17) follows from (2.18), and the continuity assertion (2.16) follows from the representation $u=\mathcal{L}(u)$.

To obtain more detailed information about the large time behavior of $u$, we shall have to take into account more of the structure of the flux functions $f_{j}$. We therefore linearize each $f_{j}$ about $u=0$ :

$$
f_{j}(u)=A_{j} u-Q_{j}(u)
$$


where, as before, $A_{j}=f_{j}^{\prime}(0)$, and

$$
\left|Q_{j}(u)\right| \leq C|u|^{2}, \quad|u| \leq r_{0} .
$$

Note that $Q_{j}$ is $C^{k}$ if $f$ is $C^{k+2}$. We substitute (2.20) into the representation (2.12), take Fourier transforms, then solve the resulting ode in terms of the fundamental matrix $e^{-\left(i \xi_{j} A_{j}+\xi_{j} \xi_{k} B_{j k}\right) t}$. Transforming back, we obtain the following alternative representation for the solution $u$ of Theorem 2.2:

$$
u(\cdot, t)=G(\cdot, t) * u_{0}+\int_{0}^{t} G_{x_{j}}(\cdot, t-s) * Q_{j}(u(\cdot, s)) d s .
$$

In the following theorem, we shall assume that, for each $\xi \in \mathbb{R}^{n}$, the matrices $\xi_{j} A_{j}$ and $\xi_{j} \xi_{k} B_{j k}$ commute, so that

$$
\widehat{G}(\xi, t)=e^{-\left(i \xi_{j} A_{j}+\xi_{j} \xi_{k} B_{j k}\right) t}=e^{-i \xi_{j} A_{j} t} \widehat{G}^{B}(\xi, t) .
$$

Now, (2.3) shows that, for each fixed $t$, the function $\xi \mapsto e^{-i \xi_{j} A_{j} t}$ is in $L^{\infty}\left(\mathbb{R}^{n}\right)$, and therefore is the Fourier transform of a tempered distribution, which we denote by $(2 \pi)^{n / 2} W(t)$. Evidently,

$$
\kappa^{-1}\|\varphi\|_{2} \leq\|W(t) * \varphi\|_{2} \leq \kappa\|\varphi\|_{2}
$$

for all $\varphi \in L^{2}$, where $\kappa$ is as in (2.3). In addition, the convolution theorem applied in (2.22) gives the very useful factorization

$$
G(\cdot, t)=W(t) * G^{B}(\cdot, t) .
$$

We now give the main theorem of this section, which establishes optimal rates of decay in $W^{k, p}$ for small-data solutions of (2.1):

Theorem 2.3. Let $k$ be a positive integer, assume that (2.14) and (2.15) hold, that

$$
f_{j} \in C^{k+2}\left(\overline{B_{r_{0}}(0)}\right)
$$

and that

$$
\xi_{j} A_{j} \text { and } \xi_{j} \xi_{k} B_{j k} \text { commute, for all } \xi \in \mathbb{R}^{n} .
$$

Let $C_{1}$ be as in (2.7). Then, given $r<r_{0}$, there are positive constants $C$ and $\mu$ such that, if

$$
\left\|u_{0}\right\|_{\infty} \leq \frac{r}{C_{1}} \text { and }\left\|u_{0}\right\|_{1} \leq \mu,
$$

then the corresponding solution $u$ of (2.1) satisfies

$$
\left\|D_{x}^{\alpha} u(\cdot, t)\right\|_{p} \leq C t^{n / 2(1 / p-1)-|\alpha| / 2}\left\|u_{0}\right\|_{1}
$$

and

$$
\begin{array}{r}
\left\|D_{x}^{\alpha}\left(u-G(\cdot, t) * u_{0}\right)\right\|_{p} \leq C t^{n / 2(1 / p-1)-|\alpha| / 2-1 / 2} \\
\times\left[\left\|u_{0}\right\|_{2}^{2}+L(t)\left\|u_{0}\right\|_{1}^{2}\right],
\end{array}
$$


where $L$ is the function defined by $L(t)=\log t$ when $n=2$, and $L(t)=1$ when $n \geq 3$. (2.27) and (2.28) hold for $t \geq 1, p \in[2, \infty]$, and $|\alpha| \leq k$.

Proof. First we let $u_{I}=G * u_{0}$ and $u_{I I}=u-u_{I}$. For $T \geq 1$ we define

$$
A(T)=\sup _{1 \leq t \leq T} \sup _{\substack{0 \leq|\alpha| \leq k \\ 2 \leq p \leq \infty}} t^{r|\alpha|, p}\left\|D_{x}^{\alpha} u(\cdot, t)\right\|_{p}
$$

where

$$
r_{\ell, p}=\frac{n}{2}\left(1-\frac{1}{p}\right)+\frac{\ell}{2}
$$

We shall estimate $A(T)$ in terms of $\left\|u_{0}\right\|_{1}$ and $A(T)^{2}$, treating $u_{I}$ and $u_{I I}$ separately. The required bounds for $u_{I}$ follow directly from (2.6):

$$
\left\|D_{x}^{\alpha} u_{I}(\cdot, t)\right\|_{p} \leq\left\|D_{x}^{\alpha} G(\cdot, t)\right\|_{p}\left\|u_{0}\right\|_{1} \leq C t^{-r_{|\alpha|, p}}\left\|u_{0}\right\|_{1} .
$$

The estimates for the term

$$
D_{x}^{\alpha} u_{I I}(\cdot, t)=\int_{0}^{t} D_{x}^{\alpha}\left[G_{x_{j}}^{B}(\cdot, t-s) * W(t-s) * Q_{j}(u(\cdot, s))\right] d s
$$

are somewhat more involved. First, if $t \in[1,2]$, then straightforward arguments based on the bounds (2.17) of Theorem 2.2 show that

$$
\left\|D_{x}^{\alpha} u_{I I}(\cdot, t)\right\|_{p} \leq C\left\|u_{0}\right\|_{2}^{2} \leq C\left\|u_{0}\right\|_{2}^{2} t^{-r_{|\alpha|, p}-1 / 2} .
$$

Now assume that $t \in[2, T]$. We let $\mathcal{I}(x, t)$ denote the integrand in (2.30), and we estimate $\int_{0}^{t / 2} \mathcal{I}$ and $\int_{t / 2}^{t} \mathcal{I}$ separately. Applying (2.23), (2.26), and (2.5), we may bound the first of these by

$$
\begin{aligned}
& \int_{0}^{t / 2}\left\|G_{x_{j}}^{B}\left(\cdot, \frac{1}{2}(t-s)\right) * W(t-s) * D_{x}^{\alpha} G^{B}\left(\cdot, \frac{1}{2}(t-s)\right) * Q_{j}(u(\cdot, s))\right\|_{p} d s \\
\leq & \int_{0}^{t / 2}\left\|G_{x_{j}}^{B}\left(\cdot, \frac{1}{2}(t-s)\right)\right\|_{2 p /(p+2)}\left\|D_{x}^{\alpha} G^{B}\left(\cdot, \frac{1}{2}(t-s)\right)\right\|_{2}\left\|Q_{j}(u(\cdot, s))\right\|_{1} d s \\
\leq & \int_{0}^{t / 2}(t-s)^{-r_{|\alpha|, p}-1 / 2}\left\|Q_{j}(u(\cdot, s))\right\|_{1} d s .
\end{aligned}
$$


For $s \in[0,1],\left\|Q_{j}\right\|_{1} \leq C\left\|u_{0}\right\|_{2}^{2}$, by $(2.17) ;$ and for $s \in[1, t / 2],\left\|Q_{j}\right\|_{1} \leq$ $C\|u(\cdot, s)\|_{2}^{2} \leq C s^{-n / 2} A(T)^{2}$. Thus

$$
\begin{aligned}
& \left\|\int_{0}^{t / 2} \mathcal{I}(\cdot, s) d s\right\|_{p} \\
& \leq C\left[\left\|u_{0}\right\|_{2}^{2} \int_{0}^{1}(t-s)^{-r_{|\alpha|, p}-1 / 2}+A(T)^{2} \int_{1}^{t / 2}(t-s)^{-r_{|\alpha|, p}-1 / 2} s^{-n / 2} d s\right] \\
& \leq C t^{-r_{|\alpha|, p}-1 / 2}\left[\left\|u_{0}\right\|_{2}^{2}+L(T) A(T)^{2}\right],
\end{aligned}
$$

where $L$ is as in the statement of the theorem.

Before deriving estimates for $\int_{t / 2}^{t} \mathcal{I}$, we first observe that, for $|\alpha| \leq k$, each component of the vector $D_{x}^{\alpha} Q_{j}(u)$ is a sum of terms of the form $a(u)\left(D_{x}^{\gamma_{1}} u^{j_{1}}\right) \ldots$ $\left(D_{x}^{\gamma_{r}} u^{j_{r}}\right)$, where $a \in L^{\infty}\left(\overline{B_{r_{0}}(0)}\right), 2 \leq r \leq k$, and $\left|\gamma_{1}\right|+\cdots+\left|\gamma_{r}\right|=|\alpha|$. Thus

$$
\begin{aligned}
\left\|D_{x}^{\alpha} Q_{j}(u(\cdot, t))\right\|_{p} & \leq C\left\|D_{x}^{\gamma_{1}} u\right\|_{\infty} \cdots\left\|D_{x}^{\gamma_{r-1}} u\right\|_{\infty}\left\|D_{x}^{\gamma_{r}} u\right\|_{p} \\
& \leq C t^{n / 2(1 / p-2)-|\alpha| / 2}\left[A(T)^{2}+A(T)^{k}\right] .
\end{aligned}
$$

We shall give estimates for $\int_{t / 2}^{t} \mathcal{I}$ in $L^{2}$ and $L^{\infty}$, and then interpolate. First, we obtain from (2.32), (2.23), and (2.5) that

$$
\begin{aligned}
\left\|\int_{t / 2}^{t} \mathcal{I}(\cdot, s) d s\right\|_{2} & \leq \int_{t / 2}^{t}\left\|G_{x_{j}}^{B}(\cdot, t-s)\right\|_{1}\left\|D_{x}^{\alpha} Q_{j}(u(\cdot, s))\right\|_{2} d s \\
& \leq C\left[\int_{t / 2}^{t}(t-s)^{-1 / 2} s^{-3 n / 4-|\alpha| / 2} d s\right]\left[A(T)^{2}+A(T)^{k}\right] \\
& \leq C t^{-r_{|\alpha|, 2}-1 / 2}\left[A(T)^{2}+A(T)^{k}\right]
\end{aligned}
$$

since $n \geq 2$. To estimate $\left\|\int_{t / 2}^{t} \mathcal{I}\right\|_{\infty}$ we split further: first, by (2.23), (2.32), and (2.5),

$$
\begin{array}{rl}
\| \int_{t / 2}^{t-1} & \mathcal{I}(\cdot, s) d s\left\|_{\infty} \leq \int_{t / 2}^{t-1}\right\| G_{x_{j}}^{B}(\cdot, t-s)\left\|_{2}\right\| D_{x}^{\alpha} Q_{j}(u(\cdot, s)) \|_{2} d s \\
& \leq C\left[\int_{t / 2}^{t-1}(t-s)^{-n / 4-1 / 2} s^{-3 n / 4-|\alpha| / 2} d s\right]\left[A(T)^{2}+A(T)^{k}\right] \\
& \leq C t^{-r_{|\alpha|, \infty}-1 / 2}\left[A(T)^{2}+A(T)^{k}\right]
\end{array}
$$


and by (2.6) and (2.32),

$$
\begin{aligned}
\left\|\int_{t-1}^{t} \mathcal{I}(\cdot, s) d s\right\|_{\infty} & \leq \int_{t-1}^{t}\left\|G_{x_{j}}(\cdot, s) * D_{x}^{\alpha} Q_{j}(u(\cdot, s))\right\|_{\infty} d s \\
& \leq \int_{t-1}^{t}\left\|G_{x_{j}}(\cdot, t-s)\right\|_{1}\left\|D_{x}^{\alpha} Q_{j}(u(\cdot, s))\right\|_{\infty} d s \\
& \leq C\left[\int_{t-1}^{t}(t-s)^{-1 / 2} s^{-n-|\alpha| / 2} d s\right]\left[A(T)^{2}+A(T)^{k}\right] \\
& \leq C t^{-r_{|\alpha|, \infty}-1 / 2}\left[A(T)^{2}+A(T)^{k}\right] .
\end{aligned}
$$

Adding (2.34) and (2.35), we then obtain

$$
\left\|\int_{t / 2}^{t} \mathcal{I}(\cdot, s) d s\right\|_{\infty} \leq C t^{-r_{|\alpha|, \infty}-1 / 2}\left[A(T)^{2}+A(T)^{k}\right] .
$$

Interpolating (2.33) and (2.36), we then conclude that

$$
\left\|\int_{t / 2}^{t} \mathcal{I}(\cdot, s) d s\right\|_{p} \leq C t^{-r_{|\alpha|, p}-1 / 2}\left[A(T)^{2}+A(T)^{k}\right] .
$$

This together with (2.31) shows that

$$
\left\|D_{x}^{\alpha} u_{I I}(\cdot, t)\right\|_{p} \leq C t^{-r_{|\alpha|, p}-1 / 2}\left[\left\|u_{0}\right\|_{2}^{2}+L(T) A(T)^{2}+A(T)^{k}\right] .
$$

Combining (2.37) with the estimate (2.29) for $u_{I}$ and taking the appropriate sup over $t$, we then obtain that

$$
A(T) \leq C\left[\left\|u_{0}\right\|_{1}+A(T)^{2}+A(T)^{k}\right] .
$$

A routine argument based on the continuity (2.16) of $A$ in $T$ then shows that $A(T) \leq C\left\|u_{0}\right\|_{1}$, for all $T$, provided that $\left\|u_{0}\right\|_{1}$ is sufficiently small. This proves (2.27), and (2.28) follows by substituting this bound for $A$ into (2.37).

We conclude this section with an example showing that the decay rate (2.27) is sharp for $p=2$. We simply take $u_{0}=G^{B}(\cdot, 1) \bar{u}$, where $\bar{u} \neq 0$ is a constant vector, which can be chosen so that $\left\|u_{0}\right\|_{1}$ and $\left\|u_{0}\right\|_{2}$ are arbitrarily small. Then, by (2.23) and (2.24), for $t \geq 1$,

$$
\begin{aligned}
\left\|D_{x}^{\alpha}\left[G(\cdot, t) * u_{0}\right]\right\|_{2} & =\left\|W(t) * D_{x}^{\alpha} G^{B}(\cdot, t+1) \bar{u}\right\|_{2} \\
& \geq C^{-1}\left[\int\left|\xi^{\alpha} e^{-\xi_{j} \xi_{k} B_{j k}(t+1)} \bar{u}\right|^{2} d \xi\right]^{1 / 2} \\
& =C^{-1} t^{-n / 4-|\alpha| / 2} .
\end{aligned}
$$


On the other hand, if $u(\cdot, t)$ is the solution of the nonlinear system (2.1) with this same initial data $u_{0}$, then by $(2.28)$,

$$
\left\|D_{x}^{\alpha}\left[u(\cdot, t)-G(\cdot, t) * u_{0}\right]\right\|_{2} \leq C t^{-n / 4-|\alpha| / 2-1 / 2} L(t) .
$$

We conclude that

$$
\left\|D_{x}^{\alpha} u(\cdot, t)\right\|_{2} \geq C^{-1} t^{-n / 4-|\alpha| / 2},
$$

which shows that (2.27) is sharp for $p=2$, as well as that

$$
\left\|D_{x}^{\alpha}\left[u(\cdot, t)-G(\cdot, t) * u_{0}\right]\right\|_{2} \leq C t^{-1 / 2} L(t)\|u(\cdot, t)\|_{2} .
$$

Thus in the limit as $t \rightarrow \infty$, small-data solutions of the nonlinear system (2.1) are well approximated by solutions of the corresponding linearized system (2.2).

\section{Fourier analysis of the Green's matrix for the linearized Navier-} Stokes system. In this section we begin the systematic study of the large-time behavior of solutions of the Navier-Stokes equations (1.1). In Lemma 3.1 below we give an explicit derivation of the Fourier transform $\widehat{G}(\xi, t)$ of the Green's matrix corresponding to the linearized system (1.4). Then in Theorem 3.2 we prove various pointwise bounds for $\widehat{G}$ and its $\xi$-derivatives, required for the decay analysis of Sections 4-8.

Lemma 3.1. The Fourier transform $\widehat{G}$ of the Green's matrix for the linearized Navier-Stokes system (1.4) is given by

$$
\widehat{G}(\xi, t)=\left[\begin{array}{cc}
\frac{\lambda_{+} e^{\lambda_{-} t}-\lambda_{-} e^{\lambda_{+} t}}{\lambda_{+}-\lambda_{-}} & -i\left(\frac{e^{\lambda_{+} t}-e^{\lambda_{-} t}}{\lambda_{+}-\lambda_{-}}\right) \xi^{t} \\
-i c^{2}\left(\frac{e^{\lambda_{+} t}-e^{\lambda_{-} t}}{\lambda_{+}-\lambda_{-}}\right) \xi & e^{-\varepsilon|\xi|^{2} t} I+\left(\frac{\lambda_{+} e^{\lambda_{+} t}-\lambda_{-} e^{\lambda_{-} t}}{\lambda_{+}-\lambda_{-}}-e^{-\varepsilon|\xi|^{2} t}\right) \frac{\xi \xi^{t}}{|\xi|^{2}}
\end{array}\right],
$$

where

$$
\lambda_{ \pm}=-\frac{1}{2} \nu|\xi|^{2} \pm \frac{1}{2} \sqrt{\nu^{2}|\xi|^{4}-4 c^{2}|\xi|^{2}}
$$

and $\nu=\varepsilon+\eta$. The representation above holds for $|\xi| \neq 0,2 c / \nu$.

Proof. We solve the linearized system (1.4) with initial data $\left(\rho_{0}, m_{0}\right)$ as follows. First, applying the operator $\sum_{j} \partial / \partial x_{j}$ to the second equation in (1.4), we obtain

$$
D_{t}+c^{2} \Delta \rho=\nu \Delta D
$$


where $D=\operatorname{div} m$. Combining this with the first equation in (1.4), we can isolate $\rho$ :

$$
\rho_{t t}=-D_{t}=c^{2} \Delta \rho+\nu \Delta \rho_{t} .
$$

Taking Fourier transforms, we then obtain the following initial-value problem for $\hat{\rho}(\xi, \cdot)$ :

$$
\left\{\begin{array}{l}
\hat{\rho}_{t t}+\nu|\xi|^{2} \hat{\rho}_{t}+c^{2}|\xi|^{2} \hat{\rho}=0 \\
\hat{\rho}(\xi, 0)=\hat{\rho}_{0}(\xi) \\
\hat{\rho}_{t}(\xi, 0)=-i \xi \cdot \widehat{m}_{0}(\xi) .
\end{array}\right.
$$

It is easily seen that $\lambda_{ \pm}(\xi)$ are the roots of the corresponding indicial equation, so that, when $\lambda_{-} \neq \lambda_{+}$,

$$
\hat{\rho}(\xi, t)=A(\xi) e^{\lambda_{-}(\xi) t}+B(\xi) e^{\lambda_{+}(\xi) t} .
$$

The initial conditions give that

$$
A=\frac{\lambda_{+} \hat{\rho}_{0}+i \xi \cdot \widehat{m}_{0}}{\lambda_{+}-\lambda_{-}} \quad \text { and } \quad B=\frac{-i \xi \cdot \widehat{m}_{0}-\lambda_{-} \hat{\rho}_{0}}{\lambda_{+}-\lambda_{-}}
$$

so that

$$
\hat{\rho}(\xi, t)=\left(\frac{\lambda_{+} e^{\lambda_{-} t}-\lambda_{-} e^{\lambda_{+} t}}{\lambda_{+}-\lambda_{-}}\right) \hat{\rho}_{0}(\xi)-i\left(\frac{e^{\lambda_{+} t}-e^{\lambda_{-} t}}{\lambda_{+}-\lambda_{-}}\right) \xi \cdot \widehat{m}_{0}(\xi) .
$$

This determines $\widehat{G^{11}}$ and $\widehat{G^{12}}$.

To compute $\widehat{m}$ we take transforms in the second equation in (1.4) to obtain

$$
\widehat{m}_{t}=\left(-\varepsilon|\xi|^{2} I-\eta \xi \xi^{t}\right) \widehat{m}-i c^{2} \hat{\rho} \xi
$$

To facilitate the computation, we introduce components parallel to and orthogonal to $\xi$. We thus write

$$
\widehat{m}(\xi, t)=a(\xi, t) \frac{\xi}{|\xi|}+b(\xi, t),
$$

where the vector $b$ is perpendicular to $\xi$, and where $a$ is the scalar $a=\widehat{m} \cdot \xi /|\xi|$. Substituting (3.6) into (3.5), we find that

$$
\left\{\begin{array}{l}
a_{t}=-\nu|\xi|^{2} a-i c^{2}|\xi| \hat{\rho} \\
b_{t}=-\varepsilon|\xi|^{2} b .
\end{array}\right.
$$


Thus

$$
b(\xi, t)=e^{-\varepsilon|\xi|^{2} t}\left(I-\frac{\xi \xi^{t}}{|\xi|^{2}}\right) \widehat{m}_{0}
$$

and

$$
a(\xi, t)=e^{-\nu|\xi|^{2} t}\left[a(\xi, 0)-i c^{2}|\xi| \int_{0}^{t} e^{\nu|\xi|^{2} s} \hat{\rho}(\xi, s) d s\right] .
$$

We substitute (3.4) into (3.9), perform the integration, and simplify, applying the relations $\lambda_{ \pm}+\nu|\xi|^{2}=-\lambda_{\mp}$ and $\lambda_{-} \lambda_{+}=c^{2}|\xi|^{2}$. The result is that

$$
a(\xi, t)=-i c^{2}|\xi|\left(\frac{e^{\lambda_{+} t}-e^{\lambda_{-} t}}{\lambda_{+}-\lambda_{-}}\right) \hat{\rho}_{0}+\left(\frac{\lambda_{+} e^{\lambda_{+} t}-\lambda_{-} e^{\lambda_{-} t}}{\lambda_{+}-\lambda_{-}}\right) \frac{\xi \cdot \widehat{m}_{0}}{|\xi|} .
$$

Substituting (3.8) and (3.10) into (3.6), we obtain finally that

$$
\begin{aligned}
& \widehat{m}(\xi, t)=-i c^{2}\left(\frac{e^{\lambda_{+} t}-e^{\lambda_{-} t}}{\lambda_{+}-\lambda_{-}}\right) \hat{\rho}_{0} \xi \\
& \quad+\left[e^{-\varepsilon|\xi|^{2} t} I+\left(\frac{\lambda_{+} e^{\lambda_{+} t}-\lambda_{-} e^{\lambda_{-} t}}{\lambda_{+}-\lambda_{-}}-e^{-\varepsilon|\xi|^{2} t}\right) \frac{\xi \xi^{t}}{|\xi|^{2}}\right] \widehat{m}_{0} .
\end{aligned}
$$

This determines $\widehat{G^{21}}$ and $\widehat{G^{22}}$.

In the following theorem we derive pointwise estimates for $\widehat{G}$ and its $\xi$ derivatives, as well as a series expansion for $\widehat{G}$ valid for large $|\xi|$.

Theorem 3.2. Let $G(x, t)$ be the Green's matrix of Lemma 3.1.

(a) Given $R>0$, there is a positive number $\vartheta$ such that, for multi-indices $\alpha$ and for $|\xi| \leq R$,

$$
\left|D_{\xi}^{\alpha} \widehat{G}(\xi, t)\right| \leq C e^{-\vartheta|\xi|^{2} t}(1+|\xi|)^{|\alpha|}(1+t)^{|\alpha|},
$$

where $C=C(R,|\alpha|)$.

(b) There is a positive constant $R$ such that the following series expansion is valid for $|\xi| \geq R$ : 


$$
\begin{aligned}
\widehat{G}(\xi, t)=e^{-\vartheta_{1}|\xi|^{2} t} \mathcal{M}_{1}+\cdots+e^{-\vartheta_{\ell}|\xi|^{2} t} \mathcal{M}_{\ell}+e^{-a t}\left[\begin{array}{ll}
1 & 0 \\
0 & 0
\end{array}\right] \\
+\left(\sum_{j=0}^{\infty}|\xi|^{-2 j-1} q_{j}(|\xi|, t)\right)\left[\begin{array}{cc}
0 & \frac{\xi^{t}}{|\xi|} \\
\frac{c^{2} \xi}{|\xi|} & 0
\end{array}\right] \\
+\left(\sum_{j=0}^{\infty}|\xi|^{-2 j-2} D_{j}(|\xi|, t)\right)\left[\begin{array}{cc}
1 & 0 \\
0 & \frac{\xi \xi^{t}}{|\xi|^{2}}
\end{array}\right]
\end{aligned}
$$

where $a=c^{2} / \nu, \vartheta_{k}$ are positive constants, $\mathcal{M}_{k}$ are matrices whose entries are linear combinations of constants and homogenous quadratic polynomials in $\xi /|\xi|, q_{j}$ are scalar functions, and $D_{j}$ are diagonal matrices. $q_{j}$ and $D_{j}$ satisfy the estimates

$$
\left|q_{j}(r, t)\right|,\left|D_{j}(r, t)\right| \leq C\left(e^{-a t / 2}+e^{-\nu r^{2} t / 2}\right) r_{0}^{2 j}
$$

for positive constants $C$ and $r_{0}$. Finally, there is a positive constant $\vartheta$ such that, for $|\xi| \geq R$ and $\alpha \neq 0$,

$$
\left|D_{\xi}^{\alpha} \widehat{G}(\xi, t)\right| \leq C(\alpha)\left(e^{-a t / 2}|\xi|^{-|\alpha|-1}+e^{-\vartheta|\xi|^{2} t}|\xi|^{-|\alpha|}\right) .
$$

Proof. We first prove the $\alpha=0$ case of (a), that is, that

$$
|\widehat{G}(\xi, t)| \leq C e^{-\vartheta|\xi|^{2} t}, \quad|\xi| \leq R .
$$

Observe from (3.2) that $\operatorname{Re}\left(\lambda_{-}\right) \leq-\frac{1}{2} \nu|\xi|^{2}$ always, and $\operatorname{Re}\left(\lambda_{+}\right) \leq-\frac{1}{2} \nu|\xi|^{2}$ when $|\xi| \leq 2 c / \nu$ and $\lambda_{+}$is complex. It is easy to check that there is also a positive constant $\vartheta$ depending on $R$ such that $\operatorname{Re}\left(\lambda_{+}\right) \leq-\vartheta|\xi|^{2}$ for $2 c / \nu<|\xi| \leq R$. Thus

$$
R e\left(\lambda_{ \pm}(\xi)\right) \leq-\vartheta|\xi|^{2}
$$

and therefore

$$
\left|e^{\lambda_{ \pm}(\xi) t}\right| \leq e^{-\vartheta|\xi|^{2} t}
$$

when $0 \leq|\xi| \leq R$. 
Now let $r=2 c / \nu$ be the resonant value of $|\xi|$, and choose a small positive number $\mu$. Then for $r+\mu \leq|\xi| \leq R$,

$$
\left|\lambda_{+}-\lambda_{-}\right|=\nu|\xi|^{2} \sqrt{1-r^{2}|\xi|^{-2}} \geq \nu|\xi|^{2} \sqrt{1-\left(\frac{r}{r+\mu}\right)^{2}} \geq C^{-1}
$$

and for $0 \leq|\xi| \leq r-\mu$,

$$
\left|\lambda_{+}-\lambda_{-}\right|=r c|\xi| \sqrt{1-\left(\frac{r-\mu}{r}\right)^{2}} \geq C^{-1}|\xi| .
$$

These bounds, together with (3.17), the fact that $\left|\lambda_{ \pm}\right| \leq C|\xi| \leq C$ for $|\xi| \leq R$, and the explicit representation (3.1), combine to prove (3.15) when $|\xi| \leq R$ and ||$\xi|-r| \geq \mu$.

To prove (3.15) when ||$\xi|-r| \leq \mu$, we apply (3.16) again to find that

$$
\begin{aligned}
\left|\frac{e^{\lambda_{+} t}-e^{\lambda_{-} t}}{\lambda_{+}-\lambda_{-}}\right| & \leq t \sup _{0 \leq s \leq 1} e^{t \operatorname{Re}\left(s \lambda_{-}+(1-s) \lambda_{+}\right)} \\
& \leq t e^{-\vartheta|\xi|^{2} t} \\
& \leq C e^{-\vartheta|\xi|^{2} t / 2}, \quad|| \xi|-r| \leq \eta .
\end{aligned}
$$

This establishes the required bounds for $\widehat{G^{12}}$ and $\widehat{G^{21}}$. For $\widehat{G^{11}}$ we simply observe that

$$
\begin{aligned}
\left|\widehat{G^{11}}(\xi)\right| & \leq\left|\lambda_{+} \frac{e^{\lambda_{+} t}-e^{\lambda_{-} t}}{\lambda_{+}-\lambda_{-}}\right|+\left|e^{\lambda_{+} t}\right| \\
& \leq C e^{-\vartheta|\xi|^{2} t}
\end{aligned}
$$

by (3.16) and (3.18). A similar argument applies to $\widehat{G^{22}}$. This completes the proof of (3.15).

The general statement (3.11) now follows easily by induction on $|\alpha|$. Since $\widehat{G}(\xi, t)=e^{-\left(i \xi_{j} A_{j}+\xi_{j} \xi_{k} B_{j k}\right) t}$ for appropriate matrices $A_{j}$ and $B_{j k}$, we may write

$$
\left\{\begin{array}{l}
\frac{\partial}{\partial t} \widehat{G}(\xi, t)=H(\xi) \widehat{G}(\xi, t) \\
\widehat{G}(\xi, 0)=I
\end{array}\right.
$$

where the entries of $H$ are quadratic polynomials in $\xi$. Thus for $\alpha \neq 0$,

$$
\left\{\begin{array}{l}
\frac{\partial}{\partial t} D_{\xi}^{\alpha} \widehat{G}=H D_{\xi}^{\alpha} \widehat{G}+\sum_{\substack{0<|\beta| \leq 2 \wedge|\alpha| \\
\beta+\gamma=\alpha}} \frac{\alpha !}{\beta ! \gamma !} D_{\xi}^{\beta} H D_{\xi}^{\gamma} \widehat{G} \\
D_{\xi}^{\alpha} \widehat{G}(\xi, 0)=0,
\end{array}\right.
$$


so that

$$
\begin{aligned}
\left|D_{\xi}^{\alpha} \widehat{G}(\xi, t)\right| & \leq \sum \frac{\alpha !}{\beta ! \gamma !} \int_{0}^{t}\left|\widehat{G}(\xi, t-s) D_{\xi}^{\beta} H(\xi) D_{\xi}^{\gamma} \widehat{G}(\xi, s)\right| d s \\
& \leq C(R,|\alpha|) \int_{0}^{t} e^{-\vartheta|\xi|^{2}(t-s)}\left[(1+|\xi|)(1+|\xi|)^{|\alpha|-1}(1+s)^{|\alpha|-1}\right. \\
& \left.+(1+|\xi|)^{|\alpha|-2}(1+s)^{|\alpha|-2}\right] e^{-\vartheta|\xi|^{2} s} d s \\
& \leq C e^{-\vartheta|\xi|^{2} t}(1+|\xi|)^{|\alpha|}(1+t)^{|\alpha|},
\end{aligned}
$$

as required. This completes the proof of (a).

To prove (b) we shall express $\widehat{G}$ in terms of certain fundamental solutions associated with the ode (3.3) satisfied by $\hat{\rho}(\xi, \cdot)$. These fundamental solutions admit contour integral representations, which are easily expanded and differentiated. Thus, let $p$ be the symbol

$$
p(z, r)=z^{2}+\nu r^{2} z+c^{2} r^{2},
$$

and observe that, by $(3.2)$, the roots $\lambda_{ \pm}(r)$ of $p(\cdot, r)$ satisfy

$$
\begin{aligned}
& \lambda_{+}(r)=-a+O\left(r^{-2}\right), \\
& \lambda_{-}(r)=-\nu r^{2}+a+O\left(r^{-2}\right)
\end{aligned}
$$

for large $r=|\xi|$. We define contours $\Gamma_{+}, \Gamma_{-}$, and $\Gamma_{0}$ in the complex plane to be the circles of radius $a / 2$ centered respectively at $-a,-\nu r^{2}+a$, and 0 , and we define

$$
\left\{\begin{array}{l}
E(r, t)=\frac{1}{2 \pi i} \int_{\Gamma_{-} \cup \Gamma_{+}} \frac{e^{t z}}{p(z, r)} d z \\
F(r, t)=E_{t}(r, t)+\nu r^{2} E(r, t) .
\end{array}\right.
$$

Then

$$
\left\{\begin{array}{l}
p\left(D_{t}, r\right) E=p\left(D_{t}, r\right) F=0 \\
E(r, 0)=0, \quad F(r, 0)=1, \\
E_{t}(r, 0)=1, \quad F_{t}(r, 0)=0
\end{array}\right.
$$

We shall also make use of the auxiliary function

$$
H(r, t)=e^{-\nu r^{2} t} \int_{0}^{t} e^{\nu r^{2} s} E(r, s) d s .
$$


By expanding $1 / p$ under the integral sign in powers of $r^{-1}$, we then obtain the following series expansions for large $r$ :

$$
\left\{\begin{array}{l}
E(r, t)=\sum_{j=0}^{\infty} E_{j}(r, t) r^{-2 j-2} \\
F(r, t)=e^{-a t}+\sum_{j=0}^{\infty} F_{j}(r, t) r^{-2 j-2} \\
H(r, t)=\sum_{j=0}^{\infty} H_{j}(r, t) r^{-2 j-4},
\end{array}\right.
$$

where

$$
\left|E_{j}(r, t)\right|,\left|F_{j}(r, t)\right|,\left|H_{j}(r, t)\right| \leq C\left(e^{-a t / 2}+e^{-\nu r^{2} t / 2}\right) r_{0}^{2 j}
$$

for suitable constants $C$ and $r_{0}$. For example, making the change of variable $w=z+\nu r^{2}-a$, we compute that

$$
\begin{aligned}
\int_{\Gamma_{-}(r)} \frac{e^{t z}}{p(z, r)} d z & =-e^{\left(a-\nu r^{2}\right) t} \int_{\Gamma_{0}} \frac{e^{t w}}{\nu r^{2} w}\left[1-\frac{(w+a)^{2}}{\nu r^{2} w}\right]^{-1} d w \\
& =\sum_{j=0}^{\infty} r^{-2 j-2}\left[-e^{\left(a-\nu r^{2}\right) t} \int_{\Gamma_{0}} \frac{(w+a)^{2 j}}{(\nu w)^{j+1}} e^{t w} d w\right]
\end{aligned}
$$

When $r$ is sufficiently large, the term in brackets here is bounded by

$$
C e^{\left(a-\nu r^{2}\right) t} e^{a t / 2} r_{0}^{2 j} \leq C e^{-\nu r^{2} t / 2} r_{0}^{2 j} .
$$

Expanding the integral over $\Gamma_{+}$in a similar way, we thus obtain the series (3.22) for $E$. The expansions for $F$ and $H$ then follow from the definitions (3.19) and (3.21).

We shall also require bounds for the $r$-derivatives of the fundamental solutions $E, F$, and $H$. For example, letting $b_{j}$ denote the contour integral on the right side of (3.24), we compute that

$$
\begin{aligned}
& \left(\frac{\partial}{\partial r}\right)^{k} \int_{\Gamma_{-}} \frac{e^{t z}}{p(z, r)} d z \\
& \quad=-e^{a t} \sum_{j=0}^{\infty} b_{j}(t) \sum_{\ell=0}^{k}\left(\begin{array}{l}
k \\
\ell
\end{array}\right)\left[\left(\frac{\partial}{\partial r}\right)^{\ell} e^{-\nu r^{2} t}\right]\left[\left(\frac{\partial}{\partial r}\right)^{k-\ell} r^{-2 j-2}\right] .
\end{aligned}
$$


The first brackets is bounded by $C r^{-\ell} e^{-2 \nu r^{2} t / 3}$ for large $r$, so that

$$
\begin{aligned}
& \left|\left(\frac{\partial}{\partial r}\right)^{k} \int_{\Gamma_{-}} \frac{e^{t z}}{p(z, r)} d z\right| \\
& \quad \leq C e^{\left(a-2 \nu r^{2} / 3\right) t} \sum_{j=0}^{\infty}\left|b_{j}(t)\right| r^{-2 j-2-k} \sum_{\ell=0}^{k}\left(\begin{array}{c}
k \\
\ell
\end{array}\right) \frac{(2 j+2) !}{(2 j+2-k+\ell) !} \\
& \quad \leq C(k) e^{-\nu r^{2} t / 2} r^{-k-2} .
\end{aligned}
$$

Applying similar estimates to the integral over $\Gamma_{+}$and passing these estimates through the definitions (3.19) and (3.21) of $F$ and $H$, we obtain that, for $k>0$,

$$
\begin{gathered}
\left|\frac{\partial^{k} E}{\partial r^{k}}\right|,\left|\frac{\partial^{k} F}{\partial r^{k}}\right| \leq C(k)\left(e^{-a t / 2}+e^{-\nu r^{2} t / 2}\right) r^{-k-2}, \\
\left|\frac{\partial^{k} H}{\partial r^{k}}\right| \leq C(k)\left(e^{-a t / 2}+e^{-\nu r^{2} t / 2}\right) r^{-k-4} .
\end{gathered}
$$

We now let $\hat{\rho}$ and $\widehat{m}$ be as in the proof of Lemma 3.1. $\hat{\rho}(\xi, \cdot)$ satisfies the initial-value problem (3.3), so that, by $(3.20)$,

$$
\hat{\rho}(\xi, t)=F(|\xi|, t) \hat{\rho}_{0}(\xi)+E(|\xi|, t)\left(-i \xi \cdot \widehat{m}_{0}(\xi)\right) .
$$

Writing

$$
\widehat{G}=\left[\begin{array}{ll}
\widehat{G}^{11} & \widehat{G}^{12} \\
\widehat{G}^{21} & \widehat{G}^{22}
\end{array}\right],
$$

where $\widehat{G}^{11}$ is a scalar and $\widehat{G}^{22}$ is $n \times n$, we then conclude that

$$
\widehat{G}^{11}(\xi, t)=F(|\xi|, t) \text { and } \widehat{G}^{12}(\xi, t)=-i E(|\xi|, t) \xi^{t} .
$$

(3.1) shows that $\widehat{G}^{21}=c^{2}\left(\widehat{G}^{12}\right)^{t}$, so that

$$
\widehat{G}^{21}(\xi, t)=-i c^{2} E(|\xi|, t) \xi .
$$

To represent $\widehat{G}^{22}$ in terms of the fundamental solutions, we again decompose $\widehat{m}$ into its components parallel to and orthogonal to $\xi$, as in (3.6). The orthogonal component $b(\xi, t)$ is given by (3.8), and to compute the radial component $a(\xi, t)$ we now substitute the representation (3.27) for $\hat{\rho}$ into the ode (3.7) and apply the definition (3.21) of $H$. The result is that, except for terms $e^{-\vartheta_{k}|\xi|^{2} t} \mathcal{M}_{k}$ as in (3.12),

$$
\widehat{G}^{22}(\xi, t)=-c^{2}|\xi|^{2} H(|\xi|, t) \frac{\xi \xi^{t}}{|\xi|^{2}} .
$$

Substituting the series (3.22) into the representations (3.28)-(3.30), we then obtain the required expansion (3.12) for $\widehat{G}$. The derivative estimates (3.14) follow in a straightforward way from $(3.25)-(3.26)$ by way of these same representations. 
4. Fourier multipliers. To derive a decomposition analogous to (1.15) from the estimates (3.11) and (3.12), we will need some results about $L^{p}$ multipliers. Most fundamental of these is the following classical result:

Proposition 4.1 (Marcinkiewic Multiplier Theorem). Let $\hat{f}(\xi) \in L^{\infty} \cap$ $C^{k}\left(\mathbb{R}^{n} /\{0\}\right)$, with $k=[n / 2]+1$, and

$$
\left|D_{\xi}^{\alpha} \hat{f}(\xi)\right| \leq C^{\prime}|\xi|^{-|\alpha|}, \quad|\alpha| \leq k .
$$

Then $\hat{f}(\xi)$ is an $L^{p}$ multiplier for $1<p<\infty$; that is, if we define $\widehat{f * g}=$ $(2 \pi)^{n / 2} \hat{f} \hat{g}$, then

$$
\|f * g\|_{p} \leq C_{p}\|g\|_{p} \text { for } g \in L^{2} \cap L^{p}, 1<p<\infty,
$$

where $C_{p}$ depends only on $p$ and the constant $C^{\prime}$, and is bounded on any closed interval $\left[p_{1}, p_{2}\right] \subset(1, \infty)$. (See Stein [18], p. 96.)

Typical examples of $L^{p}$ multipliers are given by the class of functions

$$
\hat{f}(\xi)=\chi(\xi) h\left(\frac{\xi}{|\xi|}\right)
$$

where $\chi$ is a $C^{\infty}$ cutoff function, constant except on a set bounded away from 0 and $\infty$, and $h \in C^{k}\left(S^{n}\right)$.

For our purposes, it is useful to sharpen Proposition 4.1 somewhat. The following two propositions, complementary to Proposition 4.1, turn out to be natural for the study of second-order hyperbolic-parabolic systems. with

$$
\left|D_{\xi}^{\alpha} \hat{f}(\xi)\right| \leq C^{\prime} \begin{cases}|\xi|^{-|\alpha|+\sigma_{1}} & |\xi| \leq R ;|\alpha|=n, \\ |\xi|^{-|\alpha|-\sigma_{2}} & |\xi| \geq R ;|\alpha|=n-1, n, n+1,\end{cases}
$$

where $\sigma_{1}, \sigma_{2}>0$ and

$$
n>2-2 \sigma_{2} .
$$

Then $\hat{f}(\xi)$ is continuous at 0 and $\infty$, and

$$
f=m_{1}+m_{2} \delta,
$$

where $m_{1} \in L^{1}\left(\mathbb{R}^{n}\right)$ satisfies $\left\|m_{1}\right\|_{1} \leq C\left(C^{\prime}\right), m_{2}$ is the constant

$$
m_{2}=(2 \pi)^{-n / 2} \lim _{|\xi| \rightarrow \infty} \hat{f}(\xi),
$$

and $\delta$ is the Dirac distribution. In particular, $\hat{f}(\xi)$ is a strong $L^{p}$ multiplier, $1 \leq p \leq \infty$, in the sense that, for any $g \in L^{p}$, 


$$
\|f * g\|_{p} \leq C\|g\|_{p}, 1 \leq p \leq \infty,
$$

where $C$ depends only on $\left|m_{2}\right| \leq\|\hat{f}\|_{\infty}$ and the constant $C^{\prime}$ above.

Proof. Set

$$
\begin{aligned}
& \hat{f}_{1}(\xi) \equiv \chi(\xi) \hat{f}(\xi), \\
& \hat{f}_{2}(\xi) \equiv(1-\chi(\xi)) \hat{f}(\xi),
\end{aligned}
$$

where

$$
\chi(\xi)= \begin{cases}1, & |\xi| \leq R-1, \\ 0, & |\xi| \geq R,\end{cases}
$$

is a smooth cutoff function, so that $f=f_{1}+f_{2}$.

Since $\chi \hat{f} \in L^{\infty}$, with compact support, the Hausdorff-Young inequality gives

$$
\left\|f_{1}\right\|_{\infty} \leq\left\|\widehat{f}_{1}\right\|_{1} \leq C
$$

so that $f_{1} \in L^{1}(|x| \leq R)$.

For $|\alpha|=n+1$, the Hausdorff-Young inequality gives

$$
\begin{aligned}
\left\|x^{\alpha} f_{2}\right\|_{\infty} & \leq\left\|\widehat{x^{\alpha} f_{2}}\right\|_{1} \\
& =\left\|D^{\alpha}(1-\chi) \hat{f}\right\|_{1} \\
& \leq C\left(\sum_{1 \leq|\beta| \leq|\alpha|}\left\|D_{\xi}^{\beta} \chi\right\|_{1}+\left\|(1-\chi) D_{\xi}^{\alpha} \hat{f}\right\|_{1}\right) \\
& \leq C\left(1+\left\|(1-\chi(\xi))|\xi|^{-(n+1)-\sigma_{2}}\right\|_{1}\right) \\
& \leq C .
\end{aligned}
$$

Thus

$$
\left|f_{2}(x)\right| \leq|x|^{-(n+1)}
$$

so that $f_{2} \in L^{1}(|x| \geq R)$.

Let $q_{1}=n /\left(n-\vartheta_{1} \sigma_{1}\right)$ and $q_{2}=n /\left(n-1+\vartheta_{2} \sigma_{2}\right)$, with $0<\vartheta_{j}<1$. Since $n+2 \sigma_{2}>2$, we can choose $\vartheta_{j}$ so that $1<q_{1}<2,1<q_{2} \leq 2$. Let $p_{1}, p_{2}$ be the Holder conjugates, $1 / q_{j}+1 / p_{j}=1$. Then, as above, for $|\alpha|=n$,

$$
\begin{aligned}
\left\|\chi^{\alpha} f_{1}\right\|_{p_{1}} & \leq\left\|D_{\xi}^{\alpha}(\chi \hat{f})\right\|_{q_{1}} \\
& \leq C\left(1+\left\|\chi(\xi)|\xi|^{-n+\sigma_{1}}\right\|_{q_{1}}\right) \\
& \leq C
\end{aligned}
$$


so that $|x|^{n} f_{1} \in L^{p_{1}}$. By Hölder's inequality,

$$
\begin{aligned}
\left\|f_{1}\right\|_{L^{1}(|x| \geq R)} & \leq\left\||x|^{n} f_{1}\right\|_{L^{p_{1}(|x| \geq R)}}\left\||x|^{-n}\right\|_{L^{q_{1}}\{|x| \geq R\}} \\
& \leq C,
\end{aligned}
$$

and $f_{1} \in L^{1}(|x| \geq R)$.

Similarly, $\left\|x^{\alpha} f_{2}\right\|_{p_{2}} \leq C$ for $|\alpha|=n-1$, so that $|x|^{n-1} f_{2} \in L^{p_{2}}$, and, by Hölder's inequality,

$$
\begin{aligned}
\left\||x|^{1-n}\left(|x|^{n-1} f_{2}\right)\right\|_{L^{1}(|x| \leq R)} & \leq\left\||x|^{n-1} f_{2}\right\|_{L^{p_{2}(|x| \leq R)}}\left\||x|^{1-n}\right\|_{L^{q_{2}(|x| \leq R)}} \\
& \leq C .
\end{aligned}
$$

Thus, the distribution $\tilde{f}_{2}(x) \stackrel{\text { def }}{=}|x|^{1-n}\left(|x|^{n-1} f_{2}(x)\right)$ is in $L^{1}(|x| \leq R)$. Since $\tilde{f}_{2} \equiv f_{2}$ away from the origin, $\tilde{f}_{2}$ is in fact in $L^{1}\left(\mathbb{R}^{n}\right)$.

Combining results, we find that

$$
m_{1}(x) \stackrel{\text { def }}{=} f_{1}(x)+\tilde{f}_{2}(x)
$$

is in $L^{1}$, with $\left\|m_{1}\right\|_{1} \leq C\left(C^{\prime}\right)$. On the other hand, the distribution $f-m_{1}=$ $f_{2}-\tilde{f}_{2}$ is supported at the point $x=0$, and its transform is bounded, since

$$
\left\|\hat{f}-\widehat{m}_{1}\right\|_{\infty} \leq\|\hat{f}\|_{\infty}+\left\|m_{1}\right\|_{1} .
$$

It follows (cf., e,g., [Donahue, p. 103]) that $f-m_{1}$ is a multiple of the delta distribution,

$$
f-m_{1}=m_{2} \delta
$$

verifying (4.6). Since $\widehat{m}_{1}$, as the transform of an $L^{1}$ function, is in $C_{0}\left(\mathbb{R}^{n}\right)$, it follows that $\hat{f}=\widehat{m}_{1}+(2 \pi)^{-n / 2} m_{2}$ is continuous at 0 and $\infty$, and that $\lim _{|\xi| \rightarrow \infty} \hat{f}(\xi)=(2 \pi)^{-n / 2} m_{2}$, as claimed.

Finally, for $g \in L^{p}, 1 \leq p<\infty$, we have

$$
\begin{aligned}
\|f * g\|_{p} & =\left\|\left(m_{1}+m_{2} \delta\right) * g\right\|_{p} \\
& \leq\left\|m_{1} * g\right\|_{p}+\left|m_{2}\right|\|g\|_{p} \\
& \leq\left\|m_{1}\right\|_{1}\|g\|_{p}+\left|m_{2}\right|\|g\|_{p} \\
& \leq\left(\frac{C}{2}+\left|m_{2}\right|\right)\|g\|_{p} \\
& \leq\left(\frac{C}{2}+\|\hat{f}\|_{\infty}\right)\|g\|_{p},
\end{aligned}
$$

proving (4.7).

Note that Proposition 4.2 is truly multi-dimensional in character, in the sense that the most interesting applications and also the strongest conclusions 
(see (4.5)) of the result are for the case $n \geq 2$. Another instance in which Proposition 4.1 can be sharpened is given in the following result:

Proposition 4.3. Let $\hat{g}(\xi, t)=\widehat{K}(\xi, t) \hat{f}(\xi)$, where $\widehat{K}(\xi, t)=e^{-\vartheta|\xi|^{2} t}, \hat{f}(\xi) \in$ $L^{\infty} \cap C^{n+1}\left(\mathbb{R}^{n}\right)$, and

$$
\left|D_{\xi}^{\beta} \hat{f}(\xi)\right| \leq C^{\prime}|\xi|^{-|\beta|}, \quad|\beta| \leq n+1
$$

Then $D_{x}^{\alpha} g(\cdot, t) \in L^{p}$ for $t>0$, for all $\alpha$, and for all $p \in[1, \infty]$, and

$$
\left\|D_{x}^{\alpha} g(\cdot, t)\right\|_{p} \leq C(|\alpha|) t^{(n / 2)(1 / p-1)-|\alpha| / 2} .
$$

In particular, $\left.\widehat{D_{x}^{\alpha} g(x, t}\right)=(i \xi)^{\alpha} \hat{g}(\xi, t)$ is a strong $L^{p}$ multiplier, with norm bounded by $C(|\alpha|, \vartheta) C^{\prime} t^{-|\alpha| / 2}$, where the constant $C(|\alpha|, \vartheta)$ depends only on $|\alpha|$ and $\vartheta$.

Proof. By Hausdorff-Young, for $|\beta|=n+1$,

$$
\begin{aligned}
& \left\|x^{\beta} D_{x}^{\alpha} g\right\|_{\infty} \leq C\left\|D_{\xi}^{\beta} \xi^{\alpha} \widehat{K} \hat{f}\right\|_{1} \\
& \quad \leq C \sum_{\beta=\left(\beta_{1}, \beta_{2}, \beta_{3}\right)}\left\||\xi|^{|\alpha|-\left|\beta_{1}\right|}\left|D_{\xi}^{\beta_{2}} \widehat{K}\right|\left|D_{\xi}^{\beta_{3}} \widehat{f}\right|\right\|_{1} \\
& \quad \leq C C^{\prime} \sum_{\beta}\left\||\xi|^{|\alpha|-\left|\beta_{1}\right|-\left|\beta_{3}\right|}\left(|\xi t|^{\left|\beta_{2}\right|}+|\xi|^{\left|\beta_{2}\right|-2} t^{\left|\beta_{2}\right|-1}+\cdots\right) e^{-\vartheta|\xi|^{2} t}\right\|_{1} \\
& \quad=C C^{\prime} t^{-n / 2-|\alpha| / 2+|\beta| / 2} .
\end{aligned}
$$

Thus

$$
\left|D_{x}^{\alpha} g\right| \leq C(|\alpha|) C^{\prime} t^{-n / 2-|\alpha| / 2}\left(\frac{|x|}{\sqrt{t}}\right)^{-(n+1)}
$$

for any $\alpha$, from which (4.18) follows, by scaling.

The following very relevant example distinguishes the classes of $L^{p}$ and strong $L^{p}$ multipliers.

Example 4.4. Let $\hat{f}(\xi)=P_{N}(\xi /|\xi|)$, with $P_{N}$ an $N$ th degree polynomial, and define

$$
\begin{aligned}
& \hat{f}_{1}(\xi)=\chi(\xi) \hat{f}(\xi), \\
& \hat{f}_{2}(\xi)=(1-\chi(\xi)) \hat{f}(\xi),
\end{aligned}
$$


where $\chi(\xi)$ is the cutoff function of (4.9) with support in $\{\xi:|\xi| \leq R\}$. Then each of $\hat{f}_{1}, \hat{f}_{2}, \hat{f}$ is an $L^{p}$ multiplier, $1<p<\infty$, but is not an $L^{1}$ or $L^{\infty}$ multiplier in the sense of (4.2).

Proof. We first observe that $f(x)$ has the simple form

$$
f(x)=P V q_{N}\left(\frac{x}{|x|}\right)|x|^{-n},
$$

where $q_{N}$ is a nontrivial $N$ th order polynomial. This follows by induction on monomials. For $N=1$, we have $\widehat{\xi}_{j} /|\xi|=C P V\left(x_{j} /|x|^{n+1}\right)$ by the Riesz formula [Stein]. For $N=2$,

$$
\frac{\widehat{\xi_{j} \xi_{k}}}{|\xi|^{2}}=D_{x_{j}} D_{x_{k}} \Delta^{-1} \delta=C D_{x_{j}} D_{x_{k}}|x|^{2-n}
$$

and we obtain (4.22) by direct calculation. We extend to $N>2$ by writing

$$
\frac{\xi_{j} \xi_{k}}{|\xi|^{2}} \cdot\left(\frac{\xi}{|\xi|}\right)^{\alpha}=C_{1} \xi_{j} D_{\xi_{k}}\left(\frac{\xi}{|\xi|}\right)^{\alpha}-C_{2} P_{|\alpha|}\left(\frac{\xi}{|\xi|}\right)
$$

whose Fourier transform, by induction, is

$$
\begin{gathered}
C_{1}^{\prime} D_{x_{j}}\left(x_{k} \widehat{\left(\frac{\xi}{|\xi|}\right)^{\alpha}}\right)-P V C_{2}^{\prime} q_{|\alpha|}\left(\frac{x}{|x|}\right)|x|^{-n} \\
=P V q_{|\alpha|+2}\left(\frac{x}{|x|}\right)|x|^{-n} .
\end{gathered}
$$

To see that $\hat{f}$ is not an $L^{\infty}$ multiplier, set

$$
g^{a}(x)=\left[1-\chi\left(\frac{x}{R a}\right)\right] \Gamma_{W}\left(\frac{x}{|x|}\right)
$$

where $\Gamma_{W}$ is the characteristic function of a set $W \subseteq S^{n-1}$ on which $\check{q_{N}}$ is positive. That is, $q_{N}(\vartheta) \geq C^{-1}$ for $-\vartheta \in W$. Then for $|x|<a / 2$,

$$
\begin{aligned}
f * g^{a}(x) & =P V \int_{0}^{\infty} r^{-1} \int_{|\vartheta|=1} q_{N}(\vartheta) \Gamma\left(\frac{x-r \vartheta}{|x-r \vartheta|}\right)\left[1-\chi\left(\frac{|x-r \vartheta|}{R a}\right)\right] d S_{\vartheta} d r \\
& =\int_{\frac{a}{2}}^{\infty} r^{-1} \int_{|\vartheta|=1} q_{N}(\vartheta) \Gamma\left(\frac{x-r \vartheta}{|x-r \vartheta|}\right)\left[1-\chi\left(\frac{|x-r \vartheta|}{R a}\right)\right] d S_{\vartheta} d r \\
& =+\infty
\end{aligned}
$$


since

$$
\begin{gathered}
\lim _{r \rightarrow+\infty} \int_{|\vartheta|=1} q_{N}(\vartheta)\left[1-\chi\left(\frac{|x-r \vartheta|}{R a}\right)\right] \Gamma\left(\frac{x-r \vartheta}{|x-r \vartheta|}\right) d S_{\vartheta} \\
=\int_{\vartheta \in W} q_{N}(-\vartheta) d S_{\vartheta} \geq C^{-1} .
\end{gathered}
$$

Thus, $f * g^{a}$ is not in $L^{\infty}$ for any $a>0$.

The same argument shows that $(1-\chi) f * g^{a}$ is not in $L^{\infty}$ for any $a>0$. Further, rescaling by $r^{\prime}=r / a$ shows that $\left\|\chi\left(f * g^{a}\right)\right\|_{\infty} \sim-\log a$ as $a \rightarrow 0$, so that neither $(1-\chi) f$ nor $\chi f$ satisfies (4.2) for $p=\infty$ with a constant independent of $a$. But, $\left\|f_{1}\right\|_{\infty} \leq\left\|\hat{f}_{1}\right\|_{1} \leq(R)^{n}$ implies that $f_{1} \in L^{1}(|x| \leq R)$, so that $\chi f_{1}$ satisfies (4.2). Similarly, $\left\||x|^{n+1} f_{2}(x)\right\|_{\infty} \leq\left\|D_{\xi}^{n+1} \hat{f}_{2}\right\|_{1} \leq C$, so that $f_{2} \in$ $L^{1}(|x| \geq R)$, and $(1-\chi) f_{2}$ satisfies (4.2). Thus, if either $f_{1}$ or $f_{2}$ satisfied (4.2), then either $\chi\left(f_{1}+f_{2}\right)=\chi f$ or $(1-\chi)\left(f_{1}+f_{2}\right)=(1-\chi) f$ would as well, a contradiction.

To see that $f$ is not an $L^{1}$ multiplier, let $g^{a}(x)=a^{-n} g(x / a)$ be a sequence of compactly supported test functions converging weakly to $\delta$. Then $\left(f * g^{a}\right)$ converges pointwise to $f$ as $a \rightarrow 0$, except at the point $x=0$, while $\left\|g^{a}\right\|_{1} \equiv 1$. Thus

$$
\lim _{a \rightarrow 0} \int_{\Omega}\left|f * g^{a}\right|=\int_{\Omega}|f|
$$

for any compact $\Omega \subset \mathbb{R}^{n} /\{0\}$. On the other hand, if $f$ were an $L^{1}$ multiplier, then there would be a constant $C$ such that

$$
C \geq\left\|f * g^{a}\right\|_{1} \geq \int_{\Omega}\left|f * g^{a}\right|
$$

and therefore that

$$
C \geq \int_{\Omega}|f|,
$$

contradicting (4.22). Clearly, the same reasoning holds for $\chi(x) f(x)$ and $(1-\chi(x)) f(x)$, so that, as above, neither $f_{1}(x)$ nor $f_{2}(x)$ satisfies (4.2).

5. A preliminary decay result. We are now ready to study the decay to equilibrium of solutions of the Navier-Stokes equations with real viscosity. We begin, in this section, by rederiving an $L^{p}, p \geq 2$, decay result of Kawashima [8], under weaker smoothness assumptions on the initial data. The decay rate we obtain for the solution is optimal only in $L^{2}$; we will improve it later on. 
Setting $u=\left[\begin{array}{c}\bar{\rho} \\ \bar{m}\end{array}\right]=\left[\begin{array}{c}\rho-\rho^{*} \\ m-m^{*}\end{array}\right]$, we can write the nonlinear system (1.1) in the form

$$
u_{t}-L u=\sum_{k} D_{x_{k}} Q^{k} ; u(x, 0)=u_{0}(x)
$$

where

$$
\begin{gathered}
L\left[\begin{array}{c}
\bar{\rho} \\
\bar{m}
\end{array}\right] \equiv\left[\begin{array}{c}
-\operatorname{div}(\bar{m}) \\
-c^{2} \nabla \bar{\rho}+\varepsilon \Delta \bar{m}+\eta \nabla \operatorname{div} \bar{m}
\end{array}\right], \\
Q^{k}=Q_{1}^{k}+Q_{2}^{k}=\left[\begin{array}{c}
0 \\
q_{1}^{k}(u)
\end{array}\right]+\left[\begin{array}{c}
0 \\
D_{x} q_{2}^{k}(u)
\end{array}\right],
\end{gathered}
$$

and

$$
q_{i}^{k}(u)=O\left(|u|^{2}\right)
$$

for $|u|$ sufficiently small.

Kawashima, [8], has proved the following basic existence result, using energy methods.

Proposition 5.1. Let $u_{0} \in H^{s+\ell}\left(\mathbb{R}^{n}\right), s=[n / 2]+1$, with $E \equiv\left\|u_{0}\right\|_{H^{s+\ell}}$ sufficiently small. Then, there exists a unique, global, classical solution $u \in H^{s+\ell}$ of (5.1), satisfying

$$
\left.\begin{array}{ll}
\left\|D_{x}^{\alpha} u\right\|_{2}, & 0 \leq|\alpha| \leq s+\ell \\
\left(\int_{0}^{\infty}\left\|D_{x}^{\alpha} u\right\|_{2} d t\right)^{1 / 2}, & 1 \leq|\alpha| \leq s+\ell \\
\left\|D_{x}^{\alpha} u\right\|_{\infty}, & 0 \leq|\alpha| \leq \ell
\end{array}\right\}=O(1) E,
$$

The solution operator $e^{L T}$ of the linear (left hand side) part of (5.1) can be expressed as

$$
e^{L t} u_{0}=G(t) * u_{0},
$$

where $G$ is the Green's function (more precisely, distribution) described in Section 3. Taking $D_{x}^{\alpha}$ of both sides of (5.1) and applying Duhamel's principle, we obtain the representation

$$
\begin{aligned}
D_{x}^{\alpha} u(t) & =G(t) * D_{x}^{\alpha} u_{0}+\int_{0}^{t} \sum_{k} D_{x_{k}} G(t-s) * D_{x}^{\alpha} Q^{k}(s) d s \\
& =I+I I,
\end{aligned}
$$

where we have suppressed the $x$ arguments for convenience. 
Lemma 5.2. Let

$$
\chi(\xi) \equiv \begin{cases}1 & |\xi| \leq R \\ 0 & |\xi| \geq R+1\end{cases}
$$

be a smooth cut off function, and decompose $G$ as $G=G_{1}+G_{2}$, where

$$
\begin{aligned}
\widehat{G}_{1}(\xi, t) & \equiv \chi(\xi) \widehat{G}(\xi, t), \\
\widehat{G}_{2}(\xi, t) & \equiv(1-\chi(\xi)) \widehat{G}(\xi, t) .
\end{aligned}
$$

Then:

(a) for any $R>0, D_{x}^{\alpha} G_{1} \in L^{p}$ for all $\alpha$, $p$, with

$$
\left\|D_{x}^{\alpha} G_{1}\right\|_{p} \leq C \begin{cases}t^{-n / 2(1-1 / p)-|\alpha| / 2} & t \geq 1, p \geq 2 \\ 1 & t \leq 1, \text { all } p\end{cases}
$$

(b) for $R$ sufficiently large, there is $b>0$ for which

$$
\widehat{G}_{2}=e^{-b t} M(\xi, t)
$$

and

$$
\widehat{D_{x_{j}} G_{2}} \cdot\left[\begin{array}{cc}
0 & 0 \\
0 & I
\end{array}\right]=e^{-b t}\left(1+t^{-1 / 2}\right) \mathcal{M}(\xi, t)
$$

where $M$ and $\mathcal{M}$ are, respectively, strong $(1 \leq p \leq \infty)$ and ordinary $(1<$ $p<\infty) L^{p}$ multipliers, with norms bounded by $C$ and $C(p)$ independent of time.

Proof. Let $p, q$ denote Hölder conjugates, $1 / p+1 / q=1$.

(a) The estimates in Lemma 3.2(a), together with the fact that $D_{\xi}^{\beta} \chi(\xi)$ is supported on $\{R \leq|\xi| \leq R+1\}$, for $|\beta|>0$, give

$$
\left|D_{\xi}^{\beta}\left(\xi^{\alpha} \widehat{G}_{1}\right)\right| \leq C|\xi|^{|\alpha|}(1+|\xi|)^{|\beta|}(1+t)^{|\beta|} e^{-\vartheta|\xi|^{2} t},
$$


for some $\vartheta>0$. Taking $|\beta|=0$, we find that $\xi^{\alpha} \widehat{G}_{1}$ is Schwartz class, so that $D_{x}^{\alpha} G_{1} \in L^{p}$ for all $p$. For $p \geq 2$, the Hausdorff-Young inequality gives

$$
\begin{aligned}
\left\|D_{x}^{\alpha} G_{1}(x, t)\right\|_{p} & \leq\left\|\xi^{\alpha} \widehat{G}_{1}(\xi, t)\right\|_{q} \\
& \leq\left(\int_{|\xi| \leq R}|\xi|^{|\alpha| q} e^{-\vartheta|\xi|^{2} t} d \xi\right)^{1 / q} \\
& \leq C \begin{cases}t^{-n / 2(1-1 / p)-|\alpha| / 2} & t \geq 1 \\
1 & t \leq 1 .\end{cases}
\end{aligned}
$$

For $t \leq 1,(5.13)$ gives

$$
\left|D_{\xi}^{\beta}\left(\xi^{\alpha} \widehat{G}_{1}\right)\right| \leq C^{\prime} \begin{cases}1 & |\xi| \leq R+1 \\ 0 & |\xi| \geq R+1\end{cases}
$$

Thus, Proposition 4.2 implies that

$$
\left\|D_{x}^{\alpha} G_{1}\right\|_{1} \leq C .
$$

Interpolating between (5.14) and (5.16), we obtain

$$
\left\|D_{x}^{\alpha} G_{1}\right\|_{p} \leq C, \quad t \leq 1, \text { all } p,
$$

completing the proof of (a).

(b) The estimates of Lemma 3.2(b) give

$$
D_{\xi}^{\beta} \widehat{G}_{2}(\xi, t) \leq C \begin{cases}0 & |\xi| \leq R \\ |\xi|^{-|\beta|}\left(e^{-a t / 2}|\xi|^{-1}+e^{-\vartheta|\xi|^{2} t}\right) & |\xi| \geq R .\end{cases}
$$

Applying Proposition 4.1, we have

$$
\widehat{G}_{2}(x, t)=e^{-b t} M,
$$

where $b<\min \left\{a / 2, \vartheta R^{2}\right\}$ and $M$ is an $L^{p}$ multiplier with norm bounded by $C_{p}$ independent of $t$.

To see that $M$ is in fact a strong $L^{p}$ multiplier, we must look more closely. Setting

$$
\hat{g}_{2}(\xi, t)=\widehat{G}_{2}(\xi, t)-(1-\chi(\xi)) \sum \hat{K}_{\ell} \mathcal{M}_{\ell}
$$


where $\hat{K}_{\ell}, \mathcal{M}_{\ell}$ are as in (3.12), we have

$$
D_{\xi}^{\beta} \hat{g}_{2}(\xi, t) \leq C \begin{cases}0 & |\xi| \leq R, \\ |\xi|^{-|\beta|-1} e^{-b t} & |\xi| \geq R,|\beta|>0 .\end{cases}
$$

Applying Proposition 4.2, we find that $\hat{g}_{2}(x, t)=e^{-b t} M(\xi, t), M$ a strong $L^{p}$ multiplier. More precisely, we have

$$
g_{2}(x, t)=e^{-a t} \delta\left(\begin{array}{ll}
1 & 0 \\
0 & 0
\end{array}\right)+e^{-b t} m(x, t)
$$

where $m(\cdot, t) \in L^{1},\|m(\cdot, t)\|_{1} \leq C$, and $a=c^{2} / \nu=c^{2} /(\varepsilon+\eta)$.

The remaining term, $(1-\chi)\left(\sum \hat{K}_{\ell} \mathcal{M}_{\ell}(\xi)\right)$, has Fourier transform in $L^{p}$ for all $p$, with

$$
\left\|D_{x}^{\alpha} \widehat{\sum \hat{K}_{\ell} \mathcal{M}_{\ell}}(\xi)\right\|_{1} \leq C e^{-b t} t^{-|\alpha| / 2}, \quad b>0
$$

For, in the factorization

$$
\begin{aligned}
(1-\chi(\xi)) & e^{-\vartheta_{\ell}|\xi|^{2} t} \mathcal{M}\left(\frac{\xi}{|\xi|}\right) \\
& =(1-\chi(\xi)) e^{-(1 / 2) \vartheta_{\ell}|\xi|^{2} t} \mathcal{M}\left(\frac{\xi}{|\xi|}\right) \times e^{-(1 / 2) \vartheta(\xi)^{2} t}
\end{aligned}
$$

the first term satisfies the hypotheses of Proposition 4.1 with constant $C^{\prime}(t) \leq$ $C e^{-(1 / 2) \vartheta_{\ell} R^{2} t}$, and the second is the transform of the heat kernel. Applying Proposition 4.3, we obtain (5.23), with $b<\min _{\ell}\left\{\frac{1}{2} \vartheta_{\ell} R^{2}\right\}$. This completes the proof of (5.11).

From Lemma 3.2(b), we have, for $|\beta| \geq 0$,

$$
\left|D_{\xi}^{\beta} \hat{g}_{2}(\xi) \cdot\left(\begin{array}{ll}
0 & 0 \\
0 & I
\end{array}\right)\right| \leq C \begin{cases}0 & |\xi| \leq R \\
|\xi|^{-|\beta|-1} e^{-b t} & |\xi| \geq R .\end{cases}
$$

With Proposition 4.1, this implies that

$$
\widehat{D_{x_{j}} g_{2}(x, t)}=e^{-b t} \mathcal{M}^{\prime}(\xi, t),
$$

with $\mathcal{M}^{\prime}$ an $L^{p}$ multiplier uniformly bounded in time. With (5.23), $|\alpha|=1$, this gives (5.12), and we are done. 
Remark. More precisely, we have

$$
M=\left[\left(\begin{array}{ll}
1 & 0 \\
0 & 0
\end{array}\right)\right] \delta(x)+m(x)
$$

where

$$
|m(x)| \leq \begin{cases}C|x|^{-n+1} & |x| \leq R \\ C(N)|x|^{-N} & |x|>R\end{cases}
$$

for any integer $N$. The first fact follows by the expansion (3.12), the second from the proof of Lemma 4.2 .

Remark. Example 4.4 shows that $\mathcal{M}$ is definitely not a strong $L^{p}$ multiplier. For, the $(1,1+k)$ entry of $\xi_{j} \widehat{G}_{2}(\xi, t)$ can be expressed more precisely as

$$
e^{-b t}(1-\chi(\xi))\left[\frac{\xi_{j} \xi_{k}}{|\xi|^{2}}+O\left(\frac{1}{|\xi|}\right) \mathcal{M}^{\prime}\right] \text {. }
$$

By Proposition 4.2, the term $O(1 /|\xi|)(1-\chi(\xi)) \mathcal{M}^{\prime}$ is a strong $L^{p}$ multiplier, while, by Example $4.4,(1-\chi(\xi))\left(\xi_{j} \xi_{k} /|\xi|^{2}\right)$ is not.

Theorem 5.3. Let $u_{0} \in H^{s+\ell} \cap L^{1}\left(\mathbb{R}^{n}\right)$, where $s=[n / 2]+1$ and $\ell$ is a positive integer. If

$$
E \stackrel{\text { def }}{=} \max \left\{\left\|u_{0}\right\|_{H^{s+\ell}},\left\|u_{0}\right\|_{1}\right\}
$$

is sufficiently small, then the solution $u(\cdot, t)$ of (5.1) satisfies

$$
\begin{aligned}
& \left\|D_{x}^{\alpha} u\right\|_{p} \leq E\left\{\begin{array}{c}
C(|\alpha|)(1+t)^{-n / 2(1-1 / p)-1 / 2 \min \{|\alpha|, \ell-2-|\alpha|\}} \\
\text { for }|\alpha| \leq \ell-2,2 \leq p \leq \infty, \ell \geq 3 \\
C(|\alpha|, p)(1+t)^{-n / 2(1-1 / p)-1 / 2\{\min |\alpha|, \ell-1-|\alpha|\}} \\
\text { for }|\alpha| \leq \ell-1,2 \leq p<\infty,
\end{array}\right. \\
& \left\|D_{x}^{\alpha}\left(u-e^{L t} u_{0}\right)\right\|_{p} \\
& \leq E^{2} L(t)\left\{\begin{array}{c}
C(|\alpha|)(1+t)^{-n / 2(1-1 / p)-1 / 2 \min \{|\alpha|,(\ell-3-|\alpha|)\}-1 / 2} \\
\text { for }|\alpha| \leq \ell-2,2 \leq p \leq \infty, \ell \geq 3 \\
C(|\alpha|, p)(1+t)^{-n / 2(1-1 / p)-1 / 2 \min \{|\alpha|,(\ell-1-|\alpha|)\}-1 / 2} \\
\text { for }|\alpha| \leq \ell-1,2 \leq p<\infty,
\end{array}\right.
\end{aligned}
$$

where $e^{L t}$ is as in (5.6) and $L(t)=\log (1+t)$ if $n=2, L(t)=1$ otherwise. 
Proof. We first prove the estimates (5.28) and (5.29) for $3 \leq|\alpha| \leq \ell-2$. Set

$$
\begin{aligned}
& A(t) \stackrel{\text { def }}{=} \sup _{\substack{0 \leq s \leq t \\
2 \leq p \leq \infty \\
|\alpha| \leq \ell-2}}(1+s)^{n / 2(1-1 / p)+1 / 2 \min \{|\alpha|, \ell-2-|\alpha|\}}\left\|D_{x}^{\alpha} u(\cdot, s)\right\|_{p}, \\
& B(t) \stackrel{\text { def }}{=} \sup _{\substack{0 \leq s \leq t \\
2 \leq p \leq \infty \\
|\alpha| \leq \ell-2}} L(t)^{-1}(1+s)^{n / 2(1-1 / p)+1 / 2 \min \{|\alpha|, \ell-3-|\alpha|\}+1 / 2} \\
& \times\left\|D_{x}^{\alpha}\left(u(\cdot, s)-e^{L s} u_{0}\right)\right\|_{p}
\end{aligned}
$$

Claim. For E sufficiently small,

$$
\begin{gathered}
A(t) \leq C\left(E+A(t)^{2}+A(t)^{\ell-2}\right), \quad t \geq 0 \\
B(t) \leq C\left(E^{2}+A(t)^{2}+A(t)^{\ell-2}\right), \quad t \geq 0 .
\end{gathered}
$$

Assuming (5.32)-(5.33), we obtain (5.28)-(5.29) by continuous induction. For, rearranging (5.32) gives

$$
A(t) \leq \frac{C E}{1-C\left(A(t)+A(t)^{\ell-2}\right)}
$$

Taking $4 C^{2} E<\frac{1}{2}$, we obtain $A(t) \leq 2 C E$ for all $t \geq 0$, using continuity of $A(t)$, (5.34), and induction. This proves (5.28) and, together with (5.33), (5.29), for $3 \leq|\alpha| \leq \ell-2$.

Proof of Claim. Let $|\alpha| \leq \ell-2$. By Proposition 5.1 and interpolation, we have

$$
\left.\begin{array}{l}
\left\|D_{x}^{\beta} u\right\|_{p}, 2 \leq p \leq \infty \\
\left(\int_{0}^{t}\left\|D_{x}^{\beta} u\right\|_{2}^{2}(s) d s\right)^{1 / 2}
\end{array}\right\} \leq C E \quad \text { for }|\beta| \leq|\alpha|+2 .
$$

Likewise, from (5.3)-(5.4), we have, for $2 \leq p \leq \infty$,

$$
\left\|D_{x}^{\alpha} Q\right\|_{p} \leq C E^{2}
$$




$$
\begin{aligned}
\int_{0}^{t}\|Q\|_{1} d s & \leq C \int_{0}^{t}\left\||u|^{2}+|u|\left|D_{x} u\right|\right\|_{1} d s \\
& \leq C \int_{0}^{t}\left(\|u\|_{2}^{2}+\left\|D_{x} u\right\|_{2}^{2}\right) d s \\
& \leq C \int_{0}^{t}\left(A(s)^{2}(1+s)^{-n / 2}+\left\|D_{x} u\right\|_{2}^{2}\right) d s \\
& \leq C\left(E^{2}+A(t)^{2}\right) \begin{cases}\log (1+t) & n=2 \\
1 & n \geq 3,\end{cases}
\end{aligned}
$$

$$
\begin{gathered}
\left.\begin{array}{l}
\left\|D_{x}^{\alpha} Q_{1}\right\|_{2 p /(p+2)} \\
\left\|D_{x}^{\beta} Q_{2}\right\|_{2 p /(p+2)},|\beta|=|\alpha|-1
\end{array}\right\} \\
\leq C \sup _{\sum\left|\gamma_{j}\right|=|\alpha|}\left\|D_{x}^{\gamma_{1}} u\right\|_{p}\left\|D_{x}^{\gamma_{2}} u\right\|_{2} \Pi_{j \geq 3}\left\|D_{x}^{\gamma_{j}} u\right\|_{\infty} \\
\leq C\left(A(t)^{2}+A(t)^{|\alpha|}\right)(1+t)^{-n / 2(1-1 / p)-1 / 2 \min \{|\alpha|, \ell-2-|\alpha|\}-n / 4},
\end{gathered}
$$

and the critical estimate,

$$
\begin{array}{cl} 
& \left\|D_{x} D_{x}^{\alpha} Q_{j}\right\|_{p} \leq \\
\leq C \sup _{\sum\left|\gamma_{j}\right|=|\alpha|+j}\left\|D_{x}^{\gamma_{1}} u\right\|_{p} \prod_{j \geq 2}\left\|D_{x}^{\gamma_{j}} u\right\|_{\infty} & \\
\leq A(t)\left(E+A(t)^{2}+A(t)^{|\alpha|}\right)(1+t)^{-n / 2(1-1 / p)-1 / 2 \min \{|\alpha|, \ell-3-|\alpha|\}-1 / 2}, & |\alpha| \leq \ell-2 .
\end{array}
$$

(We use the condition $\ell \geq 3$ precisely in (5.39), to bound the term $\left\|D_{x} D^{\alpha} u\right\|_{p}\left\|D_{x} u\right\|_{\infty}$ occurring for $|\alpha|=\ell-3$.)

Let $I$ and $I I$ be as in (5.7) and $2 \leq p \leq \infty$. Applying Lemma 5.2, we have

$$
\begin{aligned}
\|I\|_{p} & =\left\|D^{\alpha}\left(G * u_{0}\right)\right\|_{p} \\
& \leq\left\|D_{x}^{\alpha} G_{1} * u_{0}\right\|_{p}+\left\|G_{2} * D_{x}^{\alpha} u_{0}\right\|_{p} \\
& \leq\left\|D_{x}^{\alpha} G_{1}\right\|_{p}\left\|u_{0}\right\|_{1}+e^{-b t}\left\|D_{x}^{\alpha} u_{0}\right\|_{p} \\
& \leq C E(1+t)^{-n / 2(1-1 / p)-|\alpha| / 2} .
\end{aligned}
$$

Likewise, combining (5.36)-(5.39) with Lemma 5.2, we have for $|\alpha|>0$ and $|\beta|=|\alpha|-1$, 


$$
\begin{aligned}
\|I I\|_{p}= & \left\|\int_{0}^{t} D_{x} G(t-s) * D_{x}^{\alpha} Q(s) d s\right\|_{p} \\
\leq & \int_{0}^{t / 2}\left\|D_{x}^{\alpha} D_{x} G_{1} * Q\right\|_{p}+\int_{t / 2}^{t}\left\|D_{x} G_{1} * D_{x}^{\alpha} Q_{1}\right\|_{p} \\
& \quad+\int_{t / 2}^{t}\left\|D_{x}^{2} G_{1} * D_{x}^{\beta} Q_{2}\right\|_{p}+\int_{0}^{t}\left\|G_{2} * D_{x} D_{x}^{\alpha} Q\right\|_{p} \\
\leq & \int_{0}^{t / 2}\left\|D_{x}^{\alpha} D_{x} G_{1}\right\|_{p}\|Q\|_{1}+\int_{t / 2}^{t}\left\|D_{x} G_{1}\right\|_{2}\left\|D_{x}^{\alpha} Q_{1}\right\|_{2 p /(p+2)} \\
& +\int_{t / 2}^{t}\left\|D_{x}^{2} G_{1}\right\|_{2}\left\|D_{x}^{\beta} Q_{2}\right\|_{2 p /(p+2)}+\int_{0}^{t} e^{-b(t-s)}\left\|D_{x} D_{x}^{\alpha} Q\right\|_{p} \\
\leq & C(1+t)^{-n / 2(1-1 / p)-|\alpha| / 2-1 / 2} \int_{0}^{t / 2}\|Q(s)\|_{1} d s \\
& +C\left(A(t)^{2}+A(t)^{|\alpha|}\right)(1+t)^{-n / 2(1-1 / p)-1 / 2 \min \{|\alpha|, \ell-2-|\alpha|\}-n / 4} \\
& +C A(t)\left(E+A(t)+A(t)^{|\alpha|}\right) \int_{0}^{t} e^{-b(t-s)} \\
& \times(1+s)^{-n / 2(1-1 / p)-1 / 2 \min \{|\alpha|, \ell-3-|\alpha|\}-1 / 2} d s .
\end{aligned}
$$

In the case $|\alpha|=0$, we can replace $\int_{t / 2}^{t}\left\|D_{x}^{2} G_{1} * D_{x}^{\beta} Q_{2}\right\|_{p}$ with $\int_{t / 2}^{t} \| D_{x} G_{1} *$ $D_{x}^{\alpha} Q_{2} \|_{p}$ in the second line of (5.41) and obtain the same result. We therefore have

$$
\begin{aligned}
\|I I\|_{p} & \leq C\left(E^{2}+A(t)^{2}+A(t)^{|\alpha|}\right) \\
& \times(1+t)^{-n / 2(1-1 / p)-1 / 2 \min \{|\alpha|, \ell-3-|\alpha|\}-1 / 2} \quad \begin{cases}\log t, & n=2 \\
1, & n \geq 3 .\end{cases}
\end{aligned}
$$

Combining (5.40)-(5.42) with

$$
\begin{aligned}
& D_{x}^{\alpha} u=I+I I, \\
& D_{x}^{\alpha}\left(u-e^{L t} u_{0}\right)=I I,
\end{aligned}
$$

we obtain (5.32)-(5.33), and we are done.

Finally, the case that $|\alpha|=\ell-1$ or $|\alpha| \leq 2$ follows by a similar argument, with

$$
\begin{aligned}
& A(t) \stackrel{\text { def }}{=} \sup \left\{(1+s)^{n / 2(1-1 / p)+1 / 2 \min \{|\alpha|, \ell-1-|\alpha|\}}\left\|D_{x}^{\alpha} u(\cdot, s)\right\|_{p}\right\}, \\
& B(t) \stackrel{\text { def }}{=} \sup \left\{(1+s)^{n / 2(1-1 / p)+1 / 2 \min \{|\alpha|, \ell-1-|\alpha|\}}\left\|D_{x}^{\alpha}\left(u-e^{L t} u_{0}\right)\right\|_{p}\right\},
\end{aligned}
$$


by substituting

$$
\int D_{x} G_{2}\left[\begin{array}{cc}
0 & 0 \\
0 & I
\end{array}\right] * D_{x}^{\alpha} Q
$$

for

$$
\int G_{2} * D_{x} D_{x}^{\alpha} Q
$$

in (5.42), and replacing (5.39) by the bound

$$
\begin{aligned}
\left\|D_{x}^{\alpha} Q\right\|_{p} \leq A(t) & \left(E+A(t)^{2}+A(t)^{|\alpha|}\right) \\
& \times(1+t)^{-n / 2(1-1 / p)-1 / 2 \min \{|\alpha|, \ell-1-|\alpha|\}-1 / 2} .
\end{aligned}
$$

Since $D_{x} G_{2}\left[\begin{array}{cc}0 & 0 \\ 0 & I\end{array}\right]$ is only an $L^{p}$ multiplier for $2 \leq p<\infty$, the bound $C(|\alpha|)$ must be replaced by $C(|\alpha|, p)$.

Remark. The results of Theorem 5.3 can be established in $L^{2}$ without resort to Proposition 4.1 and Lemma 5.2 , since $\mathcal{M}$ is trivially an $L^{2}$ multiplier. With enough $([n / 2]+1)$ extra smoothness on the initial data, the results for $L^{p}$, $p \geq 2$ then follow by Sobolev bounds. However, the decomposition of the Green's function obtained in Lemma 5.2 via Proposition 4.1 allows us to obtain the results for $p>2$ with far less smoothness assumed on the initial data. Further, Lemma 5.2 will be essential in our analysis, in Sections 7 and 8 , of the case $p<2$.

6. Effective artificial viscosity and $L^{p}$ decay to diffusion waves, $p \geq 2$. We continue the study of the large-time behavior of the solution $u(\cdot, t)$ of the nonlinear Navier-Stokes system (1.1) in $L^{p}$ for $p \in[2, \infty]$. As we saw in Theorem $5.3, u(\cdot, t)$ is well-approximated for large time by $G(\cdot, t) * u_{0}$, where $G$ is the Green's matrix for the linearized Navier-Stokes system (1.4). While this result clarifies in a significant way the asymptotic behavior of $u$, it raises the issue of the "shape" of $G(\cdot, t) * u_{0}$ itself for large time. Observe that the linearized system (1.4) is not strictly parabolic, nor does the symbol associated with its first-order convection terms commute with that of its second order diffusion terms. As we shall see in Theorem 6.2 below, however, the matrices $B_{j k}$ occurring in (1.4) (in the notation of $\S 2$ ) may be replaced by artificial viscosity matrices $\tilde{B}_{j k}$, in such a way that the resulting system does become strictly parabolic, the symbols $\xi_{j} A_{j}$ and $\xi_{j} \xi_{k} \tilde{B}_{j k}$ do commute, and, most important, the solution $u(\cdot, t)$ of the nonlinear Navier-Stokes system (1.1) is just as well approximated by $\tilde{G}(\cdot, t) * u_{0}$ as by $G(\cdot, t) * u_{0}$, in the sense of Theorem 5.3. The aforementioned commutativity implies that $\tilde{G}(\cdot, t)$ is simply the convolution of two fundamental solutions - one corresponding to the hyperbolic part (linearized Euler equations), 
and the other to an explicit parabolic operator canonically associated to the Navier-Stokes system. These results enable us to greatly refine the description given in Theorem 5.3 of the asymptotic behavior of $u$. In addition, the strict parabolicity of the artificial viscosity system enables us to show that, when $u_{0}$ has a first moment, the corresponding solution $u(\cdot, t)$ to the nonlinear NavierStokes system is asymptotic to the convecting diffusion wave $\tilde{G}(\cdot, t) U$, where $U$ is the total mass of $u_{0}$. These results, stated in Theorems 6.7 and 6.8 below, represent the culmination of our analysis of solutions of the Navier-Stokes system in $L^{p}$ for $p \in[2, \infty]$.

We begin with a brief discussion of artificial viscosity in a rather general setting. Thus consider the following pde for an unknown vector function $u$ : $\mathbb{R}^{n} \times[0, \infty) \rightarrow \mathbb{R}^{m}:$

$$
u_{t}+A_{j} u_{x_{j}}=B_{j k} u_{x_{j} x_{k}}
$$

where $A_{j}$ and $B_{j k}$ are constant, real $m \times m$ matrices. If the differential operator in (6.1) satisfies the Hadamard-Petrovsky condition, then there will be a Green's matrix $G \in C^{\infty}\left([0, \infty) ; \mathcal{S}^{\prime}\left(\mathbb{R}^{n}\right)\right)$ : the columns of $G$ are solutions of $(6.1)$, and $G(0)=\delta I$. In addition, if (6.1) is dissipative in a suitable sense, then, as we proved in Section 5 for the linearized Navier-Stokes system and in Section 2 for more general linear systems,

$$
\left\|G(\cdot, t) * u_{0}\right\|_{L^{2}} \leq C\left(u_{0}\right) t^{-n / 4}
$$

for $u_{0}$ in a fairly broad class. We seek to replace the system (6.1) by a system which enjoys the special properties discussed above, but without altering the asymptotic character of its solutions in an essential way.

Definition. Let $G$ be the Green's matrix for (6.1) as described above, and assume that there is a symmetric, positive definite matrix $P$ such $P A_{j}$ is symmetric for each $j$, as in Section 2. An effective artificial viscosity for (6.1) is then a measurable mapping $\xi \mapsto \tilde{B}(\xi)$ from $\mathbb{R}^{n}$ into the set of $m \times m$ matrices such that

$$
\tilde{B}(\xi)=|\xi|^{2} \tilde{B}\left(\frac{\xi}{|\xi|}\right), \xi \in \mathbb{R}^{n}
$$

$$
\tilde{B}(\xi) \text { and } \xi_{j} A_{j} \text { commute, } \xi \in \mathbb{R}^{n}
$$


there is a positive constant $\varepsilon$ such that

$$
\begin{gathered}
\langle P \tilde{B}(\xi) w, w\rangle_{\mathbb{R}^{m}} \geq \varepsilon|\xi|^{2}|w|^{2}, \\
\xi \in \mathbb{R}^{n}, w \in \mathbb{R}^{m} ; \\
t^{n / 4}\left\|[G(\cdot, t)-\tilde{G}(\cdot, t)] * u_{0}\right\|_{L^{2}} \rightarrow 0 \text { as } t \rightarrow \infty
\end{gathered}
$$

for all $u_{0} \in L^{1} \cap L^{2}$, where $\hat{\tilde{G}}(\cdot, t)=\exp \left(-i \xi_{j} A_{j} t\right) \exp (-\tilde{B}(\xi) t)$.

Though we do not prove it here, there exists an effective artificial viscosity for any hyperbolic-parabolic system which is stable in the sense of Kawashima [8]. We shall demonstrate the existence of an effective artificial viscosity for the Navier-Stokes system by explicit computation; the procedure in the general case is similar (see (6.9) below). Uniqueness is quite straightforward:

Proposition 6.1. Assume that there is a symmetric, positive definite matrix $P$ such that $P A_{j}$ is symmetric for each $j$, and let $\tilde{B}_{1}$ and $\tilde{B}_{2}$ be effective artificial viscosities for the system (6.1). Then $\tilde{B}_{1}=\tilde{B}_{2}$.

Proof. Fix $\bar{u} \in \mathbb{R}^{m}$ and define $u_{0} \in L^{1} \cap L^{2}$ by $\hat{u}_{0}(\xi)=e^{-|\xi|^{2}} \bar{u}$. Letting $\tilde{G}_{1}$ and $\tilde{G}_{2}$ be the corresponding Green's matrices $\hat{\tilde{G}}_{\ell}(\xi, t)=\exp \left[\left(-i \xi_{j} A_{j}-\tilde{B}_{\ell}(\xi)\right) t\right]$, we then find from (6.3), (6.4), and the symmetrizability that

$$
\begin{aligned}
t^{n / 2} \| & {\left[G_{2}(\cdot, t)-G_{1}(\cdot, t)\right] * u_{0} \|_{2}^{2} } \\
& =t^{n / 2} \int\left|e^{-i \xi_{j} A_{j} t}\left[e^{-\tilde{B}_{2}(\xi) t}-e^{-\tilde{B}_{1}(\xi) t}\right] \bar{u}\right|^{2} e^{-2|\xi|^{2}} d \xi \\
& \geq C^{-1} \int\left|\left[e^{-\tilde{B}_{2}(y)}-e^{-\tilde{B}_{1}(y)}\right] \bar{u}\right|^{2} e^{-2|y|^{2} / t} d y \\
& \rightarrow C^{-1} \int\left|\left[e^{-\tilde{B}_{2}(y)}-e^{-\tilde{B}_{1}(y)}\right] \bar{u}\right|^{2} d y
\end{aligned}
$$

as $t \rightarrow \infty$. This last integral must be zero, by (6.6), so that $e^{-\tilde{B}_{2}(y)}=e^{-\tilde{B}_{1}(y)}$ for almost all $y$. (6.3) then shows that $e^{-\tilde{B}_{2}(\xi) t}=e^{-\tilde{B}_{1}(\xi) t}$ for all $t>0$ and almost all $\xi$. Differentiating with respect to $t$, we then conclude that $\tilde{B}_{2}=\tilde{B}_{1}$ a.e. 
Next we show that an effective artificial viscosity does indeed exist for the linearized Navier-Stokes system (1.4). Taking Fourier transforms in (1.4), we find that, in the notation of (6.1),

$$
i \xi_{j} A_{j}=\left[\begin{array}{cc}
0 & i \xi^{t} \\
i c^{2} \xi & 0
\end{array}\right]
$$

and

$$
B(\xi) \equiv \xi_{j} \xi_{k} B_{j k}=\left[\begin{array}{cc}
0 & 0 \\
0 & \varepsilon|\xi|^{2} I+\eta \xi \xi^{t}
\end{array}\right] .
$$

The symmetrizer $P$ is evidently given by

$$
P=\left[\begin{array}{ll}
c^{2} & 0 \\
0 & I
\end{array}\right],
$$

and the matrix $\xi_{j} A_{j}$ may be diagonalized: there is an invertible matrix $R(\xi)$ and a diagonal matrix $\Lambda(\xi)$ such that

$$
R^{-1}(\xi)\left(\xi_{j} A_{j}\right) R(\xi)=\Lambda(\xi)
$$

We define $\tilde{B}(\xi)$ to be the diagonal of $B(\xi)$ in the $R(\xi)$ coordinates:

$$
R^{-1}(\xi) \tilde{B}(\xi) R(\xi)=\operatorname{diag}\left[R^{-1}(\xi) B(\xi) R(\xi)\right]
$$

The symbols $\xi_{j} A_{j}$ and $\tilde{B}(\xi)$ therefore commute because $R^{-1}\left(\xi_{j} A_{j}\right) R$ and $R^{-1} \tilde{B} R$ are diagonal matrices, and (6.3) holds because $B$ is homogeneous of degree two, and $R$ of degree zero, in $\xi$.

To check the other two requirements, (6.5) and (6.6), we exploit the rotational symmetry to compute $\tilde{B}$ explicitly. Thus fix $\xi \in \mathbb{R}^{n}-\{0\}$ and choose an orthogonal matrix $V(\xi)$ such that $V^{t} \xi=|\xi| e_{1}$, where $e_{1}^{t}=(1,0, \ldots, 0)$. Setting

$$
Q(\xi)=\left[\begin{array}{cc}
1 & 0 \\
0 & V(\xi)
\end{array}\right]
$$

we then compute that

$$
Q^{t}\left(i \xi_{j} A_{j}\right) Q=\left[\begin{array}{cc}
W & 0 \\
0 & 0
\end{array}\right],
$$


where

$$
W=\left[\begin{array}{cc}
0 & i|\xi| \\
i c^{2}|\xi| & 0
\end{array}\right]
$$

Now,

$$
H^{-1} W H=\left[\begin{array}{cc}
-i c|\xi| & 0 \\
0 & i c|\xi|
\end{array}\right]
$$

where

$$
H=\left[\begin{array}{cc}
1 & 1 \\
-c & c
\end{array}\right]
$$

so that

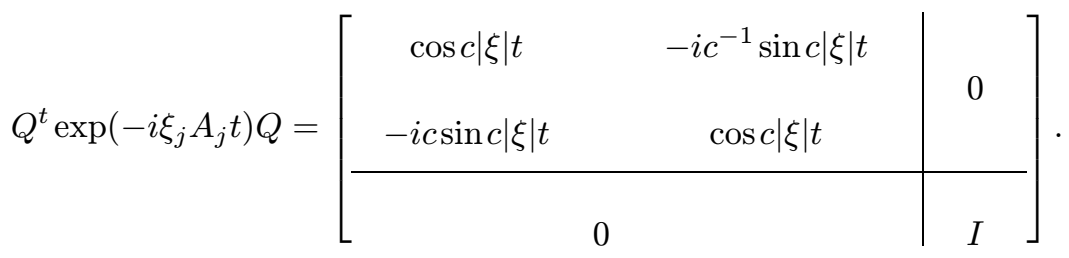

Evidently

$$
R(\xi)=Q(\xi)\left[\begin{array}{cc}
H & 0 \\
0 & I
\end{array}\right]
$$

whose norm is bounded above and below away from zero, uniformly in $\xi$ (this is also a consequence of the symmetrizability; see Theorem 2.1a, for example). Combining (6.8), (6.10), and (6.13), we then find that

$$
R^{-1} B R=\left[\begin{array}{cc|c}
\frac{1}{2} \nu|\xi|^{2} & -\frac{1}{2} \nu|\xi|^{2} & 0 \\
-\frac{1}{2} \nu|\xi|^{2} & \frac{1}{2} \nu|\xi|^{2} & \\
\hline & & \varepsilon|\xi|^{2} I
\end{array}\right],
$$

where $\nu=\varepsilon+\eta$. A straightforward computation based on (6.9) then shows finally that

$$
\tilde{B}(\xi)=\left[\begin{array}{cc}
\frac{1}{2}(\varepsilon+\eta)|\xi|^{2} & 0 \\
0 & \varepsilon|\xi|^{2} I+\frac{1}{2}(\eta-\varepsilon) \xi \xi^{t}
\end{array}\right] .
$$


The homogeneity (6.3) (which we have already checked) and the strict parabolicity (6.5) are now obvious. The approximation property (6.6) will be verified below. Observe first, however, that replacing $B(\xi)$ by $\tilde{B}(\xi)$ corresponds to replacing the linearized Navier-Stokes system (1.4) by the artificial viscosity system

$$
\left\{\begin{array}{l}
\rho_{t}+\operatorname{div} m=\frac{1}{2}(\varepsilon+\eta) \Delta \rho \\
m_{t}^{j}+c^{2} \rho_{x_{j}}=\varepsilon \Delta m^{j}+\frac{1}{2}(\eta-\varepsilon) \operatorname{div} m_{x_{j}} .
\end{array}\right.
$$

That is, (6.15) is the linear system whose Green's matrix $\tilde{G}$ is given by

$$
\hat{\tilde{G}}(\xi, t)=\exp \left[-\left(i \xi_{j} A_{j}+\tilde{B}(\xi)\right) t\right]
$$

Once (6.6) has been established for $\tilde{G}$, then, we will have proved that (6.15) is the canonical artificial viscosity system associated to the Navier-Stokes equations (1.1): (6.15) is strictly parabolic, the symbols of its first-order convection terms and second-order diffuson terms commute, and solutions of the original system (1.1) are asymptotic to those of (6.15) in the sense of (6.6). Actually, a much stronger asymptotic approximation result holds; we make this part of the following theorem, which summarizes these results:

Theorem 6.2. The unique effective artificial viscosity for the linearized Navier-Stokes system (1.4) is the mapping $\xi \mapsto \tilde{B}(\xi)$ given by (6.14). Moreover, if $G$ and $\tilde{G}$ are the respective Green's matrices for (1.4) and (6.15), given by (3.1) and (6.16), then for each nonnegative integer $k$ there are positive constants $C$ and $b$ such that, for $p \in[2, \infty], u_{0} \in L^{1} \cap W^{k, p}, t \geq 1$, and $|\alpha| \leq k$,

$$
\begin{aligned}
& \left\|D_{x}^{\alpha}[G(\cdot, t)-\tilde{G}(\cdot, t)] * u_{0}\right\|_{p} \\
\leq & t^{n / 2(1 / p-1)-|\alpha| / 2} C\left[t^{-1 / 2}\left\|u_{0}\right\|_{1}+e^{-b t}\left\|u_{0}\right\|_{W^{k, p}}\right] .
\end{aligned}
$$

Proof. We define a smooth cut-off function $\chi=\chi(|\xi|)$ such that $\chi=1$ for $|\xi| \leq R$ and $\chi=0$ for $|\xi| \geq R+1$, where $R$ is as in Theorem 3.2b. We then take $\widehat{G}_{1}=\chi \widehat{G}$ and $G_{2}=G-G_{1}$, as in Section 5 , so that by Lemma $5.2 \mathrm{~b}$,

$$
\begin{aligned}
\| D_{x}^{\alpha} & {[G(\cdot, t)-\tilde{G}(\cdot, t)] * u_{0} \|_{p} } \\
& \leq\left\|G_{2}(\cdot, t) * D_{x}^{\alpha} u_{0}\right\|_{p}+C\left\||\xi|^{|\alpha|}\left[\widehat{G}_{1}(\cdot, t)-\hat{\tilde{G}}(\cdot, t)\right] \hat{u}_{0}\right\|_{q} \\
& \leq C e^{-b t}\left\|u_{0}\right\|_{W^{k, p}}+C\left\|\hat{u}_{0}\right\|_{\infty}\left\||\xi|^{|\alpha|}[\chi \widehat{G}(\cdot, t)-\hat{\tilde{G}}(\cdot, t)]\right\|_{q},
\end{aligned}
$$


where $q$ is the Hölder conjugate of $p$. However, from Theorem 3.2a and (2.8),

$$
|\widehat{G}(\xi, t)| \leq C e^{-\vartheta|\xi|^{2} t},|\xi| \leq R
$$

and

$$
|\hat{\tilde{G}}(\xi, t)| \leq C e^{-\vartheta|\xi|^{2} t}, \xi \in \mathbb{R}^{n},
$$

for a positive constant $\vartheta$. Thus, for $r>0$,

$$
\left[\int_{|\xi| \geq r}|\xi|^{|\alpha| q}\left(|\chi \widehat{G}|^{q}+|\hat{\tilde{G}}|^{q}\right) d \xi\right]^{1 / q} \leq C(k) e^{-b t}
$$

and

$$
\begin{aligned}
\left\|D_{x}^{\alpha}[G(\cdot, t)-\tilde{G}(\cdot, t)] * u_{0}\right\|_{p} & \leq C e^{-b t}\left(\left\|u_{0}\right\|_{W^{k, p}}+\left\|u_{0}\right\|_{1}\right)+C\left\|u_{0}\right\|_{1} \\
& \times\left[\int_{|\xi| \leq r}|\xi|^{|\alpha| q}|\widehat{G}(\xi, t)-\hat{\tilde{G}}(\xi, t)|^{q} d \xi\right]^{1 / q} .
\end{aligned}
$$

It is therefore the low frequencies $|\xi| \leq r$ that are important here, and for these it is most convenient to work in the coordinates given by the matrix $Q(\xi)$ in (6.10). We therefore compute from (6.10), (6.12), and (6.14) that

$$
\begin{aligned}
Q^{t} \hat{\tilde{G}} Q & =\left(Q^{t} e^{-i \xi_{j} A_{j} t} Q\right)\left(Q^{t} e^{-\tilde{B}(\xi) t} Q\right) \\
& =\left[\begin{array}{cc}
\tilde{S} & 0 \\
0 & e^{-\varepsilon|\xi|^{2} t} I
\end{array}\right]
\end{aligned}
$$

where

$$
\tilde{S}=e^{-\nu|\xi|^{2} t / 2}\left[\begin{array}{cc}
\cos c|\xi| t & -i c^{-1} \sin c|\xi| t \\
-i c \sin c|\xi| t & \cos c|\xi| t
\end{array}\right],
$$

and again $\nu=\varepsilon+\eta$. On the other hand, if we write

$$
\widehat{G}=\left[\begin{array}{c|c}
g_{11} & -i g_{12} \xi^{t} \\
\hline-i c^{2} g_{12} \xi & e^{-\varepsilon|\xi|^{2} t} I+\left(g_{22}-e^{-\varepsilon|\xi|^{2} t}\right) \frac{\xi \xi^{t}}{|\xi|^{2}}
\end{array}\right],
$$

as in Lemma 3.1, we find that

$$
Q^{t} \widehat{G} Q=\left[\begin{array}{cc}
S & 0 \\
0 & e^{-\varepsilon|\xi|^{2} t} I
\end{array}\right],
$$


where

$$
S=\left[\begin{array}{cc}
g_{11} & -i g_{12}|\xi| \\
-i c^{2} g_{12}|\xi| & g_{22}
\end{array}\right] .
$$

We shall show that, for $r$ small, there are positive constants $C$ and $b$ such that

$$
|S(\xi, t)-\tilde{S}(\xi, t)| \leq C|\xi| e^{-\vartheta|\xi|^{2} t}, \quad 0 \leq|\xi| \leq r .
$$

Since $Q$ is orthogonal, it will then follow that

$$
\left[\int_{|\xi| \leq r}|\xi|^{|\alpha| q}|\widehat{G}(\xi, t)-\hat{\tilde{G}}(\xi, t)|^{q} d \xi\right]^{1 / q} \leq C t^{n / 2(1 / p-1)-|\alpha| / 2-1 / 2},
$$

which, together with (6.18), establishes the desired decay result (6.17).

(6.20) holds in a much more general setting, and can be derived by matrix perturbation techniques, as in Kawashima [8]. We shall check (6.20) directly, and for the upper left component only; the verification for the others is similar. First, we have from the definitions of $\lambda_{ \pm}(\xi)$ in Lemma 3.1 that, for small $|\xi|$,

$$
\lambda_{ \pm}(\xi)=-\frac{1}{2} \nu|\xi|^{2} \pm i c|\xi|(1+E)
$$

where $E$ is real and $|E| \leq C|\xi|^{2}$. The upper left entry of $S$ is then

$$
\begin{aligned}
g_{11} & =\frac{\lambda_{+} e^{\lambda-t}-\lambda_{-} e^{\lambda_{+} t}}{\lambda_{+}-\lambda_{-}} \\
& =e^{-\nu|\xi|^{2} t / 2} \cos [c|\xi| t(1+E)]+O\left(|\xi| e^{-\nu|\xi|^{2} t / 2}\right) \\
& =e^{-\nu|\xi|^{2} t / 2}\left[\cos c|\xi| t+O\left(|\xi|^{3} t\right)\right]+O\left(|\xi| e^{-\nu|\xi|^{2} t / 2}\right) \\
& =e^{-\nu|\xi|^{2} t / 2} \cos c|\xi| t+O\left(|\xi| e^{-\nu|\xi|^{2} t / 4}\right) .
\end{aligned}
$$

Comparison with (6.19) then shows that $S^{11}-\tilde{S}^{11}$ is bounded as required in (6.20).

Observe that the effective artificial viscosity system (6.15) satisfies the hypotheses of Theorem 2.3, so that, by the remarks made at the end of Section 2, the decay rate $\left\|D_{x}^{\alpha} \tilde{G}(\cdot, t) * u_{0}\right\|_{2} \sim t^{-(n / 4)-|\alpha| / 2}$ may indeed be attained. The same is therefore true also for solutions of the nonlinear Navier-Stokes system, for if $u(\cdot, t)$ is such a solution with initial data $u_{0}$, then

$$
u(\cdot, t)=\tilde{G}(\cdot, t) * u_{0}+[G(\cdot, t)-\tilde{G}(\cdot, t)] * u_{0}+\left[u(\cdot, t)-G(\cdot, t) * u_{0}\right] .
$$


Theorems 6.2 and 5.3 show that the $\alpha$-derivatives of the rightmost two terms here decay more rapidly than $t^{-n / 4-|\alpha| / 2}$. Consequently, if $u_{0}$ is chosen so that $\left\|D_{x}^{\alpha} \tilde{G}(\cdot, t) * u_{0}\right\|_{2} \geq C^{-1} t^{-n / 4-|\alpha| / 2}$, then the same lower bound will hold for the corresponding solution $u(\cdot, t)$.

We now assemble the conclusions of Theorems 2.3, 5.3, and 6.2 to give an asymptotic approximation result for solutions $u(\cdot, t)$ of the nonlinear NavierStokes system (4.2): for large time, $u(\cdot, t)$ is well-approximated by the convolution $\tilde{G}(\cdot, t) * u_{0}$, where $\tilde{G}$ is the Green's matrix for the linear artificial viscosity system $(6.15)$, as well as by the solution $\tilde{u}(\cdot, t)$ of the nonlinear artifical viscosity system (1.3), which consists of the nonlinear Euler equations together with the artificial viscosity of (6.15).

Theorem 6.3. Let $s=[n / 2]+1, \ell$ positive integer, and

$$
E=\max \left\{\left\|u_{0}\right\|_{H^{s+\ell}},\left\|u_{0}\right\|_{L^{1}}\right\} .
$$

Let $u$ be the solution of the nonlinear Navier-Stokes system (1.1), $\tilde{G}$ the Green's matrix (6.16) for the linear artificial viscosity system (6.15), and $\tilde{u}$ the solution of the nonlinear artificial viscosity system (1.3). Then if $E$ is sufficiently small,

$$
\begin{aligned}
\left\|D_{x}^{\alpha}\left[u(\cdot, t)-\tilde{G}(\cdot, t) * u_{0}\right]\right\|_{p},\left\|D_{x}^{\alpha}[u(\cdot, t)-\tilde{u}(\cdot, t)]\right\|_{p} \\
\leq C(|\alpha|) E L(t)(1+t)^{n / 2(1 / p-1)-|\alpha| / 2-1 / 2} \\
+E^{2} L(t)\left\{\begin{array}{c}
C(|\alpha|)(1+t)^{n / 2(1 / p-1)-1 / 2 \min \{|\alpha|,(\ell-3-|\alpha|)\}-1 / 2} \\
\text { for }|\alpha| \leq \ell-2,2 \leq p \leq \infty, \ell \geq 3 \\
C(|\alpha|, p)(1+t)^{n / 2(1 / p-1)-1 / 2 \min \{|\alpha|,(\ell-1-|\alpha|)\}-1 / 2} \\
\text { for }|\alpha| \leq \ell-1,2 \leq p<\infty .
\end{array}\right.
\end{aligned}
$$

Here $L(t)=\log (1+t)$ when $n=2$, and $L(t)=1$ for $n \geq 3$.

Proof. The estimate (6.23) for $u-\tilde{G} * u_{0}$ follows directly from the bound (5.29) for $u-G * u_{0}$ and the bound (6.17) for $G * u_{0}-\tilde{G} * u_{0}$. To prove the estimates for $u-\tilde{u}$, we recall the result (2.28) of Theorem 2.3, which applies to the system (1.3) to give a similar decay estimate for $\tilde{G} * u_{0}-\tilde{u}$. Combining this estimate with the bound above for $u-\tilde{G} * u_{0}$, we then obtain the required bound (6.23) for $u-\tilde{u}$.

We are now in a position to refine our description of the asymptotic behavior of the solution $\left[\begin{array}{c}\rho \\ m\end{array}\right]$ of the nonlinear Navier-Stokes system (1.1) in $L^{p}$ for $p \in$ 
$[2, \infty]$. The final results, stated in Theorems 6.7 and 6.8 below, are obtained from a detailed analysis of the Green's matrix $\tilde{G}$, together with the decay estimate (6.23) for the difference $u-\tilde{G} * u_{0}$.

To analyze $\tilde{G}$, we first observe that, by virtue of the commutativity (6.4), we may write

$$
\hat{\tilde{G}}(\xi, t)=\exp \left(-i \xi_{j} A_{j} t\right) \exp (-\tilde{B}(\xi) t)
$$

where $i \xi_{j} A_{j}$ and $\tilde{B}$ are given in (6.7) and (6.14). As in Section 2, we define $\hat{W}=(2 \pi)^{-n / 2} \exp \left(-i \xi_{j} A_{j} t\right)$ and $\hat{G}^{B}=\exp (-\tilde{B}(\xi) t) . W$ and $G^{B}$ are then the respective Green's matrices for the hyperbolic system (1.11) and the strictly parabolic system (1.10). Elementary computations based on (6.7) and (6.14) then enable us to identify $\tilde{G}=G^{B} * W$ explicitly:

Proposition 6.4. The Green's matrix $\tilde{G}$ for the artificial viscosity system (6.15) is given by

$$
\tilde{G}=\left[\begin{array}{cc}
w_{t} * K_{(\varepsilon+\eta) / 2} & -K_{\varepsilon} * \nabla w^{t}+\left(K_{\varepsilon}-K_{(\varepsilon+\eta) / 2}\right) * R * \nabla w^{t} \\
-c^{2} K_{(\varepsilon+\eta) / 2} * \nabla w & K_{\varepsilon} *(\delta I-R)+K_{(\varepsilon+\eta) / 2} * w_{t} * R
\end{array}\right],
$$

where, for $\vartheta>0, K_{\vartheta}$ is the heat kernel

$$
K_{\vartheta}(x, t)=(4 \pi \vartheta t)^{-n / 2} \exp \left(-\frac{|x|^{2}}{4 \vartheta t}\right)
$$

$w:[0, \infty) \rightarrow \mathcal{S}^{\prime}\left(\mathbb{R}^{n}\right)$ is the fundamental solution for the wave operator:

$$
\left\{\begin{array}{l}
w_{t t}-c^{2} \Delta w=0 \\
w(0)=0 \\
w_{t}(0)=\delta
\end{array}\right.
$$

and $R$ is the matrix of tempered distributions defined by $\widehat{R^{j k}}(\xi)=(2 \pi)^{-n / 2}$. $\left(\xi_{j} \xi_{k} /|\xi|^{2}\right)$.

Thus each entry of $\tilde{G}$ involves the convolution of a heat kernel with either $w_{t}$ or $\nabla w$, with the exception of the term $K_{\varepsilon} *(\delta I-R)$ in the $2-2$ entry. This term deserves special attention because, as we shall see, it is the term which dominates for large time. The following result, which is well known, explains the physical meaning of the convolution operator $(\delta I-R) *$ : 
Proposition 6.5. Let $m_{0} \in\left[\left(L^{2} \cap L^{1}\right)\left(\mathbb{R}^{n}\right)\right]^{n}$ be a given vector field. Then there exists a unique vector field $m_{0 f} \in\left(L^{2}\right)^{n}$ and a unique scalar function $g \in L^{2}+L^{\infty}$, such that $m_{0}=m_{0 f}+\nabla g$ and $\operatorname{div} m_{0 f}=0$. In fact, $m_{0 f}=$ $(\delta I-R) * m_{0}$, where $R$ is as in Proposition 6.4.

Thus $(\delta I-R) *$ is the operator which projects a given vector field onto its divergence-free part. Letting $\tilde{G}^{22}$ be the lower-right entry of the matrix $\tilde{G}$ in (6.24), and shifting by the constant field $m^{*}$, we may therefore write

$$
\tilde{G}^{22} *\left(m_{0}-m^{*}\right)=K_{\varepsilon} *\left(m_{0 f}-m^{*}\right)+K_{(\varepsilon+\eta) / 2} * w_{t} * \nabla g .
$$

The first term here can decay no more rapidly than at the rate of the heat kernel $K_{\varepsilon}$, but the second term will decay faster, owing to the appearance of the factor $w_{t}$, which, as we shall see, enhances the overall decay rate in $L^{p}$ for $p>2$. These observations therefore suggest that we decompose $\tilde{G}$ as follows:

$$
\tilde{G}=\tilde{G}_{f}+\tilde{G}_{\perp}, \text { where } \tilde{G}_{f}=\left[\begin{array}{cc}
0 & 0 \\
0 & K_{\varepsilon} *(\delta I-R)
\end{array}\right] .
$$

Now, in [6] we derive explicit, pointwise bounds in terms of $x$ and $t$ for the various entries of $\tilde{G}_{f}$ and $\tilde{G}_{\perp}$. These bounds are proved in a sequence of rather involved estimates for the convolution integrals appearing in the explicit representation (6.24) for $\tilde{G}$. We shall describe these bounds for a representative case at the end of this section, after establishing in Theorems 6.7 and 6.8 their relevance for solutions of the nonlinear Navier-Stokes system. For our present purposes, it suffices to simply quote the resulting $L^{p}$ decay rates for $\tilde{G}_{f}$ and $\tilde{G}_{\perp}$ :

Proposition 6.6. Let $\tilde{G}$ be the Green's matrix (6.24) for the artificial viscosity system (6.15), and let $\tilde{G}_{f}$ and $\tilde{G}_{\perp}$ be as above in (6.25). Then:

$$
\begin{aligned}
& \left\|D_{x}^{\alpha} \tilde{G}_{f}(\cdot, t)\right\|_{L^{p}} \\
\leq & C(|\alpha|) \begin{cases}\log t, & p=1 \text { and } \alpha=0, \\
t^{n / 2(1 / p-1)-|\alpha| / 2}, & |\alpha| \geq 0, p \in[1, \infty],(p, \alpha) \neq(1,0) ;\end{cases} \\
& \left\|D_{x}^{\alpha} \tilde{G}_{\perp}(\cdot, t)\right\|_{L^{p}} \\
\leq & C(|\alpha|) L_{\alpha}(t) t^{n / 2(1 / p-1)-|\alpha| / 2} \cdot t^{(n-1) / 4(2 / p-1)},|\alpha| \geq 0, p \in[1, \infty],
\end{aligned}
$$


where $L_{\alpha}(t)=\log (1+t)$ when $n=2$ and $\alpha=0$, and is otherwise identically one.

Thus $D_{x}^{\alpha} \tilde{G}_{f}$ decays at the rate $t^{n / 2(1 / p-1)-|\alpha| / 2}$, which is precisely that of a heat kernel. On the other hand, $D_{x}^{\alpha} \tilde{G}_{\perp}$ decays at the heat kernel rate times the factor $t^{(n-1) / 4(2 / p-1)}$. This latter factor is neutral when $p=2$, increases the overall decay rate for $p>2$, but retards the decay for $p<2$ (in fact, the bound in (6.27) may actually grow, rather than decay, when $p$ is close to 1 and $|\alpha|$ is small).

We now combine the estimates of Proposition 6.6, together with the bound for $u-\tilde{G} * u_{0}$ in Theorem 6.3 , to give a complete asymptotic description of solutions of the nonlinear Navier-Stokes system (1.1). As in Section 1, we let $\left[\begin{array}{c}\rho \\ m\end{array}\right]$ be the solution of (1.1) with initial data $\left[\begin{array}{c}\rho_{0} \\ m_{0}\end{array}\right]$ close to the constant state $\left[\begin{array}{c}\rho^{*} \\ m^{*}\end{array}\right]$, and we take $u=\left[\begin{array}{c}\rho-\rho^{*} \\ m-m^{*}\end{array}\right]$ and $u_{0}=\left[\begin{array}{c}\rho_{0}-\rho^{*} \\ m_{0}-m^{*}\end{array}\right]$. We decompose $u$ as follows:

$$
\begin{aligned}
u & =\left[\begin{array}{c}
\rho-\rho^{*} \\
m-m^{*}
\end{array}\right]=\tilde{G}_{f} * u_{0}+\tilde{G}_{\perp} * u_{0}+\left(u-\tilde{G} * u_{0}\right) \\
& =\left[\begin{array}{c}
0 \\
K_{\varepsilon} *(\delta I-R) *\left(m_{0}-m^{*}\right)
\end{array}\right]+\tilde{G}_{\perp} * u_{0}+\left(u-\tilde{G} * u_{0}\right) .
\end{aligned}
$$

We then have the following result, which gives our most complete description of the asymptotic behavior of $u$ in $L^{p}$ for $p \in[2, \infty]$ :

Theorem 6.7. Let $s=[n / 2]+1$ and $\ell$ a positive integer. Assume that initial data $\left[\begin{array}{c}\rho_{0} \\ m_{0}\end{array}\right]=u_{0}+\left[\begin{array}{c}\rho^{*} \\ m^{*}\end{array}\right]$ is given for which

$$
E=\max \left\{\left\|u_{0}\right\|_{H^{s+\ell}},\left\|u_{0}\right\|_{L^{1}}\right\}
$$

is sufficiently small, and let $\left[\begin{array}{c}\rho \\ m\end{array}\right]=u+\left[\begin{array}{c}\rho^{*} \\ m^{*}\end{array}\right]$ be the corresponding solution of the nonlinear Navier-Stokes system (1.1). Then the following decay rates apply to the three summands in the decomposition (6.28) of u:

$$
\begin{aligned}
& \left\|D_{x}^{\alpha}\left(\tilde{G}_{f} * u_{0}\right)(\cdot, t)\right\|_{L^{p}} \\
& \leq C(|\alpha|)\left\|u_{0}\right\|_{L^{1}} \begin{cases}\log t, & p=1 \text { and } \alpha=0, \\
t^{n / 2(1 / p-1)-|\alpha| / 2}, & |\alpha| \geq 0, p \in[1, \infty],(p, \alpha) \neq(1,0) ;\end{cases}
\end{aligned}
$$




$$
\begin{aligned}
& \left\|D_{x}^{\alpha}\left(\tilde{G}_{\perp} * u_{0}\right)(\cdot, t)\right\|_{L^{p}} \\
\leq & C(|\alpha|) L_{\alpha}(t)\left\|u_{0}\right\|_{L^{1}} t^{n / 2(1 / p-1)-|\alpha| / 2} t^{(n-1) / 4(2 / p-1)},|\alpha| \geq 0, p \in[1, \infty],
\end{aligned}
$$

where $L_{\alpha}=\log (1+t)$ when $n=2$ and $\alpha=0$, and is otherwise identically 1 ; and

$$
\begin{aligned}
& \left\|D_{x}^{\alpha}\left(u-\tilde{G} * u_{0}\right)(\cdot, t)\right\|_{L^{p}} \\
& \leq E^{2} L(t)\left\{\begin{array}{c}
C(|\alpha|)(1+t)^{n / 2(1 / p-1)-1 / 2 \min \{|\alpha|,(\ell-3-|\alpha|)\}-1 / 2} \\
\text { for }|\alpha| \leq \ell-2,2 \leq p \leq \infty, \ell \geq 3 \\
C(|\alpha|, p)(1+t)^{n / 2(1 / p-1)-1 / 2 \min \{|\alpha|,(\ell-1-|\alpha|)\}-1 / 2} \\
\text { for }|\alpha| \leq \ell-1,2 \leq p<\infty
\end{array}\right.
\end{aligned}
$$

where $L(t)=\log (1+t)$ when $n=2$, and is identically 1 when $n \geq 3$.

Thus, given sufficient initial regularity, the overall decay rate for $D_{x}^{\alpha} u$ is $t^{n / 2(1 / p-1)-|\alpha| / 2}$ in $L^{p}$ for $p \in[2, \infty]$, which is exactly that of a heat kernel. However, for $p>2,(6.30)$ and (6.31) show that the difference

$$
u-\tilde{G}_{f} * u_{0}=\left[\begin{array}{c}
\rho-\rho^{*} \\
m-m^{*}
\end{array}\right]-\left[\begin{array}{c}
0 \\
K_{\varepsilon} *(\delta I-R) *\left(m_{0}-m^{*}\right)
\end{array}\right]=\left[\begin{array}{c}
\rho-\rho^{*} \\
m-K_{\varepsilon} * m_{0 f}
\end{array}\right]
$$

decays more rapidly, by a factor of $t^{-q}$, where

$$
q=\min \left\{\frac{1}{2},(n-1)(1-2 / p) / 4\right\}
$$

It is therefore the first term on the right side of (6.28) which dominates for large time (in $\left.L^{p}, p \in(2, \infty]\right)$. Stated differently, the solution $\left[\begin{array}{c}\rho(\cdot, t) \\ m(\cdot, t)\end{array}\right]$ is asymptotic to the flow $\left[\begin{array}{c}\rho^{*} \\ K_{\varepsilon} * m_{0 f}\end{array}\right]$, which is incompressible with divergence-free velocity field. We have therefore arrived at the result that all smooth, small amplitude solutions of the nonlinear Navier-Stokes system (1.1) are asymptotically incompressible.

There is an additional point to be made concerning the decomposition (6.25) of $\tilde{G}$ and the asymptotic description of $u$ above. First, we may decompose the Green's matrix $W$ for the hyperbolic system (1.11) in the same way as in (6.25),

$$
W=W_{f}+W_{\perp}, \text { where } W_{f}=W *\left[\begin{array}{cc}
0 & 0 \\
0 & \delta I-R
\end{array}\right]
$$


so that $\tilde{G}_{f}=G^{B} * W_{f}$ and $\tilde{G}_{\perp}=G^{B} * W_{\perp}$. Now observe that the linearized Euler equations (1.11) have nontrivial steady-state solutions, namely, those flows $\left[\begin{array}{c}\rho-\rho^{*} \\ m-m^{*}\end{array}\right]$ for which $\rho=\rho^{*}$ and $\operatorname{div} m=0$. These steady-state solutions are thus nontrivial fixed points of the operator $W_{f}$, so that, in general, $W_{f} *\left[\begin{array}{c}\rho_{0}-\rho * \\ m_{0}-m *\end{array}\right]$ cannot decay. We therefore expect that the first term in the expansion (6.28)

$$
\tilde{G}_{f} *\left[\begin{array}{c}
\rho_{0}-\rho^{*} \\
m_{0}-m^{*}
\end{array}\right]=G^{B} *\left(W_{f} *\left[\begin{array}{c}
\rho_{0}-\rho^{*} \\
m_{0}-m^{*}
\end{array}\right]\right)
$$

will decay at most at the rate of a heat kernel; and this is precisely the result given in (6.29). The second term in the expansion (6.28) may be written

$$
\begin{aligned}
\tilde{G}_{\perp} *\left[\begin{array}{c}
\rho_{0}-\rho^{*} \\
m_{0}-m^{*}
\end{array}\right] & =G^{B} * W_{\perp} *\left[\begin{array}{c}
\rho_{0}-\rho^{*} \\
m_{0}-m^{*}
\end{array}\right] \\
& =G^{B} * W *\left[\begin{array}{c}
\rho_{0}-\rho^{*} \\
m_{0 f}-m^{*}
\end{array}\right] .
\end{aligned}
$$

One readily checks that, because $m_{0}-m_{0 f}$ is the gradient of a scalar function, both components of $W *\left[\begin{array}{c}\rho_{0}-\rho^{*} \\ m_{0 f}-m^{*}\end{array}\right]$ satisfy the standard wave equation. Since solutions of the wave equation decay in $L^{p}$ for $p>2$ (see Strauss [19]), we can expect that the entire term $G^{B} * W_{\perp} *\left[\begin{array}{c}\rho_{0}-\rho^{*} \\ m_{0}-m^{*}\end{array}\right]$ will decay at a rate which is faster than that determined separately by either $G^{B}$ or by the wave operator; and this is precisely the result (6.30).

Observe that the situation reverses itself entirely when $p<2$; the upper bound in (6.27) for $\tilde{G}_{\perp}$ may actually grow, rather than decay. We shall discuss $L^{p}$ rates of decay for $p<2$ at length in Sections 7 and 8.

The asymptotic description of the solution can be further refined when the initial data $u_{0}$ has a first moment: in this case the corresponding solution $u$ is asymptotically the Green's matrix $\tilde{G}$ itself, multiplied by the total mass vector of $u_{0}$ :

Theorem 6.8. Let $s, \ell$, and $E$ be as in Theorem 6.7, with $E$ sufficiently small, and assume that the initial data $u_{0}$ satisfes $(1+|x|) u_{0}(x) \in L^{1}$. Define

$$
U=\int_{\mathbb{R}^{n}} u_{0}(x) d x,
$$


and let $u$ be the solution of the nonlinear Navier-Stokes system (1.1) with initial data $u_{0}$. Then:

$$
\begin{aligned}
& \left\|D_{x}^{\alpha}[u(\cdot, t)-\tilde{G}(\cdot, t) U]\right\|_{p} \\
& \leq\left(E+\left\||x| u_{0}(x)\right\|_{L^{1}}\right)\left\{\begin{array}{c}
C(|\alpha|)(1+t)^{n / 2(1 / p-1)-1 / 2 \min \{|\alpha|,(\ell-3-|\alpha|)\}-1 / 2} \\
\text { for }|\alpha| \leq \ell-2,2 \leq p \leq \infty, \ell \geq 3 \\
C(|\alpha|, p)(1+t)^{n / 2(1 / p-1)-1 / 2 \min \{|\alpha|,(\ell-1-|\alpha|)\}-1 / 2} \\
\text { for }|\alpha| \leq \ell-1,2 \leq p<\infty .
\end{array}\right.
\end{aligned}
$$

Proof. In light of (6.23), we have only to prove (6.32) with $u$ replaced by $\tilde{G} * u_{0}$. Letting superscripts denote components, we have that

$$
D_{x}^{\alpha}\left[\left(\tilde{G}(\cdot, t) * u_{0}\right)^{j}(x)-(\tilde{G}(x, t) U)^{j}\right]=-\int_{0}^{1} \int D_{x}^{\alpha} \tilde{G}_{x_{\ell}}^{j k}(x-s y, t) y_{\ell} u_{0}^{k}(y) d y d s
$$

so that

$$
\begin{aligned}
& \left|D_{x}^{\alpha}\left(\tilde{G} * u_{0}-\tilde{G} U\right)(x, t)\right|^{p} \\
\leq & \left(\int_{0}^{1} \int\left|\nabla D_{x}^{\alpha} \tilde{G}(x-s y, t)\right|^{p}|y|\left|u_{0}(y)\right| d y d s\right)\left(\int|y|\left|u_{0}(y)\right| d y\right)^{p / q},
\end{aligned}
$$

where $q$ is the Hölder conjugate of $p$. Then

$$
\begin{aligned}
\left\|D_{x}^{\alpha}\left[\tilde{G} * u_{0}-\tilde{G} U\right]\right\|_{p}^{p} & \leq C\left\|\nabla D_{x}^{\alpha} \tilde{G}(\cdot, t)\right\|_{p}^{p}\left(\int|y|\left|u_{0}(y)\right| d y\right)^{1+p / q} \\
& \leq C\left(t^{n / 2(1 / p-1)-|\alpha| / 2-1 / 2}\left\||x| u_{0}(x)\right\|_{1}\right)^{p}
\end{aligned}
$$

by (2.6) of Theorem 2.1.

The result above gives the precise sense in which the asymptotic solution is a diffusion wave, and in fact defines the correct notion of diffusion wave in the present context. Recall the decomposition $\tilde{G}=W * G^{B}$, where $G^{B}$ and $W$ are the Green's matrices respectively for the strictly parabolic system (1.10) and the hyperbolic system (1.11). The asymptotic state in (6.32) may therefore be written

$$
\tilde{G}(\cdot, t) U=W(t) *\left[\tilde{G}^{B}(\cdot, t) U\right]=W(t) * \Theta(\cdot, t),
$$

where $\Theta(\cdot, t)$ is the solution of the parabolic system (1.10) with initial data $\delta U$. In light of the estimates of Theorem 2.1 for the Green's matrix $\tilde{G}^{B}(\cdot, t)$, we may 
regard $\Theta$ as a diffusion about the origin, so that $W * \Theta$ is a diffusion convected by the fundamental solution of the linearized Euler equations; that is, $W * \Theta$ is a "diffusion wave." (6.32) and (6.33) therefore show that, asymptotically, the solution $u(\cdot, t)$ of the nonlinear Navier-Stokes system is a diffusion wave whose total mass $U$ is the total mass of the initial data.

We conclude this section with a brief description and discussion of our pointwise bounds for $\tilde{G}$ in the representative case $n=3$. The following is a special case of the results of $[6]$ :

Proposition 6.9. Let $\tilde{G}$ be the Green's matrix of Proposition 6.6, and let $n=3$. Then for all $x$ and $t$,

$$
\left|\tilde{G}_{f}(x, t)\right| \leq C \min \left\{t^{-3 / 2},|x|^{-3}\right\}
$$

and

$$
\left|\tilde{G}_{\perp}(x, t)\right| \leq C t^{-2} \exp \left(-\frac{(|x|-c t)^{2}}{C t}\right) .
$$

The bound (6.34) for $\tilde{G}_{f}=G^{B} * W_{f}$ results from a straightforward estimate for the convolution $K_{\varepsilon} *(\delta I-R)$ appearing in (6.25). There is no hyperbolic effect in this term, since, as we saw above, the operator $W_{f} *$ has nontrivial fixed points. The bound for $\tilde{G}_{\perp}=G^{B} * W_{\perp}$ is considerably more interesting: the dissipative hyperbolic operator $W_{\perp}$ interacts with the three-dimensional diffusion $G^{B} \sim t^{-3 / 2} e^{-|x|^{2} / C t}$ by convecting the bulk of its mass along the light cone $\{x:|x|=c t\}$, and by increasing its pointwise decay rate by a factor of $t^{-1 / 2}$. The $L^{p}$ decay rates for $\tilde{G}_{f}$ and $\tilde{G}_{\perp}$ given in Proposition 6.6 then result simply by integrating the pointwise bounds (6.34) and (6.35). Complete technical details are given in [6].

7. Pointwise estimates of Green's matrices. Before studying behavior in $L^{p}, p \leq 2$, we address the general problem of converting frequency domain estimates on Green's matrices into pointwise estimates in the spatial domain.

General, symmetrizable, hyperbolic-strictly parabolic systems. We first consider systems

$$
u_{t}+A_{j} u_{x_{j}}-B_{j k} u_{x_{j}, x_{k}}=0
$$

for which there exists a symmetric matrix $P>0$ such that $P A_{j}$ is symmetric, for all $j$, and the operator $P B_{j k} u_{x_{j} x_{k}}$ is strictly parabolic. By the coordinate 
change $v=P^{1 / 2} u$, we can assume without loss of generality that the $A_{j}$ are symmetric, and that $B_{j k} u_{x_{j}, x_{k}}$ is strictly parabolic, i.e.

$$
\sum_{j, k} B_{j k} \xi_{j} \xi_{k} \geq \vartheta|\xi|^{2}, \quad \vartheta>0
$$

The Green's matrix $G(x, t)$ of (7.1) has transform

$$
\widehat{G}(\xi, t)=e^{\left(-i \xi_{j} A_{j}-B_{j k} \xi_{j} \xi_{k}\right) t} .
$$

Clearly, $\widehat{G}$ is an entire function of $\xi \in \mathcal{C}^{n}$.

Lemma 7.1. Let $C$ and $D$ be real $m \times m$ matrices. Then,

$$
\left|e^{(i C+D) t}\right| \leq e^{\bar{\sigma}\left(i\left(\left(C-C^{*}\right) / 2\right)+\left(\left(D+D^{*}\right) / 2\right)\right) t},
$$

where $|\cdot|$ is the standard operator norm over $\ell^{2}\left(\mathbb{R}^{n}\right)$ and $\bar{\sigma}(M)$ denotes the largest eigenvalue of $M$.

Proof. The vector function $v(t) \stackrel{\text { def }}{=} e^{(i C+D) t} v_{0}$ satisfies the ODE

$$
\frac{d}{d t} v=(i C+D) v ; \quad v(0)=v_{0} .
$$

From the energy estimate

$$
\begin{aligned}
\frac{d}{d t}|v|^{2} & =\left\langle v, \frac{d}{d t} v\right\rangle+\left\langle\frac{d}{d t} v, v\right\rangle \\
& =\left\langle v,\left[i\left(C-C^{*}\right)+\left(D+D^{*}\right)\right] v\right\rangle \\
& \leq \bar{\sigma}\left(i\left(C-C^{*}\right)+\left(D+D^{*}\right)\right)|v|^{2},
\end{aligned}
$$

we obtain

$$
|v(t)|^{2} \leq e^{\bar{\sigma}\left(i\left(\left(C-C^{*}\right) / 2\right)+\left(\left(D+D^{*}\right) / 2\right)\right) t}\left|v_{0}\right|^{2},
$$

or

$$
\left|e^{(i C+D) t} v_{0}\right| \leq e^{\bar{\sigma}\left(i\left(\left(C-C^{*}\right) / 2\right)+\left(\left(D+D^{*}\right) / 2\right)\right) t}\left|v_{0}\right| .
$$

Since $v_{0}$ is arbitrary, the result follows. 
Lemma 7.2. For any $\xi, \eta \in \mathbb{R}^{n}$,

$$
|\widehat{G}(\xi+i \eta, t)| \leq e^{\left(-(\vartheta / 2)|\xi|^{2}+\Lambda(\eta /|\eta|)|\eta|+\mu|\eta|^{2}\right) t},
$$

where $\widehat{G}$ is as in (7.3), $\vartheta>0$ is as in (7.2),

$$
\Lambda\left(\frac{\eta}{|\eta|}\right)=\frac{|A(\eta)|}{|\eta|} \stackrel{\text { def }}{=} \frac{\left|\sum_{j} A_{j} \eta_{j}\right|}{|\eta|},
$$

and $\mu$ is a positive constant.

Proof. Writing

$$
-i(\xi+i \eta)_{j} A_{j}-(\xi+i \eta)_{j}(\xi+i \eta)_{k} B_{j k}=i C+D,
$$

we have

$$
C=\left(-\xi_{j} A_{j}-\left(\xi_{j} \eta_{k}+\eta_{j} \xi_{k}\right) B_{j k}\right)
$$

and

$$
D=\left(\eta_{j} A_{j}-\xi_{j} \xi_{k} B_{j k}+\eta_{j} \eta_{k} B_{j k}\right)
$$

By symmetry of $A_{j}$,

$$
\bar{\sigma}\left(\frac{C-C^{*}}{2}\right) \leq K|\eta||\xi| \leq \frac{K}{\varepsilon}|\eta|^{2}+K \varepsilon|\xi|^{2},
$$

$\varepsilon>0$ arbitrary. By $(7.2),(7.8)$,

$$
\bar{\sigma}\left(\frac{D+D^{*}}{2}\right) \leq \Lambda\left(\frac{\eta}{|\eta|}\right)|\eta|+K|\eta|^{2}-\vartheta|\xi|^{2} .
$$

Thus, choosing $\varepsilon$ sufficiently small, we have

$$
\bar{\sigma}\left(i\left(\frac{C-C^{*}}{2}\right)+\left(\frac{D+D^{*}}{2}\right)\right)<\left(-\frac{\vartheta}{2}|\xi|^{2}+\Lambda\left(\frac{\eta}{|\eta|}\right)|\eta|+\mu|\eta|^{2}\right)
$$

for $\mu=K(1+1 / \varepsilon)$. The result now follows from Lemma 7.1.

Estimate (7.7) gives us pointwise bounds on $G(x, t)$, via the following weak version of the Paley-Wiener Theorem. 
Proposition 7.3. Let $\hat{f}(\xi)$ be an entire function of $\xi$ satisfying

$$
|\hat{f}(\xi+i \eta)| \leq C_{1}(1+|\xi| k)^{-N} e^{\mu_{1}(\eta /|\eta|)|\eta|+\mu_{2}|\eta|^{2}}
$$

for all $\xi, \eta \in \mathbb{R}^{n}$, for some $N>n$, where $\mu_{1}$ is a function from $S^{n}$ to $\mathbb{R}$ and $\mu_{2}$ is a positive constant. Then:

$$
|f(x)| \leq\left(\frac{C}{k^{n}}\right) e^{-\left[t\left(|x|-\mu_{1}(x /|x|)\right)^{+}\right]^{2} /\left(4 \mu_{2}\right)},
$$

where $(r)^{+} \stackrel{\text { def }}{=}(r+|r|) / 2$, and $C$ is a constant independent of $k, \mu_{j}$.

Proof. By the Fourier inversion formula, we have

$$
f(x)=(2 \pi)^{-n / 2} \int e^{i x \cdot \xi} \hat{f}(\xi) d \xi
$$

Since $\hat{f}$ is entire and rapidly decaying in $\xi$, we can move the surface of integration to $\xi^{\prime}=\xi+i \eta$, where

$$
\eta=\alpha \frac{x}{\sqrt{\mu_{2}}} ; \quad \alpha=\frac{\left(1-\mu_{1} /|x|\right)^{+}}{2 \sqrt{\mu_{2}}}
$$

and $\mu_{1}=\mu_{1}(\eta /|\eta|)=\mu_{1}(x|x|)$. We obtain

$$
\begin{aligned}
|f(x)| & =\left|\int e^{i x \cdot(\xi+i \eta)} \hat{f}(\xi+i \eta) d \xi\right| \\
& \leq e^{\left(\mu_{1}|\eta|+\mu_{2}|\eta|^{2}-\eta \cdot x\right)} \int C_{1}(1+|\xi| k)^{-N} d \xi \\
& =e^{\left|\eta \sqrt{\mu_{2}}-\left(\left(x-\left(\mu_{1} \eta\right) /|\eta|\right) /\left(2 \sqrt{\mu_{2}}\right)\right)\right|^{2}} e^{-\left|\left(x-\mu_{1}(\eta /|\eta|)\right) /\left(2 \sqrt{\mu_{2}}\right)\right|^{2}} \frac{C}{k^{n}}
\end{aligned}
$$

For $\alpha=0$, we have $\eta=0$, and (7.16) follows from the second line of (7.19). For $\alpha>0$, we have $\eta /|\eta|=x /|x|$, and the third line of (7.19) becomes

$$
\frac{C}{k^{n}} e^{-\left(\left(|x|-\mu_{1}\right) /\left(2 \sqrt{\mu_{2}}\right)\right)^{2}},
$$

giving the result. 
Corollary 7.4. The Green's matrix $G(x, t)$ of a symmetrizable, hyperbolicstrictly parabolic system, (7.1), satisfies

$$
\left|D_{x}^{\alpha} G(x, t)\right| \leq C(|\alpha|) t^{-n / 2-|\alpha| / 2} e^{-(|x|-\Lambda(x /|x|) t)^{+2} /(4 \mu t)},
$$

for some $\mu>0$, where $\Lambda(x /|x|)$ is as in (7.8), i.e., the maximum characteristic speed in the direction $x /|x|$ of the associated hyperbolic system,

$$
u_{t}+A_{j} u_{x_{j}}=0 .
$$

Proof. By Lemma 7.2, the entire function $\widehat{D_{x}^{\alpha} G}=(i \xi)^{\alpha} \widehat{G}$ satisfies the decay estimate

$$
\begin{aligned}
\left|\widehat{D_{x}^{\alpha} G}(\xi+i \eta)\right| & \leq C_{1}|\xi+i \eta|^{|\alpha|}\left(1+|\xi| t^{1 / 2}\right)^{-N} e^{\left(\Lambda((\eta /|\eta|))|\eta|+\mu|\eta|^{2}\right) t} \\
& \leq C t^{-|\alpha| / 2}\left(1+|\xi| t^{1 / 2}\right)^{|\alpha|-N} e^{\left(\Lambda(\eta /|\eta|)|\eta|+C \mu|\eta|^{2}\right) t}
\end{aligned}
$$

Applying Lemma 7.3, with $k=t^{1 / 2}, \mu_{1}=\Lambda t$, and $\mu_{2}=C \mu t$, and $N>n+|\alpha|$, we obtain the result.

According to the Corollary, $G(x, t)$ falls off like a heat kernel outside the cone of propagation, $\{x:|x| \leq \Lambda(x /|x|) t\}$ of system (7.1). This is the most we can say without specific information about the propagation of (7.1), or its interaction with the parabolic operator.

However, in the case of an artificial viscosity system, as described in Section 2 , we have the decomposition, due to commutation of $-i \xi_{j} A_{j}$ and $\xi_{j} \xi_{k} B_{j k}$, given by

$$
G=W * G^{B},
$$

where $W$ is the Green's matrix for $(7.23)$ and $G^{B}$ is the Green's matrix for the associated parabolic system,

$$
u_{t}=B_{j k} u_{x_{j} x_{k}}
$$

In this case, we can apply Lemma 7.2 and Proposition 7.3 to $G^{B}$, to obtain

$$
\left|D_{x}^{\alpha} G^{B}(x, t)\right| \leq C(|\alpha|) t^{-n / 2-|\alpha| / 2} e^{-|x|^{2} /(4 \mu t)} .
$$

Together with (7.24), (7.26) gives a fairly sharp pointwise description of $G(x, t)$. However, note (cf. calculations, Section 5) that precise decay information depends strongly on the details of the hyperbolic solution operator $W$. 
Linearized Navier-Stokes. In the case of nonstrictly parabolic systems with real viscosity, such as the linearized Navier-Stokes equations, we don not have estimate (7.7), and the argument above breaks down. However, we can easily establish a weaker pointwise bound sufficient for our purposes, using the $\xi$-derivative bounds of Lemma 3.2 and standard, real variable estimates.

Proposition 7.5. Let $G(x, t)$ be the Green's matrix of the linearized NavierStokes equations (1.4), and $G_{1}(x, t)$ be its smooth part, as defined in (5.9). Then

$$
\left|D_{x}^{\alpha} G_{1}(x, t)\right| \leq C_{N} t^{-n / 2-|\alpha| / 2}\left(\frac{|x|}{t}\right)^{-N} \text { for } t \geq 1,
$$

for any $N, x$. Moreover, as a distribution on $\mathbb{R}^{n}-\{0\}, G(\cdot, t)$ is a function satisfying

$$
|G(x, t)| \leq C_{N} \begin{cases}t^{-n / 2}\left(\frac{|x|}{t}\right)^{-N} & \text { for } t \geq 1, \\ |x|^{-N} & \text { for } t \leq 1\end{cases}
$$

for any $N>n$.

Proof. By Hausdorff-Young,

$$
\begin{aligned}
\left|x^{\beta} G(x, t)\right| & \leq\left\|\widehat{x^{\beta} G}(\cdot, t)\right\|_{1} \\
& =\left\|D_{\xi}^{\beta} \widehat{G}(\cdot, t)\right\|_{1},
\end{aligned}
$$

while

$$
\begin{aligned}
\left|x^{\beta} D_{x}^{\alpha} G_{1}(x, t)\right| & \leq\left\|x^{\widehat{\beta} D_{x}^{\alpha} G_{1}}(\cdot, t)\right\|_{1} \\
& =\left\|D_{\xi}^{\beta}\left(\xi^{\alpha} \widehat{G}_{1}(\xi, t)\right)\right\|_{1} .
\end{aligned}
$$

Thus, it is sufficient to show that, for $|\beta|=N$,

$$
\left\|D_{\xi}^{\beta} \widehat{G}(\xi, t)\right\|_{1} \leq C_{N} \begin{cases}t^{N-(n / 2)} & t \geq 1 \\ 1 & t \leq 1,\end{cases}
$$

when $N>n$, and

$$
\left\|D_{\xi}^{\beta}\left(\xi^{\alpha} \widehat{G}_{1}(\xi, t)\right)\right\|_{1} \leq C_{N} t^{N-(n / 2)-|\alpha| / 2} \quad \text { for } t \geq 1 .
$$

But, (7.31) - (7.32) follow easily from the $\xi$-derivative bounds given in Lemma 3.2 .

At the expense of further effort, we can in fact recover a close approximation of (7.21), by interpolating between the real and complex analytic arguments. We present this result for completeness, though it is not needed for our later analysis. 
Lemma 7.6. Let $r^{2}=(\xi+i \eta) \cdot(\xi+i \eta)=\left(|\xi|^{2}-|\eta|^{2}\right)+i(\xi \cdot \eta)$. Then, there exists $R>0$ such that:

(i) For $\left|r^{2}\right|<R^{2}$,

$$
\left|D_{\xi+i \eta}^{\alpha} \widehat{G}(\xi+i \eta, t)\right| \leq C(1+|\xi|+|\eta|)^{|\alpha|}(1+t)^{|\alpha|} e^{\left(-\vartheta|\xi|^{2}+c|\eta|+\mu|\eta|^{2}\right) t},
$$

and

(ii) For $\left|r^{2}\right| \geq R^{2}$,

$$
\left|D_{\xi+i \eta}^{\alpha} \widehat{G}(\xi+i \eta, t)\right| \leq C\left(\frac{|\xi|+|\eta|}{|r|}\right)^{|\alpha|}\left(e^{-a t / 2}|r|^{-|\alpha|-1}+e^{-\vartheta r^{2} t}|r|^{-|\alpha|}\right)
$$

where $a, \vartheta>0$.

Proof. Note that $r^{2}=|\xi|^{2}$ in the case $|\eta|=0$, in agreement with the notation of Section 3. This has the consequence that expressions (3.1)-(3.2), and (3.28)-(3.29) for $\widehat{G}(\xi, t)$ remain valid for $\widehat{G}(\xi+i \eta, t)$ if we everywhere replace $\xi$ by $\xi+i \eta$ and $|\xi|^{2}$ by $r^{2}$. For, it is easily checked that these expressions are analytic functions of $r^{2}$ : (3.28)- (3.29) by construction, and (3.1) by the observation that terms such as $\left(e^{\lambda_{+} t}-e^{\lambda_{-} t}\right) /\left(\lambda_{+}-\lambda_{-}\right)$are invariant under permutation of $\lambda_{-}$and $\lambda_{+}$and bounded at the branch point $\lambda_{-}=\lambda_{+}$, hence analytic despite the branch singularity in $\lambda_{ \pm}\left(r^{2}\right)$. As entire expressions agreeing with $\widehat{G}$ on $|\eta|=0$, they must be valid also for arbitrary $\eta$. The same result can equally well be obtained by rewording the derivations of (3.1), (3.28), (3.29) in terms of $r^{2}$. With this observation, it is easy to extend the bounds of Theorem 3.2 to the complex plane. For example, the series expansion (3.12) at $|\xi|=\infty$ is valid, more generally, around $\left|r^{2}\right|=\infty$, by the same derivation; in one space dimension, this reduces to the fact that $\widehat{G}(\xi+i \eta, t)$ is holomorphic at $|\xi+i \eta|=\infty$ (cf. [16]). Likewise, (3.15) carries over in modified form, with the observation that, for $\left|r^{2}\right| \leq R^{2}$

$$
\operatorname{Re} \lambda_{\mp}\left(r^{2}\right) \leq-\vartheta|\xi|^{2}+\mu|\eta|^{2}+c|\eta|,
$$

which follows by direct calculation from (3.2). Carrying through the analysis of Theorem 3.2, line by line, with $r^{2}$ substituted for $|\xi|^{2}$, and noting that $\partial\left(r^{2}\right) / \partial(\xi+i \eta)=2(\xi+i \eta)$, we obtain the claimed bounds.

Proposition 7.7. For $|x|>0$,

$$
\left|D_{x}^{\beta} G(x, t)\right| \leq C\left(1+t^{-2 n-2|\beta|-1}\right) t^{-n / 2-|\beta| / 2}\left(\frac{|x|}{t}\right)^{-n-|\beta|} e^{-(|x|-c t)^{+2} / 4 \mu t} .
$$


Proof. First, consider the case $|\beta|=0$. Taking $|\alpha|=n$ and combining (7.33)- (7.34), we have

$$
\begin{aligned}
\left|\widehat{x^{\alpha} G}(\xi+i \eta, t)\right| & =\left|D_{\xi}^{\alpha} \widehat{G}\right| \\
& \leq C\left(\frac{|\xi|+|\eta|}{R+|r|}\right)^{2 n+1}(1+|\xi|)^{-1-n} e^{-a t / 2}+C t^{n} e^{\left(-\vartheta|\xi|^{2}+\mu|\eta|^{2}+c|\eta|\right) t} .
\end{aligned}
$$

With

$$
\begin{aligned}
\left|\frac{|\xi|+|\eta|}{R+|r|}\right| & \leq C\left(\frac{\left|r^{2}\right|+2|\eta|^{2}}{R^{2}+\left|r^{2}\right|}\right)^{1 / 2} \\
& \leq C(1+|\eta|) \\
& \leq C(1+t) e^{\mu|\eta|^{2} t}
\end{aligned}
$$

this gives

$$
\left|\widehat{x^{\alpha} G}(\xi+i \eta, t)\right| \leq C\left(1+t^{-2 n-1}\right)(1+t)^{n}(1+|\xi| t)^{-n-1} e^{\mu|\eta|^{2} t+c|\eta| t},
$$

for $|\alpha|=n$. Applying Proposition 7.3, we have the result. The case $|\beta|>0$ follows similarly, using $|\alpha|=n+|\beta|$.

Corollary 7.8. For $|x|>0$,

$$
\begin{aligned}
& \left|D_{x}^{\beta}(G-\tilde{G})(x, t)\right| \\
& \quad \leq C\left(1+t^{-2 n-2|\beta|-1}\right) t^{-n / 2-|\beta| / 2-1 / 2}\left(\frac{|x|}{t}\right)^{-n-|\beta|} e^{-(|x|-c t)^{+2} /(4 \mu t)} .
\end{aligned}
$$

Proof. This follows by the argument of Proposition 7.7, after the observation that, for $\left|r^{2}\right|<R^{2}$,

$$
\begin{aligned}
& \left|D_{\xi+i \eta}^{\alpha}(\hat{G}-\hat{\tilde{G}})(\xi+i \eta, t)\right| \\
& \quad \leq C|r|(1+|\xi|+|\eta|)^{|\alpha|}(1+t)^{|\alpha|} e^{\left(-\vartheta|\xi|^{2}+c|\eta|+\mu|\eta|^{2}\right) t}
\end{aligned}
$$

The bound (7.35) follows by expanding $\hat{\tilde{G}}$ as in (3.1), by the technique used to expand $\widehat{G}$, and comparing directly.

Remark. Proposition 7.7 and Corollary 7.8 imply that, for any $\sigma>0$,

$$
\left|D_{x}^{\beta} G(x, t)\right| \leq C(|\beta|) t^{-n / 2-|\beta| / 2} e^{-(|x|-\Lambda(x /|x|) t)^{+2} / 4 \mu t^{1+\sigma}},
$$

and

$$
\left|D_{x}^{\beta}(G-\tilde{G})(x, t)\right| \leq C(|\beta|) t^{-n / 2-|\beta| / 2-1 / 2} e^{-(|x|-\Lambda(x /|x|) t)^{+2} / 4 \mu t^{1+\sigma}},
$$


for $|x|>0$. In dimension $n=1, \sigma=0$ can be attained by taking into account cancellation in terms of order $|\xi|^{-1}$, rather than differentiating with respect to $\xi$.

8. Decay to diffusion waves in $L^{p}, p<2$. Using the pointwise bounds derived in Section 7, we can now establish the decay and asymptotic behavior of solutions of (1.1) in $L^{p}$ for $p<2$.

Decompose the Green's matrix $G(x, t)$ of the linearized Navier-Stokes equations, (1.4), as

$$
G=\tilde{G}+\left(G_{1}-\tilde{G}_{1}\right)+\left(G_{2}-\tilde{G}_{2}\right),
$$

where $\tilde{G}(x, t)$ is the Green's function of the associated, effective artificial viscosity system, (1.7), and $\widehat{G}_{j}, \hat{\tilde{G}}_{j}$ are as in (5.9), with $R$ sufficiently large. By Lemma 5.2 applied to $G$ and Proposition 4.3 applied to $\tilde{G}$, the term $\left(G_{2}-\tilde{G}_{2}\right)$ is negligible, satisfying

$$
\left(G_{2}-\tilde{G}_{2}\right)=e^{-b t} \widehat{M(\xi, t)}
$$

and

$$
D_{x_{j}}\left(G_{2}-\tilde{G}_{2}\right) \cdot\left[\begin{array}{ll}
0 & 0 \\
0 & I
\end{array}\right]=e^{-b t}\left(1+t^{-1 / 2}\right) \widehat{\mathcal{M}(\xi, t)},
$$

where $b>0$ and $M$ and $\mathcal{M}$ are strong and ordinary $L^{p}$ multipliers, respectively.

Lemma 8.1. For all $p \leq 2, \sigma>0$,

$$
\left\|D_{x}^{\alpha}\left(G_{1}-\tilde{G}_{1}\right)\right\|_{p} \leq C(|\alpha|, \sigma)(1+t)^{n / 2(1 / p-1)-|\alpha| / 2+(n / 4)(2 / p-1)-1 / 2+\sigma} .
$$

Proof. For $t \leq 1$, the result follows from (5.10) in Lemma 5.2. Thus, we may assume $t \geq 1$. From (6.21), we have

$$
\left\|D_{x}^{\alpha}\left(G_{1}-\tilde{G}_{1}\right)\right\|_{2} \leq C t^{-n / 4-|\alpha| / 2-1 / 2}
$$

while from (7.27) and (7.21) we have

$$
\left|D_{x}^{\alpha}\left(G_{1}-\tilde{G}_{1}\right)(x, t)\right| \leq C_{N} t^{-n / 2-|\alpha| / 2}\left(\frac{|x|}{t}\right)^{-N}, \quad \text { for } t \geq 1,
$$

for any $N>n$. 
Fixing $t$, let

$$
\Omega^{j} \stackrel{\text { def }}{=}\left\{x: j \leq \frac{|x|}{t} \leq j+1\right\},
$$

and denote $\|f\|_{L^{p}\left(\Omega^{j}\right)}$ by $\|f\|_{p, \Omega^{j}}$. By Hölder's inequality, we have

$$
\|f\|_{p, \Omega^{j}} \leq\|f\|_{p^{\prime}, \Omega^{j}}\left|\Omega^{j}\right|^{1 / p-1 / p^{\prime}}, \text { for } p \leq p^{\prime} .
$$

A second application of Hölder's inequality gives

$$
\begin{aligned}
\left\|D_{x}^{\alpha}\left(G_{1}-\tilde{G}_{1}\right)\right\|_{2+\sigma, \Omega^{j}} & \\
\leq & \left\|D_{x}^{\alpha}\left(G_{1}-\tilde{G}_{1}\right)\right\|_{2, \Omega^{j}}^{2 /(2+\sigma)}\left\|D_{x}^{\alpha}\left(G_{1}-\tilde{G}_{1}\right)\right\|_{\infty, \Omega^{j}}^{\sigma /(2+\sigma)} \\
& \leq\left(C t^{-n / 4-|\alpha| / 2-1 / 2}\right)^{2 /(2+\sigma)}\left(C_{N} t^{-n / 2-|\alpha| / 2} j^{-N}\right)^{\sigma /(2+\sigma)}
\end{aligned}
$$

for any $\sigma>0, j \geq 1$. Applying (8.8) and (8.6), we have, for $j \geq 1, p \leq 2$, and $\sigma$ small (depending on $n$ ),

$$
\begin{aligned}
& \left\|D_{x}^{\alpha}\left(G_{1}-\tilde{G}_{1}\right)\right\|_{p, \Omega^{j}} \\
\leq & \left\|D_{x}^{\alpha}\left(G_{1}-\tilde{G}_{1}\right)\right\|_{2+\sigma, \Omega^{j}}\left|\Omega^{j}\right|^{1 / p-1 /(2+\sigma)} \\
\leq & C\left(t^{-n / 4-|\alpha| / 2-1 / 2+\sigma_{2}}\right) j^{-(N \sigma /(2+\sigma))+1}\left((j t)^{n-1} t\right)^{1 / p-1 / 2} \\
\leq & C\left(j^{(n-1) / 2-(N \sigma /(2+\sigma))+1}\right)\left(t^{\sigma_{2}}\right)\left(t^{-n / 2(1-1 / p)-|\alpha| / 2+(n / 4)(2 / p-1)-1 / 2}\right),
\end{aligned}
$$

where $\sigma_{2}(\sigma)>0$ goes to zero as $\sigma \rightarrow 0$, while

$$
\begin{aligned}
\left\|D_{x}^{\alpha}\left(G_{1}-\tilde{G}_{1}\right)\right\|_{p, \Omega^{0}} & \leq\left\|D_{x}^{\alpha}\left(G_{1}-\tilde{G}_{1}\right)\right\|_{2}\left|\Omega^{0}\right|^{1 / p-1 / 2} \\
& \leq C t^{-n / 2(1-1 / p)-|\alpha| / 2+n / 4(2 / p-1)-1 / 2}
\end{aligned}
$$

Choosing $N$ so large that $(n-1) / 2-N \sigma /(2+\sigma)+1<-1$, and summing over $j$, we have the result.

Theorem 8.2. Let $u_{0} \in H^{s+\ell} \cap L^{1}\left(\mathbb{R}^{n}\right)$, for $s \geq[n / 2]+1$ and $\ell$ a positive integer. For

$$
E=\max \left\{\left\|u_{0}\right\|_{H^{s+\ell}},\left\|u_{0}\right\|_{W^{\ell-1,1}}\right\}
$$


sufficiently small, the solution u of (4.1) satisfies

$$
\leq E\left\{\begin{array}{r}
C(|\alpha|) L_{\alpha}(t)(1+t)^{n / 2(1 / p-1)-1 / 2 \min \{|\alpha|, \ell-2-|\alpha|\}+((n-1) / 4)(2 / p-1)} \\
\text { for }|\alpha| \leq \ell-2,1 \leq p \leq 2, \ell \geq 3, \\
C(|\alpha|, p) L_{\alpha}(t)(1+t)^{n / 2(1 / p-1)-1 / 2 \min \{|\alpha|, \ell-1-|\alpha|\}+((n-1) / 4)(2 / p-1)} \\
\text { for }|\alpha| \leq \ell-1,1<p \leq 2,
\end{array}\right.
$$

and, for any $\sigma>0$,

$$
\left\|D_{x}^{\alpha}(u-\tilde{u})\right\|_{p},\left\|D_{x}^{\alpha}\left(u-G * u_{0}\right)\right\|_{p},\left\|D_{x}^{\alpha}\left(u-\tilde{G} * u_{0}\right)\right\|_{p}
$$

$$
\leq E\left\{\begin{array}{r}
C(|\alpha|, \sigma)(1+t)^{n / 2(1 / p-1)-1 / 2 \min \{|\alpha|, \ell-3-|\alpha|\}+(n / 4)(2 / p-1)-1 / 2+\sigma} \\
\text { for }|\alpha| \leq \ell-2,1 \leq p \leq 2, \ell \geq 3, \\
C(|\alpha|, p, \sigma)(1+t)^{n / 2(1 / p-1)-1 / 2 \min \{|\alpha|,(\ell-1-|\alpha|)\}+(n / 4)(2 / p-1)-1 / 2+\sigma} \\
\text { for }|\alpha| \leq \ell-1,1<p \leq 2,
\end{array}\right.
$$

where $\tilde{u}$ is the solution of the associated nonlinear artificial viscosity system (1.5). Here, $L_{\alpha}(t)=\log (1+t)$ when $n=2$ and $\alpha=0$ and is otherwise one, and $L(t)=$ $\log (1+t)$ when $n=2$ and is otherwise one. If, further, $(1+|x|) u_{0}(x) \in L^{1}$, then

$$
\leq\left(E+\left\|x u_{0}\right\|_{1}\right)\left\{\begin{array}{r}
C(|\alpha|, \sigma)(1+t)^{n / 2(1 / p-1)-1 / 2 \min \{|\alpha|, \ell-3-|\alpha|\}+(n / 4)(2 / p-1)-1 / 2+\sigma} \\
\text { for }|\alpha| \leq \ell-2,1 \leq p \leq 2, \ell \geq 3, \\
C(|\alpha|, p, \sigma)(1+t)^{n / 2(1 / p-1)-1 / 2 \min \{|\alpha|, \ell-1-|\alpha|\}+(n / 4)(2 / p-1)-1 / 2+\sigma} \\
\text { for }|\alpha| \leq \ell-1,1<p \leq 2,
\end{array}\right.
$$

where $U=\int_{\mathbb{R}^{n}} u_{0} d x$.

Proof. Let $|\alpha| \leq \ell-2, \ell \geq 3$. By Proposition 6.6, we have

$$
\begin{aligned}
& \left\|D_{x}^{\alpha} \tilde{G} * u_{0}\right\|_{p} \\
\leq & \min \left\{\left\|D_{x}^{\alpha} \tilde{G}\right\|_{p}\left\|u_{0}\right\|_{1},\|\tilde{G}\|_{1}\left\|D_{x}^{\alpha} u_{0}\right\|_{p}\right\} \\
\leq & E C(|\alpha|) L_{\alpha}(t)(1+t)^{n / 2(1 / p-1)-|\alpha| / 2+((n-1) / 4)(2 / p-1)} .
\end{aligned}
$$


Likewise, (8.4) and (8.2) give, respectively,

$$
\begin{aligned}
& \left\|D_{x}^{\alpha}\left(G_{1}-\tilde{G}_{1}\right) * u_{0}\right\|_{p} \\
\leq & \left\|D_{x}^{\alpha}\left(G_{1}-\tilde{G}_{1}\right)\right\| p\left\|u_{0}\right\|_{1} \\
\leq & C(|\alpha|, \sigma) E(1+t)^{n / 2(1 / p-1)-|\alpha| / 2+(n / 4)(2 / p-1)-1 / 2+\sigma},
\end{aligned}
$$

and

$$
\begin{aligned}
\left\|D_{x}^{\alpha}\left(G_{2}-\tilde{G}_{2}\right) * u_{0}\right\|_{p} & \leq C e^{-b t}\left\|D_{x}^{\alpha} u_{0}\right\|_{p} \\
& \leq C E e^{-b t} .
\end{aligned}
$$

Following (5.7), write

$$
\begin{aligned}
D_{x}^{\alpha} u(t) & =G(t) * D_{x}^{\alpha} u_{0}+\int_{0}^{t} \sum_{k} D_{x_{k}} G(t-s) * D_{x}^{\alpha} Q^{k}(s) d s \\
& =I+I I .
\end{aligned}
$$

We can immediately bound the linear term $I$ by

$$
\begin{aligned}
\|I\|_{p} & \leq\left\|D_{x}^{\alpha} \tilde{G} * u_{0}\right\|_{p}+\left\|D_{x}^{\alpha}\left(G_{1}-\tilde{G}_{1}\right) * u_{0}\right\|_{p}+\left\|D_{x}^{\alpha}\left(G_{2}-\tilde{G}_{2}\right) * u_{0}\right\|_{p} \\
& \leq E C L_{\alpha}(t)(1+t)^{n / 2(1 / p-1)-|\alpha| / 2+((n-1) / 4)(2 / p-1)},
\end{aligned}
$$

using (8.15)-(8.17) and the fact that $\frac{1}{4}(2 / p-1)>-\frac{1}{2}$.

To bound the nonlinear term $I I$, we observe that the source term $Q$, being quadratic, can be estimated using the decay rates for $L^{p}, p \geq 2$, already known from Theorem 5.3. For example, as in (5.36),

$$
\begin{aligned}
\int_{0}^{t}\|Q\|_{1} d s & \leq C \int_{0}^{t}\left\||u|^{2}+|u|\left|D_{x} u\right|\right\|_{1} d s \\
& \leq C \int_{0}^{t}\left(\|u\|_{2}^{2}+\left\|D_{x} u\right\|_{2}^{2}\right) d s \\
& \leq C(|\alpha|) E^{2} L(t) .
\end{aligned}
$$

Likewise, for $Q_{1}$ and $Q_{2}$ as defined in (5.3) we have, similarly to (5.38) and (5.39),

$$
\begin{gathered}
\left.\left\|D_{x}^{\alpha} Q_{1}\right\|_{1}\right\} \\
\left.\qquad D_{x}^{\beta} Q_{2} \|_{1},|\beta|=|\alpha|-1\right\} \\
\leq \sup _{\sum\left|\gamma_{j}\right|=|\alpha|}\left\|D_{x}^{\gamma_{1}} u\right\|_{2}\left\|D_{x}^{\gamma_{2}} u\right\|_{2} \prod_{j \geq 3}\left\|D_{x}^{\gamma_{j}} u\right\|_{\infty} \\
\leq C(|\alpha|) E^{2}(1+t)^{-n / 2-1 / 2 \min \{|\alpha|, \ell-2-|\alpha|\}}
\end{gathered}
$$

and 


$$
\begin{array}{r}
\left\|D_{x} D_{x}^{\alpha} Q_{j}\right\|_{p} \leq C \sup _{\sum\left|\gamma_{j}\right|=|\alpha|+j}\left\|D_{x}^{\gamma_{1}} u\right\|_{2 p /(2-p)}\left\|D_{x}^{\gamma_{2}} u\right\|_{2} \prod_{j \geq 3}\left\|D_{x}^{\gamma_{j}} u\right\|_{\infty} \\
\leq C(|\alpha|) E^{2}(1+t)^{n / 2(1 / p-1)-1 / 2 \min \{|\alpha|, \ell-3-|\alpha|\}-1 / 2}, \\
|\alpha| \leq \ell-2 .
\end{array}
$$

Thus, similarly to (5.41), (5.42), we have, for $|\alpha|>0,|\beta|=|\alpha|-1$,

$$
\|I I\|_{p}
$$

$$
\begin{aligned}
& \leq \int_{0}^{t / 2}\left\|D_{x}^{\alpha} D_{x} \tilde{G} * Q\right\|_{p}+\int_{t / 2}^{t}\left\|D_{x} \tilde{G} * D_{x}^{\alpha} Q_{1}\right\|_{p}+\int_{t / 2}^{t}\left\|D_{x}^{2} \tilde{G} * D_{x}^{\beta} Q_{2}\right\|_{p} . \\
& \quad+\int_{0}^{t / 2}\left\|D_{x}^{\alpha} D_{x}\left(G_{1}-\tilde{G}_{1}\right) * Q\right\|_{p}+\int_{t / 2}^{t}\left\|D_{x}\left(G_{1}-\tilde{G}_{1}\right) * D_{x}^{\alpha} Q_{1}\right\|_{p} . \\
& \quad+\int_{t / 2}^{t}\left\|D_{x}^{2}\left(G_{1}-\tilde{G}_{1}\right) * D_{x}^{\beta} Q_{2}\right\|_{p}+\int_{0}^{t}\left\|\left(G_{2}-\tilde{G}_{2}\right) * D_{x} D_{x}^{\alpha} Q\right\|_{p} \\
& \leq \int_{0}^{t / 2}\left\|D_{x}^{\alpha} D_{x} \tilde{G}\right\|_{p}\|Q\|_{1}+\int_{t / 2}^{t}\left\|D_{x} \tilde{G}\right\|_{p}\left\|D_{x}^{\alpha} Q_{1}\right\|_{1}+\int_{t / 2}^{t}\left\|D_{x}^{2} \tilde{G}\right\|_{p}\left\|D_{x}^{\beta} Q_{2}\right\|_{1} \\
& \quad+\int_{0}^{t / 2}\left\|D_{x}^{\alpha} D_{x}\left(G_{1}-\tilde{G}_{1}\right)\right\|\left\|_{p}\right\| Q\left\|_{1}+\int_{t / 2}^{t}\right\| D_{x}\left(G_{1}-\tilde{G}_{1}\right)\left\|_{p}\right\| D_{x}^{\alpha} Q_{1} \|_{1} \\
& \quad+\int_{t / 2}^{t}\left\|D_{x}^{2}\left(G_{1}-\tilde{G}_{1}\right)\right\|_{p}\left\|D_{x}^{\beta} Q_{2}\right\|_{1}+\int_{0}^{t} e^{-b(t-s)}\left\|D_{x} D_{x}^{\alpha} Q\right\|_{p} \\
& \leq C(|\alpha|)(1+t)^{n / 2(1 / p-1)-|\alpha| / 2+((n-1) / 4)(2 / p-1)-1 / 2} \int_{0}^{t / 2}\|Q(s)\|_{1} d s
\end{aligned}
$$$$
+C(|\alpha|) E^{2}(1+t)^{-n / 2-1 / 2 \min \{|\alpha|, \ell-2-|\alpha|\}} \int_{t / 2}^{t}(1+(t-s))^{n / 2(1 / p-1)-1 / 2} d s
$$$$
+C(|\alpha|) E^{2} \int_{0}^{t} e^{-b(t-s)}(1+s)^{n / 2(1 / p-1)-1 / 2 \min \{|\alpha|, \ell-3-|\alpha|\}-1 / 2} d s,
$$

$$
\leq C(|\alpha|) E^{2} L(t)(1+t)^{n / 2(1 / p-1)-1 / 2 \min \{|\alpha|, \ell-3-|\alpha|\}+((n-1) / 4)(2 / p-1)-1 / 2} \text {. }
$$

In the case $|\alpha|=0$, we can replace $\int_{t / 2}^{t}\left\|D_{x}^{2} \tilde{G} * D_{x}^{\beta} Q_{2}\right\|_{p}$ with $\int_{t / 2}^{t}\left\|D_{x} \tilde{G} * D_{x}^{\alpha} Q_{2}\right\|_{p}$ in the first line of $(8.23)$ and obtain the same result.

From (8.18), (8.19) and (8.23), we obtain (8.12) and

$$
\begin{gathered}
\left\|D_{x}^{\alpha}\left(u-G * u_{0}\right)\right\|_{p} \\
\leq C(|\alpha|) E^{2} L(t)(1+t)^{n / 2(1 / p-1)-1 / 2 \min \{|\alpha|, \ell-3-|\alpha|\}+((n-1) / 4)(2 / p-1)-1 / 2} .
\end{gathered}
$$


Equations (8.16) and (8.17) give

$$
\begin{gathered}
\left\|D_{x}^{\alpha}(G-\tilde{G}) * u_{0}\right\|_{p} \\
\leq C(|\alpha|, \sigma) E(1+t)^{n / 2(1 / p-1)-|\alpha| / 2+(n / 4)(2 / p-1)-1 / 2+\sigma} .
\end{gathered}
$$

Triangulating with (8.24), we find that

$$
\begin{gathered}
\left\|D_{x}^{\alpha}\left(u-\tilde{G} * u_{0}\right)\right\|_{p} \\
\leq C(|\alpha|, \sigma) E(1+t)^{n / 2(1 / p-1)-1 / 2 \min \{|\alpha|, \ell-3-|\alpha|\}+(n / 4)(2 / p-1)-1 / 2+\sigma},
\end{gathered}
$$

as well.

Similar analysis applied to the artificial viscosity system (1.7), gives

$$
\begin{gathered}
\left\|D_{x}^{\alpha}\left(\tilde{u}-\tilde{G} * u_{0}\right)\right\|_{p} \\
\leq C(|\alpha|) E^{2} L(t)(1+t)^{n / 2(1 / p-1)-1 / 2 \min \{|\alpha|, \ell-3-|\alpha|\}+((n-1) / 4)(2 / p-1)-1 / 2},
\end{gathered}
$$

just as in (8.24). Triangulating with (8.26), we obtain

$$
\begin{gathered}
\left\|D_{x}^{\alpha}(u-\tilde{u})\right\|_{p} \\
\leq C(|\alpha|, \sigma) E(1+t)^{n / 2(1 / p-1)-1 / 2 \min \{|\alpha|, \ell-3-|\alpha|\}+(n / 4)(2 / p-1)-1 / 2+\sigma},
\end{gathered}
$$

completing the proof of (8.13).

Finally, the proof of Theorem 6.8 shows that

$$
\begin{aligned}
\left\|D_{x}^{\alpha}\left(\tilde{G} * u_{0}-\tilde{G} U\right)\right\|_{p} & \leq\left\|D_{x}^{\alpha} D_{x} \tilde{G}\right\|_{p}\left\|x u_{0}\right\|_{1}, \\
& \leq C(1+t)^{n / 2(1 / p-1)-|\alpha| / 2+((n-1) / 4)(2 / p-1)-1 / 2}\left\|x u_{0}\right\|_{1} .
\end{aligned}
$$

Triangulating with (8.26) gives (8.14), completing the proof in the case $3 \leq \ell$, $|\alpha| \leq \ell-2$. The case that $|\alpha|=\ell-1$ or $\ell \leq 2$ follows similarly.

Theorem 8.2 shows that the convected diffusion wave $\tilde{G}=W * G^{\tilde{B}}$ dominates the asymptotic behavior of $u$, also in $L^{p}, p<2$.

\section{REFERENCES}

[1] S. Godunov, An interesting class of quasilinear systems, Soviet Math. 2 (1961), 947-949.

[2] David Hoff, Global well-posedness of the Cauchy problem for the Navier-Stokes equations of nonisentropic flow with discontinuous initial data, J. of Differential Equations 95, No.1 (1992), 33-73.

[3] David HofF, Global solutions of the Navier-Stokes equations for multidimensional, compressible flow with discontinuous initial data J. Diff. Eqns, (to appear). 
[4] David Hoff, Strong convergence to global solutions for compressible, viscous, multidimensional flow, with polytropic equations of state and discontinuous initial data, Archive Rational Mech. Anal. (to appear).

[5] David Hoff and Joel Smoller, Global existence for systems of parabolic conservation laws in several space variables, J. Diff. Eqns. 68 (1987), 210-220.

[6] David Hoff and Kevin Zumbrun, Pointwise decay estimates for mulidimensional diffusion waves, (in preparation).

[7] Ya. KANEL, On a model system of equations of one-dimensional gas motion, Diff. Eqns. 4 (1968), 374-380.

[8] S. KaWASHIMA, Systems of a hyperbolic-parabolic composite type, with applications to the equations of magnetohydrodynamics, Ph. D. Thesis, Kyoto University, 1983.

[9] S. KaWASHima, Large-time behavior of solutions to hyperbolic-parabolic systems of conservation laws and applications, Proc. Roy. Soc. Edinburgh 106A (1987), 169-194.

[10] P. LAX AND K.O. Friedrichs, Systems of conservation laws with a convex extension, Proc. Nat. Acad. Sci. USA 68, (1971), 1686-1688.

[11] P.L. Lions, Existence globale de solutions pour les équations de Navier-Stokes compressibles isentropiques, C.R. Acad. Sci. Paris 316 (1993), 1335-1340.

[12] TAI-PING LiU, Nonlinear stability of shock waves for viscous conservation laws, Memoirs AMS 56, no. 328 (1985).

[13] TAI-PING LiU, Pointwise convergence to shock waves for the system of viscous conservation laws, (Preprint).

[14] Tai-Ping Liu and Kevin Zumbrun, Nonlinear stability of an undercompressive shock of complex Burgers equation Comm. Math. Phys, (to appear).

[15] Tai-Ping LiU and Zhouping Xin, to appear.

[16] TAI-PING LIU AND Y. ZENG, Large time behavior of solutions of general quasilinear hyperbolic-parabolic systems of conservation laws, (Preprint) (1994).

[17] Akitaka Matsumura and TakaAki Nishida, Initial boundary value problems for the equations of motion of compressible viscous and heat conductive fluids, Comm. Math. Phys. 89 (1983).

[18] Elias M. Stein, Singular Integrals and Differentiability lllllllProperties of Functions, Princeton Univ. Press, Princeton, N.J., 1970.

[19] Walter Strauss, Nonlinear Wave Equations, CBMS ser. 91 (1989).

[20] Anders Szepessy And Zhouping XIN, Nonlinear stability of viscous shock waves, Arch. Rat. Mech. Anal. 122 no. 1 (1993), 53-103.

[21] Anders Szepessy And Kevin Zumbrun, Stability of rarefaction waves in viscous media, (Preprint) (1994)).

[22] Y. ZENG, $L^{1}$ asymptotic behavior of compressible, isentropic, viscous $1 d$ flow Comm. Pure Appl. Math, (to appear).

Research of the first author was supported in part by the NSF under Grant No. DMS-9322274. Research of the second author was supported in part by the ONR under Grant No. N0001494-1-0456 and by the NSF under Grant No. DMS-9107990.

Department of Mathematics

Indiana University

Bloomington, Indiana 47405

Received: April 10th, 1995. 